\title{
Modeling of Strain Induced Pockels Effect in Silicon Photonic Waveguides
}

by

Seyed Alireza Aleali, B.Sc., M.B.A.

A thesis submitted to the Faculty of Graduate and Postdoctoral Affairs in partial fulfillment of the requirements for the degree of

Master of Applied Science

in

Electrical and Computer Engineering

Ottawa-Carleton Institute for Electrical and Computer Engineering Department of Electronics

Carleton University

Ottawa, Canada

(C) 2014, Seyed Alireza Aleali 


\section{Abstract}

Linear phase modulation is a critical characteristic of state-of-the-art modulators in coherent optical communication systems. Currently the majority of silicon modulators being developed are based on the plasma dispersion effect since silicon's centrosymmetric crystal structure prohibits the Pockels (or linear electro-optic) effect. The plasma dispersion effect is nonlinear with speed limited by charge mobility. Recently it was shown that by applying an anisotropic strain to silicon waveguides, the symmetry of the crystal structure can be broken, hence, artificially inducing the Pockels effect.

In this dissertation we investigate the strain induced Pockels effect in silicon waveguides. We introduce a Figure of Merit (FOM) that characterizes the Pockels effect in various silicon waveguides and show the proposed FOM has an excellent correlation with the experimentally obtained second order nonlinearity $\chi^{(2)}$. A six-fold improvement of FOM can be achieved by optimizing the waveguide cross-section and tuning the mechanical properties of the straining layer. 


\section{Dedicated to my parents $\mathcal{M}$ ery and $\mathcal{M}$ ajid for their love, endless support, and encouragement; \\ And to my late uncle Saeed}

for inspiration to pursue $\mathcal{E}$ ngineering as my career. 


\section{Acknowledgements}

My sincerest appreciation goes to my professor, Dr. Winnie Ye, for her patience, advice, and guidance throughout my research. She has tremendously contributed to my professional growth. Heartfelt thanks goes to Dr. Jens Schmid for his constant help, concern, and support throughout my studies. I am also deeply grateful to Dr. Dan-Xia Xu and Dr. Pavel Cheben for their guidance and numerous discussions we had on various aspects of this project. The extensive expertise of all my advisors in the field of silicon photonics has been instrumental in completion of this thesis and without them this work would have never been completed.

I would also like to thank my colleagues and fellow students at Carleton University, especially Marc Ibrahim, Yule Xiong, Bryce Dorin and Ryan Veenkamp. They have helped me learn and grow both personally and professionally. All the fun we had together during our graduate studies helped me go through the tough times and celebrate the good times.

At last but not least, I thank my wife Oksana for her infinite support, love, and cheerful encouragement. 


\section{Table of Contents}

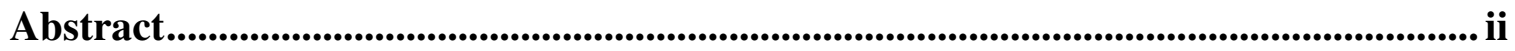

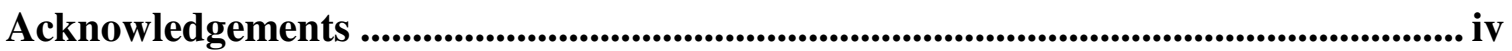

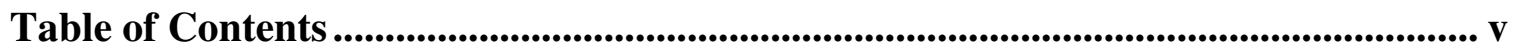

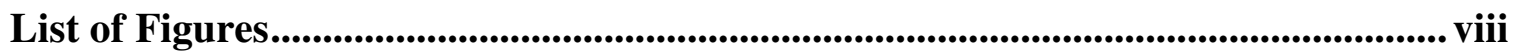

List of Tables ......................................................................................................................................... xii

1 Chapter: Introduction and Thesis Organization ............................................................ 1

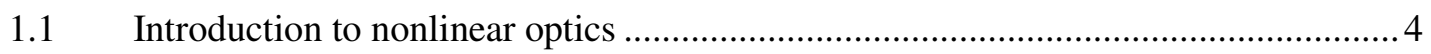

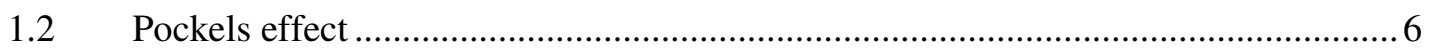

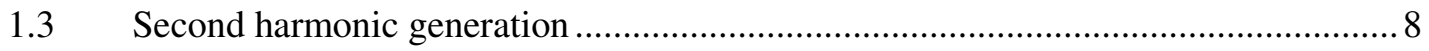

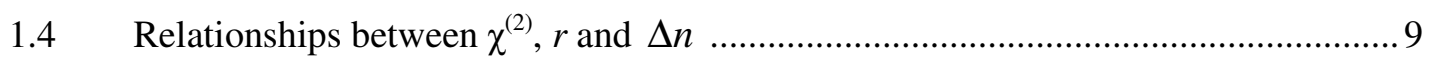

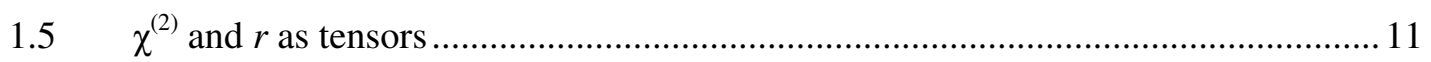

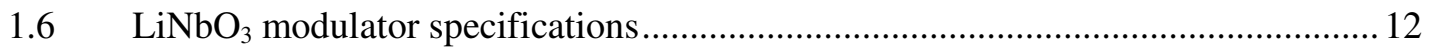

2 Chapter: Literature Review.......................................................................................... 15

2.1 Second harmonic generation at silicon surfaces ............................................... 15

2.2 Second harmonic generation in silicon waveguides.......................................... 17

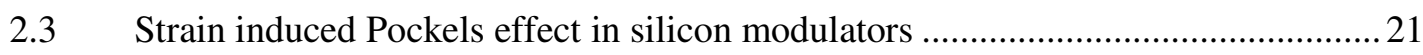

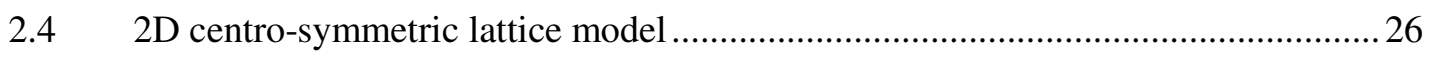

3 Chapter: Strain Simulations ............................................................................................. 28

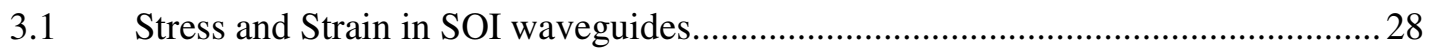

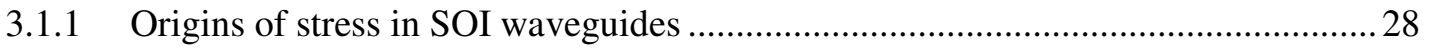

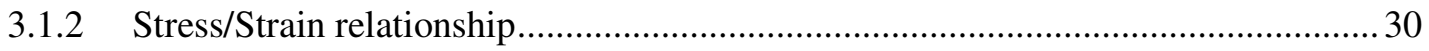

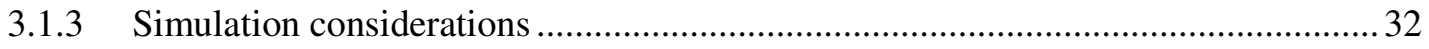

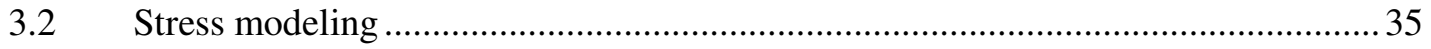

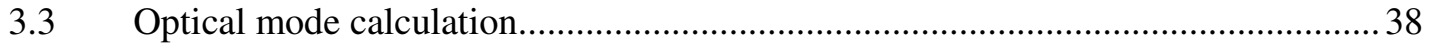




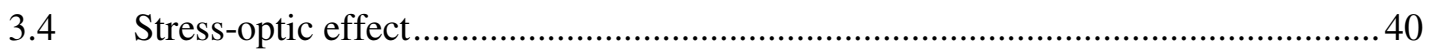

4 Chapter: Stress/Strain Characterization Using Figure of Merit............................ 41

4.1 Analysis of the strain induced Pockels effect in silicon waveguides ....................... 41

4.1.1 Micro-scale channel waveguide .................................................................... 41

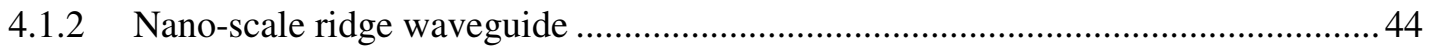

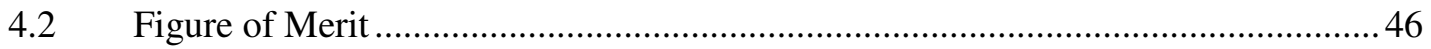

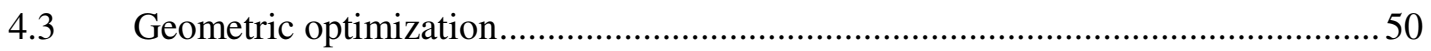

4.3.1 Optimization of nano-scale waveguide with gaps in cladding ...............................50

4.3.2 Optimization of nano-scale waveguide with uniform cladding ..............................53

4.3.3 Optimization of strain induced effects in nano scale slanted rib waveguide ...........57

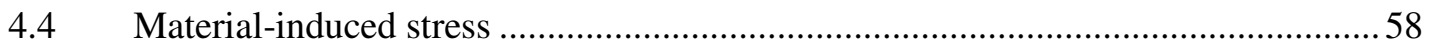

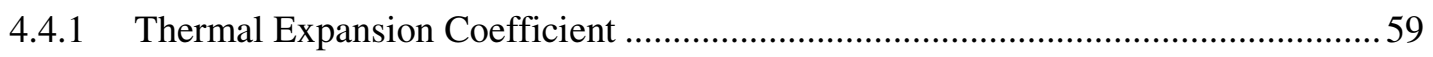

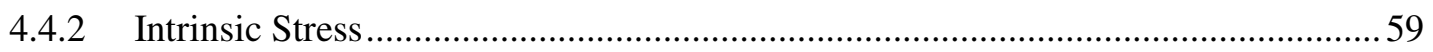

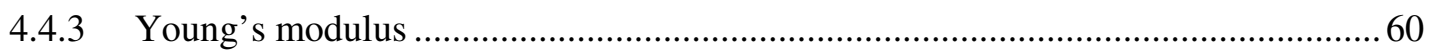

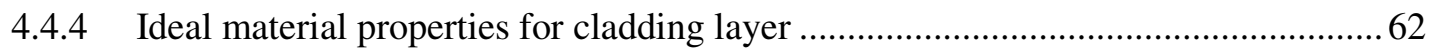

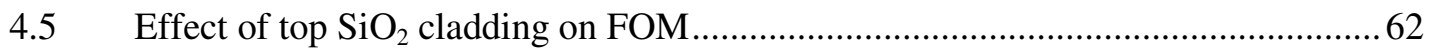

4.6 Position of the stress relief gap in straining layer ..............................................65

5 Chapter: Enhancement of Pockels Effect in Silicon Waveguides ........................ 68

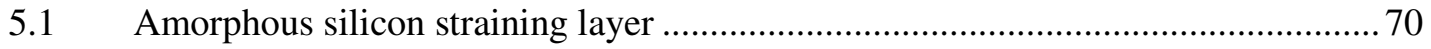

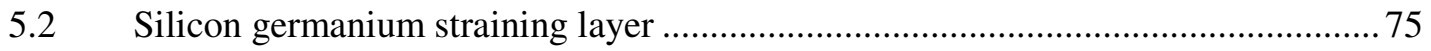

6 Chapter: Conclusion and Future Work................................................................ 80

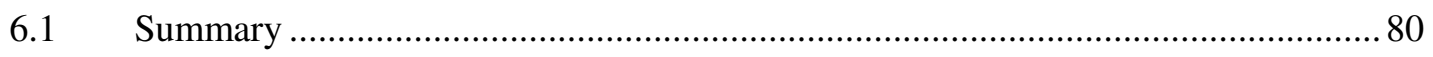

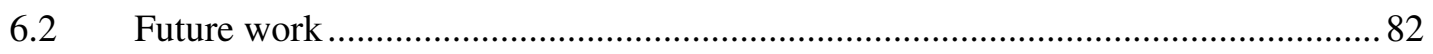

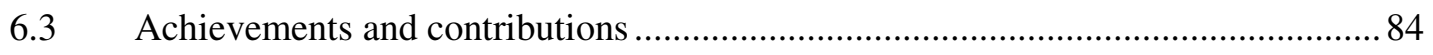

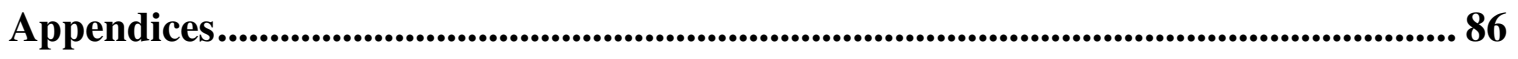


Appendix A Geometrical parameters for waveguides used in Figure 4.3 plot that correlates

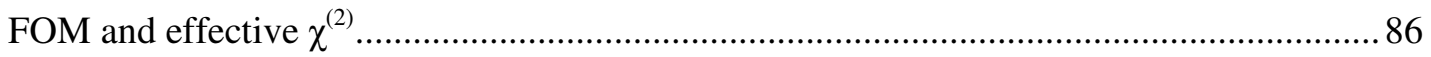

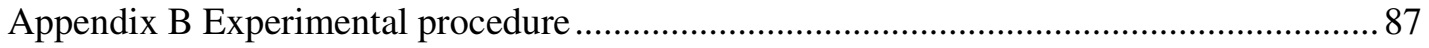

Appendix C Derivation of electric field due to 2D centro-symmetric lattice .......................90

Appendix D MATLAB code for the Comsol ${ }^{\circledR}$ program ….................................................. 92

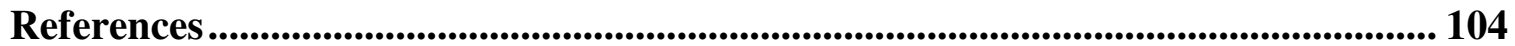




\section{List of Figures}

Figure 1.1 Schematic of a (a) centro-symmetric and (b) non-centro-symmetric structure $[20]$

Figure 1.2 An electric field applied to an electro-optic material changes its refractive index and therefore phase of the propagating light [25].

Figure 1.3 Dependence of refractive index on electric field: (a) Pockels medium; (b) Kerr medium [25]. 7

Figure 1.4 Second harmonic generation results from absorption of two photons at

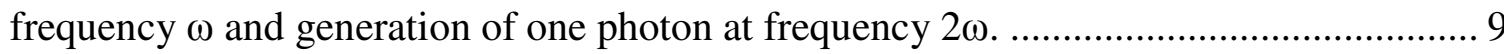
Figure 1.5 Schematic of a Mach-Zehnder Interferometer [26]...................................... 13

Figure 2.1 Second harmonic beams reflected from silicon surface................................. 16 Figure 2.2 Linear dependence of second order non-linearity coefficients $\mathrm{a}_{\mathrm{pp}}$ and $\mathrm{c}_{\mathrm{pp}}$ on

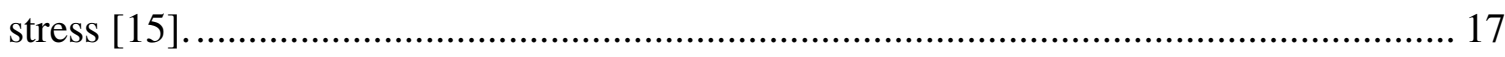

Figure 2.3 Strained silicon waveguides used to produce second harmonic signals......... 18 Figure 2.4 Schematic of cells used for ab-initio simulations to calculate strain induced $\chi^{(2)}$

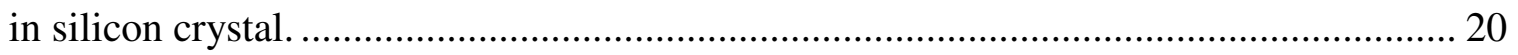

Figure 2.5 Schematic of effect of straining layer on a silicon waveguide....................... 21

Figure 2.6 Schematic of (a) the Mach-Zehnder Interferometer used to demonstrate the Pockels effect and (b) Photonic Crystal Wire (PCW) used to ehance $\chi^{(2)}[7] \ldots \ldots \ldots \ldots \ldots . . . .22$ Figure $2.7 \chi_{e n h}^{(2)}$ in silicon waveguide as function of wavelength before and after

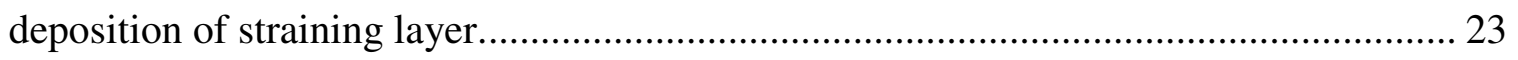

Figure 2.8 Cross section of a rib waveguide with $\mathrm{Si}_{3} \mathrm{~N}_{4}$ straining layer......................... 24

Figure 2.9 First fully integrated strain induced Pockels effect modulator....................... 25 
Figure 2.10 Schematic of a 2D centro-symmetric silicon lattice structure under

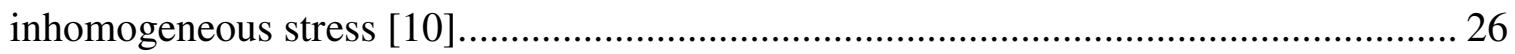

Figure 3.1 Typical cross section of SOI platform with a straining layer....................... 28

Figure 3.2 The cross section and geometrical parameters of a rib waveguide with silicon nitride upper cladding layer on SOI platform................................................... 29

Figure 3.3 Intrinsic and total stress of a $\mathrm{Si}_{3} \mathrm{~N}_{4}$ thin film as functions of low frequency

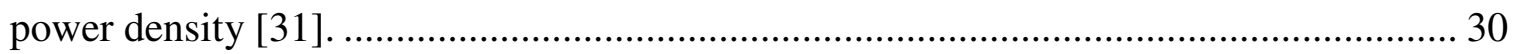

Figure 3.4 Structure modeled for mechanical stress-strain calculations........................ 34

Figure 3.5 Stress distribution along $x$ at the mid-section of straining layer. .................. 35

Figure 3.6 Stress component $\left(\sigma_{\mathrm{xx}}\right)$ distribution in SOI1 and SOI2 type slabs. ............... 36

Figure 3.7 Stress and strain profiles for strained $\mu \mathrm{m}$ scale channel silicon waveguides. 38 Figure 3.8 Optical calculation windows for the $\mu \mathrm{m}$ scale channel waveguides.

Figure 4.1 Strain-induced effects for a $2.3 \mu \mathrm{m}$ wide channel waveguide in $2 \mu \mathrm{m}$ thick SOI. 43

Figure 4.2 Strain induced effects for a $500 \mathrm{~nm}$ wide ridge waveguide in $220 \mathrm{~nm}$ SOI. The $350 \mathrm{~nm}$ thick $\mathrm{Si}_{3} \mathrm{~N}_{4}$ layer has gaps near waveguide sidewalls. Film stress $\sigma_{\text {film }}=+1 \mathrm{GPa}$.

Figure 4.3 Correlation between experimental second order nonlinearity $\chi^{(2)}$ values reported in [13], [14] and the calculated Figure of Merit (FOM).

Figure 4.4 Figure of merit and effective $\chi^{(2)}$ as functions of waveguide width. 50 Figure 4.5 Optimization of strain induced effects in a nano scale rib waveguide with cracks in cladding layer. 52 
Figure 4.6 Strain induced effects for a $500 \mathrm{~nm}$ wide ridge waveguide in $220 \mathrm{~nm}$ SOI. The $350 \mathrm{~nm}$ thick $\mathrm{Si}_{3} \mathrm{~N}_{4}$ layer covers waveguide sidewalls uniformly. Film stress $\sigma_{\text {film }}=+1$

GPa. 54

Figure 4.7 Optimization of strain induced effects in a nano-scale rib waveguide where cladding layer covers the waveguide sidewalls uniformly. 56

Figure 4.8 Stress induced effect for a $450 \mathrm{~nm}$ wide slanted channel waveguide. 58 Figure 4.9 Linear relationship between thermal expansion coefficient (TEC) of $\mathrm{Si}_{3} \mathrm{~N}_{4}$ and (a) total stress, and (b) FOM. 59

Figure 4.10 Linear relationship between intrinsic stress of $\mathrm{Si}_{3} \mathrm{~N}_{4}$ and FOM. 60 Figure 4.11 Effect of Young's modulus on (a) total stress and (b) FOM when thermal expansion coefficient (TEC) of silicon nitride is $3.6 \mu \mathrm{K}^{-1}$. 61 Figure 4.12 Strain induced effects in silicon rib waveguide with $\mathrm{Si}_{3} \mathrm{~N}_{4}$ straining layer and $850 \mathrm{~nm} \mathrm{SiO}_{2}$ layer. 63

Figure 4.13 Optimization of strain induced effects in silicon rib waveguide with $\mathrm{Si}_{3} \mathrm{~N}_{4}$

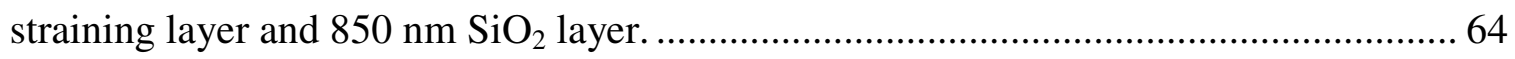
Figure 4.14 Influence of gap on distribution of strain gradient and FOM...................... 66 Figure 5.1 Comparison of strain-induced effects for waveguides with $\mathrm{Si}_{3} \mathrm{~N}_{4}$ and a-Si straining layers 69 Figure 5.2 Normalized optical mode distribution in waveguides with a-Si straining layer. Straining layer thickness is 50, 125 and $300 \mathrm{~nm}$ for (a), (b) and (c), respectively. 72 Figure 5.3 Optimization of strain induced effects for a nano scale channel waveguide with a-Straining layer. 73 
Figure 5.4 Figure of Merit as a function of width of the silicon core for $E_{\mathrm{a}-\mathrm{Si}}=40$ and 63 GPa.

Figure 5.5 (a) Total stress in SiGe as a function of Ge content. (b) Bandgap energy of

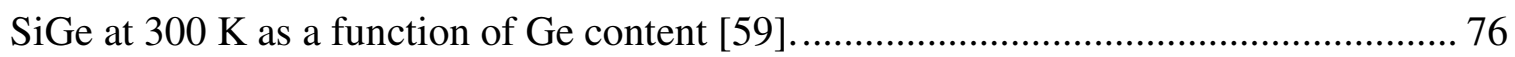

Figure 5.6 Strain induced effects for silicon waveguide with SiGe straining layer. ........ 77 Figure 5.7 FOM as a function of straining layer thickness for filmstress of $\sigma_{\text {film }}=-1 \mathrm{GPa}$ and $-2 \mathrm{GPa}$, corresponding to $p=0.85$ and 0.7 , respectively................................... 78

Figure 5.8 Strain induced effects for a nano scale slot channel waveguide. .................. 79 Figure 6.1 Strain induced effect for waveguide structures with horizontal asymmetric

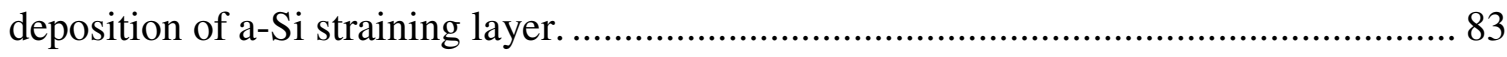




\section{List of Tables}

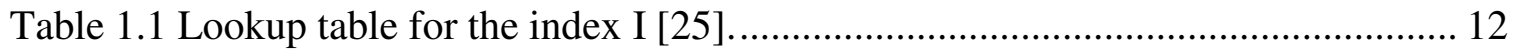

Table 3.1: Physical properties for material typically used for various layers of strained

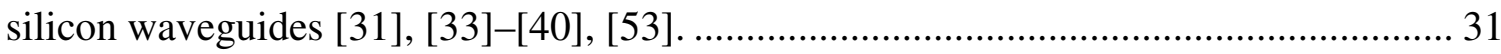

Table A. 1 Geometrical parameters for waveguides used in Figure 4.3 plot that correlates calculated FOM and experimentally measured effective $\chi^{(2)}$ 86 


\section{Chapter: Introduction and Thesis Organization}

Silicon photonics is a promising technology poised to revolutionize datacom, telecom, spectroscopy and biosensing applications by providing low priced and small footprint devices. The research in this field has grown rapidly in the past decade. A major motivation behind research in silicon photonics is cost reduction and high yields possible when leveraging Complementary Metal-Oxide Semiconductor (CMOS) fabrication facilities that have been extensively developed for electronics chips. Silicon is transparent in the c-band and thus can be used for telecom applications. The high refractive index of silicon allows for devices with very small footprints and tight waveguide bends in Photonic Integrated Circuits (PIC). Various components needed for generation, transport and detection of data over optical fiber have been developed on a silicon platform to perform functions such as lasing, modulating, wavelength switching and coupling [1][4].

Optical modulation is the process of encoding data in a stream of light. Various research groups around the world have been working on silicon optical modulators. The majority of the existing work is on plasma dispersion effect which utilizes charge carriers inside doped silicon to alter the refractive index of the silicon waveguide and thus inducing a

phase shift in the propagating light [2]. Typically two phase shifters are placed in a Mach-Zehnder Interferometer (MZI) configuration to form a basic On-Off-Keying modulation scheme.

Although recently plasma dispersion based silicon modulators have achieved high speeds and small foot prints there are various limitations in using the plasma dispersion effect. Firstly, the phase shift due to change in carrier concentration inside waveguides is a 
nonlinear function of the applied voltage that also causes chirp [5]. The two arms of a MZI cannot produce phase shifts that exactly interfere since exact doping of each arm is slightly different, although they have undergone the same fabrication process. The nonlinear phase shift in modulators is especially troublesome for long-haul applications where advanced modulation schemes are required in order to increase spectral efficiency. Advanced modulation schemes such as Differential Phase Shift Keying (DPSK) and Quadrature Amplitude Modulation (QAM) multiply symbol rates by two or more (when compared to simple On-Off-Keying) by modulating the phase as well as the amplitude.

Secondly, the plasma dispersion effect is limited by speed or mobility of the free-carriers that change the refractive index. In addition, typically a high a-c current is needed to run through the silicon to move the free-carriers [6].

In contrast, state-of-the-art modulators used in telecommunications industry are based on the linear electro-optics (EO) effect, also known as the Pockels effect. The linear EO effect is an electric field induced effect that does not require movement of free-carriers inside the waveguide. It is the response of non-centro-symmetric crystals to an applied electric field. The response is in form of a change in polarization at the atomic scale. Therefore, the Pockels effect is a faster and more power efficient process compared to the plasma dispersion effect.

Unfortunately silicon does not exhibit a Pockels effect because it has a diamond cubical crystal with inversion symmetry. This prohibits the existence of the linear electro-optic effect, as explained later in this chapter. However, in 2006 two research groups demonstrated that the central symmetry of the silicon crystal can be broken by deposition of highly strained materials on SOI waveguides [7], [8]. In 2012 the first fully integrated 
silicon Pockels effect modulator was reported [9]. The current research in the literature has been focused on proof of concept devices without trying to optimize the strain induced Pockels effect in silicon.

Theoretically, the exact physical relationship between the applied strain and the induced Pockels effects in silicon waveguides is still being debated [10]-[12]. Experimentally, however, most results indicate a direct relationship between strain gradient and the induced Pockels effect [13]-[15].

The induced Pockels effect in silicon is considered to be a significant discovery that might lead to a new class of silicon optical modulators that can compete in performance with state of the art Lithium Niobate modulators. Motivated by this fact and recent developments, in this thesis we investigate strain induced Pockels in silicon and propose a relatively simple approach to optimize the effect.

In the remainder of this chapter we review fundamentals of nonlinear optics as it relates to the Pockels effect and the Second Harmonic Generation to provide the background necessary for understanding of the thesis. Chapter 2 presents a review of the recent work on second harmonic generation in silicon substrates and waveguides as well as the Pockels effect in silicon waveguides.

Chapter 3 covers the details of the numerical techniques and software used to conduct investigation of the strain distribution and optical modes in waveguides. In chapter 4 we analyze the mechanical and optical properties of the waveguide structures reported in the literature. We then introduce a Figure of Merit (FOM) to quantitatively compare the strength of the Pockels effect in various strained silicon waveguides. Using the 
introduced FOM optimization of a nano-wire rib waveguides with $\mathrm{Si}_{3} \mathrm{~N}_{4}$ cladding/straining layer is presented.

In Chapter 5 the limitations of the typical strained silicon waveguides for the Pockels effect modulators which utilize $\mathrm{Si}_{3} \mathrm{~N}_{4}$ as cladding/straining layer are discussed. Then, a new device structure is proposed that circumvents those limitations by using a high refractive index straining material such as $\mathrm{SiGe}, \mathrm{SiC}$, and $\alpha$-Si. The new design provides for a substantial enhancement of the Pockels effective in silicon waveguides by engineering of the optical mode.

In chapter 6 a summary of the key findings is presented. A discussion of the future direction of the research is also provided.

\subsection{Introduction to nonlinear optics}

Nonlinear optics is the study of effect of light and electric fields on optical properties of a material. In conventional (linear) optics, response of material to a time varying applied electric field $\vec{E}(t)$ can be described by linear polarization:

$$
\vec{P}(t)=\varepsilon_{0} \chi^{(1)} \vec{E}(t)
$$

where $\varepsilon_{0}$ is permittivity of free space and $\chi^{(1)}$ is the constant of proportionality known as the linear susceptibility. However, in asymmetrical crystals polarization induced by strong optical fields and by dc electric fields cannot be approximated as a linear function of the applied field. In these crystals the response of atoms to dc electric fields and optical fields leads to displacement of electrons from the equilibrium position. The displacement becomes so large the electronic potentials of the neighboring atoms must be taken into consideration in order to describe the change in the polarization accurately. This large displacement leads to additional terms in polarization that depend on the 
second, third or higher power of the incident field [16]. Time dependent nonlinear polarization $\vec{P}^{\mathrm{NL}}(t)$ can be described by a power series in the field strength $\vec{E}$ as:

$$
\vec{P}^{\mathrm{NL}}(t) \equiv \vec{P}^{(2)}+\vec{P}^{(3)}+\ldots=\varepsilon_{0}\left[\chi^{(2)} \vec{E}^{2}(t)+\chi^{(3)} \vec{E}^{3}(t)+\ldots\right]
$$

where $\chi^{(2)}$, and $\chi^{(3)}$ are second and third-order susceptibilities. The terms $\vec{P}^{(2)}=\varepsilon_{0} \chi^{(2)} \vec{E}^{2}(t)$ and $\vec{P}^{(3)}=\varepsilon_{0} \chi^{(3)} \vec{E}^{3}(t)$ are referred to as second-order and third-order nonlinear polarization since they change polarization nonlinearly. Higher order terms are often ignored since their effect is negligible in practice.

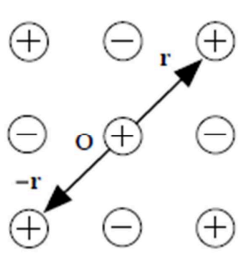

(a)

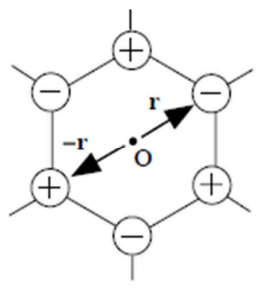

(b)

Figure 1.1 Schematic of a (a) centro-symmetric and (b) non-centro-symmetric structure [20].

Crystalline group IV materials such as such as silicon ( $\mathrm{Si})$, germanium $(\mathrm{Ge})$ and carbon (C) have diamond lattice structure which is centro-symmetric. Centro-symmetric crystals have inversion symmetry with a central point through which every atom can be reflected into an identical atom [17] in other words for every point $(\mathrm{x}, \mathrm{y}, \mathrm{z})$ in the unit cell there is an indistinguishable point (-x, -y, -z) [18], as illustrated in Figure 1.1.

Crystals that have inversion symmetry reverse the polarization when the direction of applied electric field is changed; that is in equation (1.2) if $\vec{E}(t)$ becomes $-\vec{E}(t)$ the polarization $\vec{P}(t)$ becomes $-\vec{P}(t)$. The only case that allows such change is when $\chi^{(2)}=0$. Therefore presence of inversion symmetry restricts the second order nonlinearity $\chi^{(2)}$ to be zero. However, central symmetry is naturally broken at the interface of crystal and 
induces a weak $\chi^{(2)}$ near the surface [19]. In waveguides the symmetry can also be broken by application of a straining layer deposited on the top, that can lead to presence of a strong $\chi^{(2)}$ in silicon waveguides.

\subsection{Pockels effect}

In the Pockels effect the second order nonlinear polarization is dependent on an applied dc electric field:

$$
\vec{P}^{(2)}=\varepsilon_{0} \chi^{(2)} \vec{E}_{O p t} \vec{E}_{d c}(t)
$$

where $\vec{E}_{O p t}$ is the electric field at optical frequency and $\vec{E}_{d c}$ is the dc (or modulation) electric field. Figure 1.2 shows a schematic demonstrating the electro-optic effect.

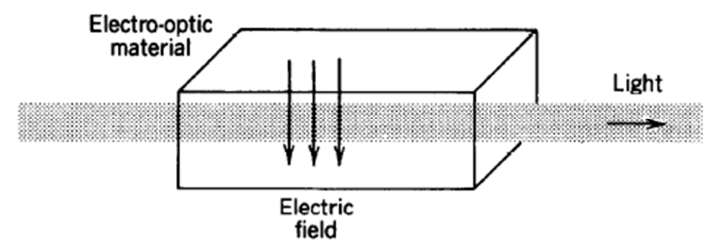

Figure 1.2 An electric field applied to an electro-optic material changes its refractive index and therefore phase of the propagating light [25].

Although the linear electro-optic effect can be described in terms of second-order nonlinear susceptibility $\chi^{(2)}$, historically a very different mathematical formalism has been used [16]. The Pockels effect results in a linear change of refractive index $n$ as function of an applied electric field:

$$
n=n(E)
$$

The dependence of refractive index on electric field can be expanded as a Taylor series.

$$
n=n_{0}+\Delta n=n_{0}+a_{1} E+\frac{1}{2} a_{2} E^{2}+\ldots
$$


where $n_{0}$ is the constant part of the refractive index, and coefficients $a_{1}$ and $a_{2}$ are defined as:

$$
\begin{gathered}
a_{1}=\left.\frac{d n}{d E}\right|_{E=0} \\
a_{2}=\left.\frac{d^{2} n}{d E^{2}}\right|_{E=0}
\end{gathered}
$$

It is customary to define new coefficients $r=-2 a_{1} / n_{0}^{3}$ and $s=-a_{2} / n_{0}^{3}$ so that:

$$
n=n_{0}-\frac{1}{2} r n_{0}^{3} E-\frac{1}{2} s n_{0}^{3} E^{2}+\ldots
$$

$r$ and $s$ are often called Pockels and Kerr coefficients, respectively [20]. Typical values for $r$ lie in the range of 1 to $100 \mathrm{pm} / \mathrm{V}$ and for $s$ are around $10^{-18}$ to $10^{-14} \mathrm{~m}^{2} / \mathrm{V}^{2}$. The change in $n$ due to $E$ is referred to as Pockels effect while the change in $n$ due to $E^{2}$ is called the Kerr effect. All materials display Kerr effect however only crystals with no inversion symmetry display the Pockels effect. Figure 1.3 shows how refractive index changes in Pockels medium and Kerr medium when an electric field applied. Note the asymmetrical nature of the change in the Pockels medium.

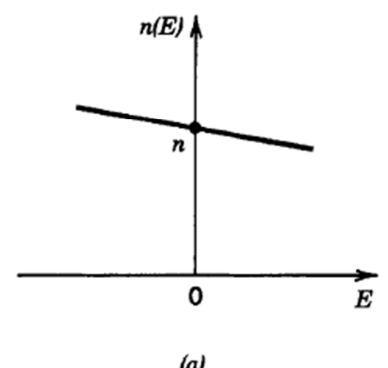

(a)

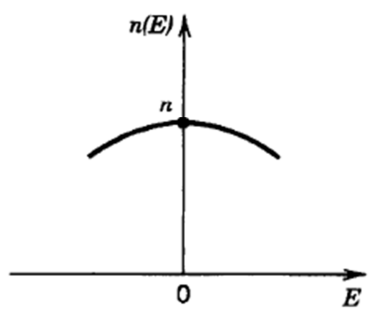

(b)

Figure 1.3 Dependence of refractive index on electric field: (a) Pockels medium; (b) Kerr medium [25]. 


\subsection{Second harmonic generation}

Closely related to the Pockels effect is a phenomenon called second harmonic generation (SHG). Both effects are caused by the same nonlinear coefficient, namely $\chi^{(2)}$, with the main difference being the frequency of the applied field. In the Pockels effect the $E$ field is at $\mathrm{MHz}$ or $\mathrm{GHz}$ ranges which are usually considered dc frequencies in photonics. In SHG, the $E$ field is at optical frequency range (hundreds of $\mathrm{THz}$ ). The polarization changes with respect to the frequency of the applied field. Studying results developed from SHG experiments in silicon is critical for understanding the nature of the strain induced Pockels effect in silicon waveguides.

The second harmonic generation phenomena, which was discovered by Franken et al. in 1961 [21], is a technologically important application of nonlinear optics. One of the main applications of SHG is material diagnosis by probing surfaces of the material. SHG signals have strong dependence on material symmetry therefore any change that affects the symmetry of the material can be investigated. In fact, SHG signals reflected from centro-symmetric semiconductor (such as $\mathrm{Si}$ and $\mathrm{Ge}$ ) surfaces have been used as a tool for determining sample orientation and monitoring surface changes such as melting, defect generation and amorphisation [22]. These changes modify the intensity of the SH and its dependence on the crystal orientation [19].

Let us consider a monochromatic laser beam that is incident on a crystal. The beam has an electric field in the form of:

$$
\vec{E}(t)=E_{0} \sin (\omega \mathrm{t})
$$

where $\omega$ is frequency and $E_{0}$ is the constant field. According to equation (1.2) the second-order polarization that is created in such a crystal is given as $\vec{P}^{2}(t)$ : 


$$
\vec{P}^{(2)}(\mathrm{t})=\varepsilon_{0} \chi^{(2)} E_{0}{ }^{2}\left[\frac{1}{2}-\frac{1}{2} \cos (2 \omega \mathrm{t})\right]
$$

The first term in the bracket is independent of the frequency and leads to a static (i.e. dc) electric field. The second term in the bracket has dependence on the frequency $2 \omega$. This is the term that leads to second harmonic generation in material under intense incident light.

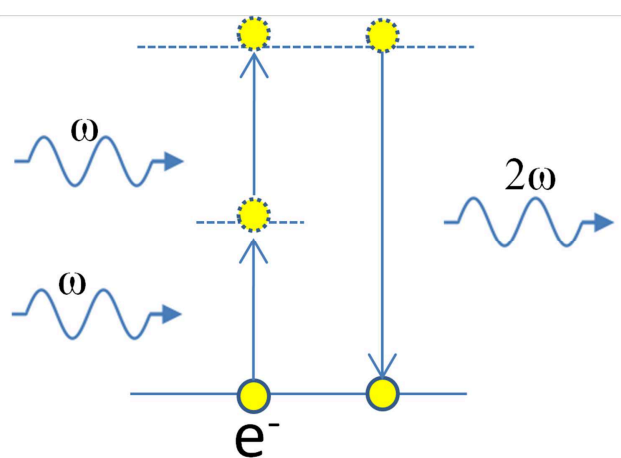

Figure 1.4 Second harmonic generation results from absorption of two photons at frequency $\omega$ and generation of one photon at frequency $2 \omega$.

Phenomenological explanation can be given as such: when the intense light with a frequency $\omega$ incidents on a crystal, two photons are simultaneously annihilated to excite an electron to a higher virtual energy level. The excited electron then relaxes to the ground state and emits a photon with a frequency of $2 \omega$. This process is schematically demonstrated in Figure 1.4. It must be noted this process is not limited to two photons with exact same frequencies. Additional processes leading to sum-frequency and difference-frequency generation are also possible [23], that are outside scope of this work.

\subsection{Relationships between $\chi^{(2)}, r$ and $\Delta n$}


In this section we will discuss the relationship between second order nonlinearity $\chi^{(2)}, r$ and change in the refractive index $\Delta n$. In nonmagnetic materials the refractive index is related to the susceptibility by:

$$
n_{0}=\sqrt{1+\chi^{(1)}}
$$

More generally we can write:

$$
n=\sqrt{1+\chi_{e f f}}
$$

where $\chi_{\text {eff }}$ is the effective susceptibility that includes higher order nonlinearities. In case of the second-order nonlinearity [24]:

$$
n=\sqrt{1+\chi^{(1)}+2 \chi^{(2)} E}
$$

Then,

$$
n=\sqrt{\left(1+\chi^{(1)}\right)\left(1+\frac{2 \chi^{(2)} E}{1+\chi^{(1)}}\right)}
$$

Using (1.11) we can write:

$$
n=n_{0} \sqrt{\left(1+\frac{2 \chi^{(2)} E}{n_{0}^{2}}\right)}
$$

Since, $\frac{\chi^{(2)} E}{1+\chi^{(1)}}<<1$,

$$
n \simeq n_{0}\left[1+\frac{1}{2}\left(\frac{2 \chi^{(2)} E}{n_{0}{ }^{2}}\right)\right]
$$

Using (1.5) and (1.16),

$$
n_{0}\left[1+\frac{\chi^{(2)} E}{n_{0}{ }^{2}}\right]=n_{0}+\Delta n
$$

Therefore, 


$$
\Delta n=\frac{\chi^{(2)} E}{n_{0}}
$$

Finally,

$$
\Delta n n_{0}=\chi^{(2)} E
$$

So there is a direct relationship between change in refractive index and the strength of second order nonlinearity. Presence of second order nonlinearity therefore implies presence of a Pockels effect.

Typically in optical frequencies the Kerr coefficient $s$ is few orders of magnitude smaller than Pockels coefficient $r$. Therefore for a Pockels medium, we can write from equation (1.8):

$$
\Delta n=-\frac{1}{2} r n_{0}^{3} E
$$

Using equation (1.19) and (1.20) we readily have:

$$
\chi^{(2)}=-\frac{1}{2} r n_{0}^{4}
$$

\section{5 $\quad \chi^{(2)}$ and $r$ as tensors}

In previous sections we treated nonlinear susceptibility $\chi^{(2)}$ and Pockels coefficient $r$ as scalars, for sake of simplicity. It is important to note the tensorial nature of $\chi^{(2)}, r$ and polarization $P$. Similar to other properties of crystals, $\chi^{(2)}$ has a high degree of dependence on the orientation crystal structure, direction of light propagation and applied electric field. In general terms we can write $\chi^{(2)}$ as a third rank tensor with 27 independent components, leading to the polarization components:

$$
P_{i}^{(2)}(\mathrm{t})=\sum_{j k} \chi_{i j k}^{(2)} E_{j}(t) E_{k}(t)
$$


where $i, j, k=x, y, z$. Some of the tensor components are invariant under permutation of the indices $i$ and $j$, i.e. $\quad \chi_{i j k}^{(2)}=\chi_{j i k}^{(2)}$. Because of this permutation symmetry the 27 components are reduced to only 18 independent components. It is conventional to rename the pair of indices $(i, j), i, j=1,2,3$ as a single index $\mathrm{I}=1,2,3 \ldots, 6$ in accordance with the Table 1 .

\begin{tabular}{|c|c|c|c|c|}
\hline$j:$ & $i$ : & $1(x)$ & $2(y)$ & $3(z)$ \\
\hline $1(x)$ & & 1 & 6 & 5 \\
\hline $2(y)$ & & 6 & 2 & 4 \\
\hline $3(z)$ & & 2 & 4 & 3 \\
\hline
\end{tabular}

Table 1.1 Lookup table for the index I [25].

For example, the pair $(i, j)=(3,2)$ is labeled $I=4$.

The same tensor elements also exist for the Pockels coefficients $r_{i j k}$. Depending on the crystal structure the symmetry of the crystal adds constraints to $r_{i j k}$ where some entries must be zero and some others must be equal, or equal in magnitude. For examples for trigonal $3 m$ point group crystals such as $\mathrm{LiNbO}_{3}$ and $\mathrm{LiTaO}_{3}$ the following Pockels coefficients are allowed:

$$
\left[\begin{array}{ccc}
0 & -r_{22} & r_{13} \\
0 & r_{22} & r_{13} \\
0 & 0 & r_{33} \\
0 & r_{51} & 0 \\
r_{51} & 0 & 0 \\
-r_{22} & 0 & 0
\end{array}\right]
$$

Note that in this case $r_{61}=-r_{22}, r_{42}=r_{51}, r_{12}=-r_{22}$ and $r_{23}=r_{13}$. For LiNbO 3 crystal the Pockels coefficients are $r_{13}=8.6 \mathrm{pm} / \mathrm{V}, \quad r_{22}=3.4 \mathrm{pm} / \mathrm{V}, r_{33}=30.8 \mathrm{pm} / \mathrm{V}$, $r_{51}=28.0 \mathrm{pm} / \mathrm{V}[26]$.

\section{6 $\mathrm{LiNbO}_{3}$ modulator specifications}


In the optical telecommunication industry $\mathrm{LiNbO}_{3}$ modulators are the most widely used types of modulators. Hence, one needs to be familiar with the design and performance characteristics of the typical $\mathrm{LiNbO}_{3}$ modulator, when considering alternatives to this established technology.

The typical optical modulator is formed as a Mach-Zehnder Interferometer (MZI) on a $\mathrm{LiNbO}_{3}$ substrate. Schematic of a MZI is illustrated in Figure 1.5. The input laser beam enters a beam splitter which divides the input power to two equal portions. The divided power propagates in two "arms" of the MZI. At least one of the arms is an active arm that can change the phase of the propagating light. The beams from both arms combine in the combiner. If the phases of the beams from arm 1 and arm 2 are exactly equal (or a multiple of $\pi$ ) then the light interferes constructively in the combiner and propagates as a single mode in the output waveguide from the MZI. If the light traveling through the two arms is out of phase at the combiner, interference in the forward direction is destructive and no light is guided in the output waveguide.

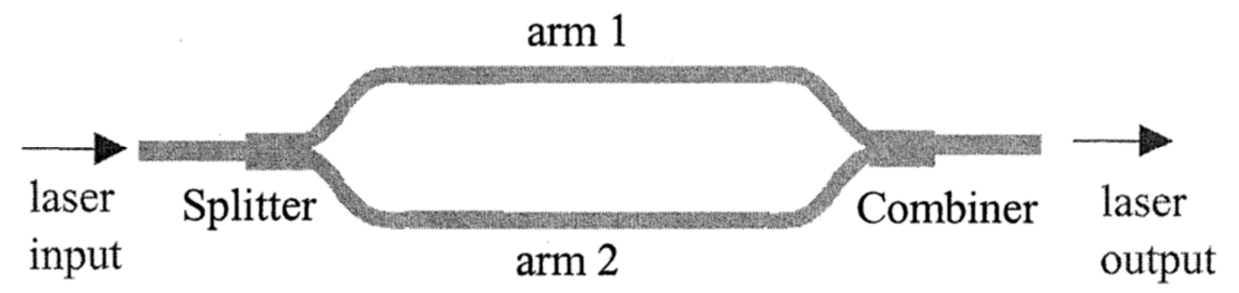

Figure 1.5 Schematic of a Mach-Zehnder Interferometer [26].

The waveguides are formed by diffusing titanium (Ti) on a Lithium Niobate substrate using metal evaporation, photolithography and liftoff techniques [26]. Titanium changes local refractive index of the substrate by $\sim 0.01$, which is ideal for single mode optical waveguides. Typically waveguide stripes are about $3-8 \mu \mathrm{m}$ wide and $\sim 0.1 \mu \mathrm{m}$ thick and 
have very low loss of $<0.2 \mathrm{~dB} / \mathrm{cm}$. The mode profile is also a good match with the single mode fiber.

Performance metrics of a typical $\mathrm{LiNbO}_{3}$ electro-optic modulator include [26], [27]:

- Modulation speed: due to atomic nature of the Pockels effect the response times of the material are ultrafast; in principle speed is only limited by electrode design. Typical frequencies are 10 to $100 \mathrm{GHz}$.

- Modulation depth or extinction ratio is expressed in $\mathrm{dB}$ using $10 \log \left(\mathrm{I}_{\min } / \mathrm{I}_{\max }\right)$. Typical value is $20 \mathrm{~dB}$.

- Fiber-to-fiber insertion loss including reflection, absorption, and mode coupling losses: Typical value is $4-5 \mathrm{~dB}$.

- Power consumption: the energy expanded in producing each bit of data target: 1 pJ/bit.

- $V_{\pi} L$ : Voltage required for $\pi$ radian phase shift. Typically around: 6-12 V-cm. $\mathrm{LiNbO}_{3}$ physical limit $\approx 3.6 \mathrm{~V}-\mathrm{cm}$.

- Optical bandwidth: operational wavelength range. $\mathrm{LiNbO}_{3}$ is transparent from 0.33 to $4.5 \mu \mathrm{m}$. Typical working band for commercial telecom modulators: 1525 $\mathrm{nm}$ to $1605 \mathrm{~nm}$.

- Footprint. Packages modulators have dimensions of: $2-5 \times 1-2 \mathrm{~cm}^{2}$ 


\section{Chapter: Literature Review}

In this chapter we will discuss the important past work done on strain induced second order optical nonlinearity in silicon.

\subsection{Second harmonic generation at silicon surfaces}

As discussed in Chapter 1, in materials with inversion symmetry, such as $\mathrm{Si}$ and Ge, second order nonlinearity $\chi^{(2)}$ is not allowed. In this case the process of SHG is governed by higher-order nonlinearities that are very weak [22]. However in real structures a stronger $\chi^{(2)}$ is induced by two factors, one is the removal of the symmetry at the surface and the other is the physical deformation of the material (inhomogeneous strain) near the surface.

Unlike the Pockels effect which was discovered only recently in silicon, SHG from silicon surface has been studied extensively for decades [19], [22]. In 1989 Govorkov et al. reported the observation of two orders of magnitude enhancement in induced $\chi^{(2)}$ in silicon wafers that were inhomogeneously strained [22] compared to unstrained wafers. Strain was applied by ion-implantation of $\mathrm{Si}$, thermal-oxidization of $\mathrm{Si}$, and deposition of silicide on Si samples. They also reported strong dependence of reflected SH signals on sample orientation and azimuthal angle.

More recently, in 2010, Schriever et al. [15] presented their theoretical and experimental study of thermal oxide strain effects of silicon surface on the second order susceptibility $\chi^{(2)}$. They concluded that the value of the induced $\chi^{(2)}$ is directly dependent on the amount of mechanical strain. The theoretical model is based on the Elasticity Theory which expresses the stress $\sigma$ at a flat surface of a material as:

$$
\sigma(\mathrm{y})=\sigma_{0} \exp (-y / d)
$$


where $\sigma_{0}$ is initial stress at the interface, $d$ is the characteristic stress decay on the order of $10 \mathrm{~nm}$, and $y$ is distance from the interface. Adjusting the molecular model developed by [22] and [28] to include strain effects, they concluded that second order nonlinearity resulting from deformation of the silicon surface $\chi_{\text {def }}^{(2)}$ is directly proportional to the $\sigma_{0}$ and $d$ :

$$
\chi_{\text {def }}^{(2)} \propto \frac{\sigma_{0}}{d}
$$

In order to experimentally measure $\chi_{\text {def }}^{(2)}$ and verify equation (2.2) Schriever et al. measured SH signals reflected from surface of silicon wafers with various strain levels. Figure 2.1 (a) shows the schematic of the experimental setup used. Assume a laser beam with frequency $\omega$ incidents on the surface of a silicon crystal. The reflected second harmonic beam with frequency $2 \omega$ has dependence on azimuthal angle $\Phi$. Detecting the SH signal produces a pattern shown in Figure 2.1 (b).

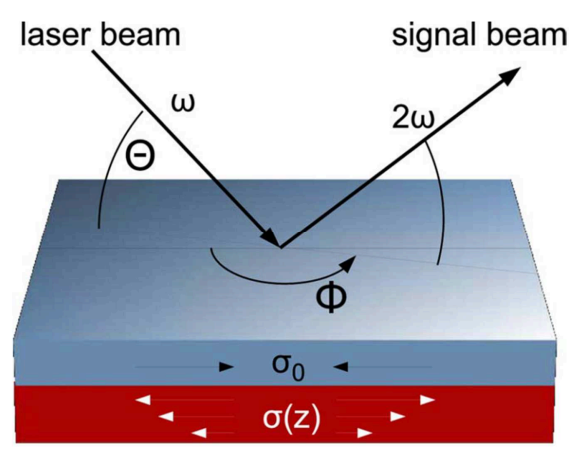

(a)

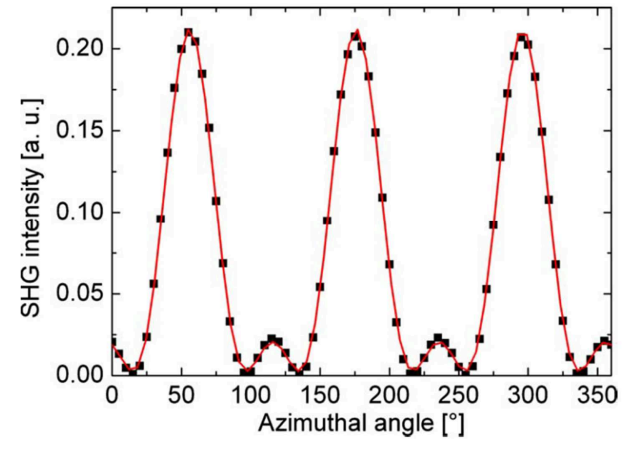

(b)

Figure 2.1 Second harmonic beams reflected from silicon surface.

(a) Experimental setup for measurement of SHG's reflected signal on the azimuthal angle $\Phi$. (b)

Detected SH signal as a function of azimthal angle [15] 
The phenomenological theory developed by Sipe et al. in [29] gives an expression for dependence of SH signal intensity on symmetry and azimuthal angle $\phi$. For a (111)oriented silicon crystal:

$$
I_{p p}^{S H G} \propto\left|a_{p p}+c_{p p} \cos (3 \phi)\right|^{2} I_{p, 0}^{2}
$$

where $a_{p p}$ and $c_{p p}$ contain the Fresnel coefficients and the quadrupolar contributions, $\phi$ is the incident angle and $\mathrm{I}_{\mathrm{p}, 0}$ is p-polarized fundamental beam. In addition, $a_{p p}$ has dependence on $\chi^{(2)}$. They were able to observe the linear dependence of coefficient $a_{p p}$ (and thus $\chi^{(2)}$ ) on stress by comparing samples with various amounts of stress as shown in Figure 2.2.

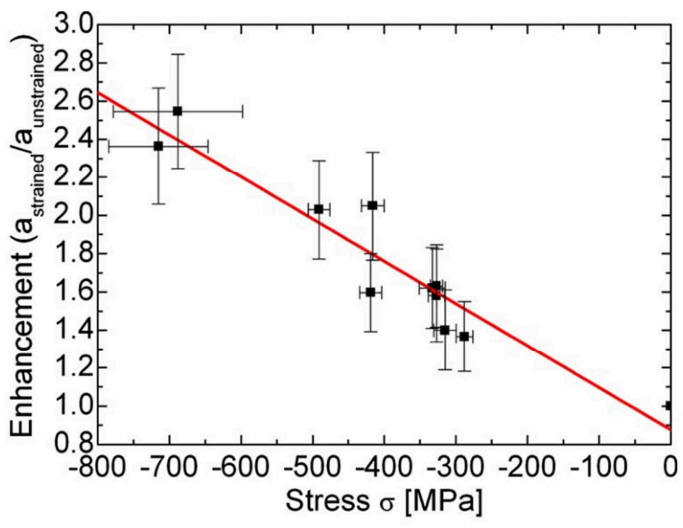

Figure 2.2 Linear dependence of second order non-linearity coefficients $a_{p p}$ and $c_{p p}$ on stress [15].

\subsection{Second harmonic generation in silicon waveguides}

In 2012, Cazzanelli et al. demonstrated that generation of second harmonic signals by strain-induced second-order nonlinearity in silicon waveguides is possible. The $2 \mu \mathrm{m}$ SOI strained waveguides produced second harmonic signals of the incident beam [14]. A layer of $\mathrm{Si}_{3} \mathrm{~N}_{4}$ was deposited directly on the waveguide as the straining layer, as seen in Figure 2.3 (a). Three sets of waveguides were fabricated, each strained by a different 
amount. The highest $\chi^{(2)}=40 \pm 30 \mathrm{pm} / \mathrm{V}$ at $2300 \mathrm{~nm}$ was observed for a $10.7 \mu \mathrm{m}$ wide waveguide with $+1.2 \mathrm{GPa}$ stress in the $\mathrm{Si}_{3} \mathrm{~N}_{4}$ straining layer. The difference in the stress applied from the top silicon nitride and the bottom BOX layer, typically -300 MPa, causes a strong gradient to be present inside the silicon core. To demonstrate the induced $\chi^{(2)}$, second harmonic generation (SHG) experiments were carried out. Figure 2.3 (b) shows typical transmission signal recorded. The strong peak is at $\lambda=2100 \mathrm{~nm}$ which is the pump frequency. The SH signature at $\lambda / 2=1050 \mathrm{~nm}$ can be seen. The shift in the $\mathrm{SH}$ peak with pump frequency is demonstrated in Figure 2.3 (c).

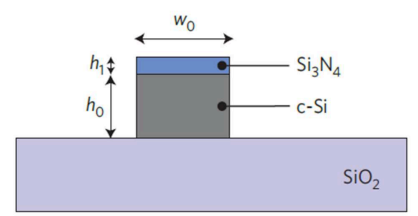

(a)

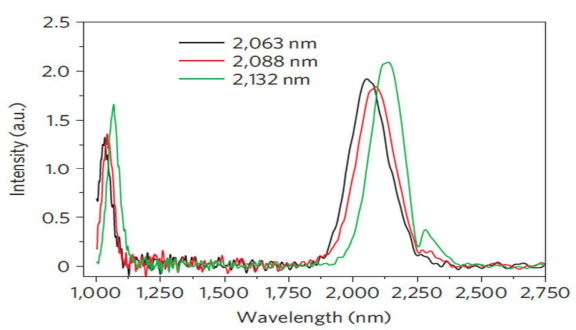

(b)

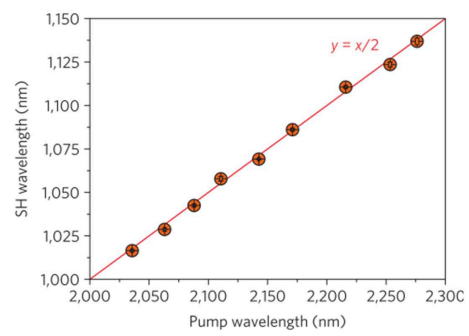

(c)

Figure 2.3 Strained silicon waveguides used to produce second harmonic signals.

(a) Silicon waveguide has height of $2.0 \mu \mathrm{m}$. The width of the silicon waveguide is $2 \mu \mathrm{m}$ to $11.7 \mu \mathrm{m}$ and the height of the $\mathrm{Si}_{3} \mathrm{~N}_{4}$ varies between 150 to $500 \mathrm{~nm}$. (b) Experimental result showing SHG for various wavelengths; SH signature can be seen around $1050 \mathrm{~nm}$. (c) SH wavelength as a function of pump wavelength, showing strict linear relation between the two [7].

In addition to experimental demonstration of SHG in silicon waveguides, Cazzanelli et al also carried out ab-initio calculations of strain induced $\chi^{(2)}$ in silicon crystals. Timedependent density-functional theory in a super-cell approximation with periodic 
boundary conditions was used. A unit cell of $16 \mathrm{Si}$ atoms (4 sets of 4 atoms) was considered initially at their bulk centro-symmetric positions; see Figure 2.4 (a). The unit cell was then repeated in whole space along the three lattice vectors $a_{1}, a_{2}, a_{3}$, as shown in Figure 2.4 (b). For this arrangement of atoms the obtained $\chi^{(2)}$ vanishes, as expected, due to central symmetry.

To simulate strained silicon lattice, 2 sets of 4 atoms were moved from their unstrained position, representing tensile and compressive strains. In Figure 2.4 (c) the red balls/sticks and orange balls/sticks represent the tensile and compressively strained sets of atoms, respectively. The lattice vectors are modified to correspond to the new strained cell. The unit cell was then repeated in the whole space, as seen in Figure 2.4 (d). Two classes of strained cells where examined: $\mathrm{US}_{\mathrm{c}} \mathrm{US}_{\mathrm{t}}$ (unstrained-compressed-unstrainedtensile) and $\mathrm{US}_{\mathrm{t}} \mathrm{US}_{\mathrm{t}}$ (unstrained-tensile-unstrained-tensile). It was observed non-vanishing $\chi^{(2)}$ exist whenever the cell is strained. The best situation was obtained when tensile and compressive strains existed in the unit cell that lead to higher strain gradient. In addition moving $S_{t} / S_{c}$ sets of atoms along (001) direction only, results in $\chi^{(2)}$ of less than $0.5 \mathrm{pm} / \mathrm{V}$ at $2500 \mathrm{~nm}$. However moving $\mathrm{S}_{\mathrm{t}} / \mathrm{S}_{\mathrm{c}}$ sets of atoms also in (110) direction (which increases strain inhomogeneity) increases $\chi^{(2)}$ to about $200 \mathrm{pm} / \mathrm{V}$ at $1800 \mathrm{~nm}$, signifying the effect of biaxial stress in strain induced $\chi^{(2)}$. 


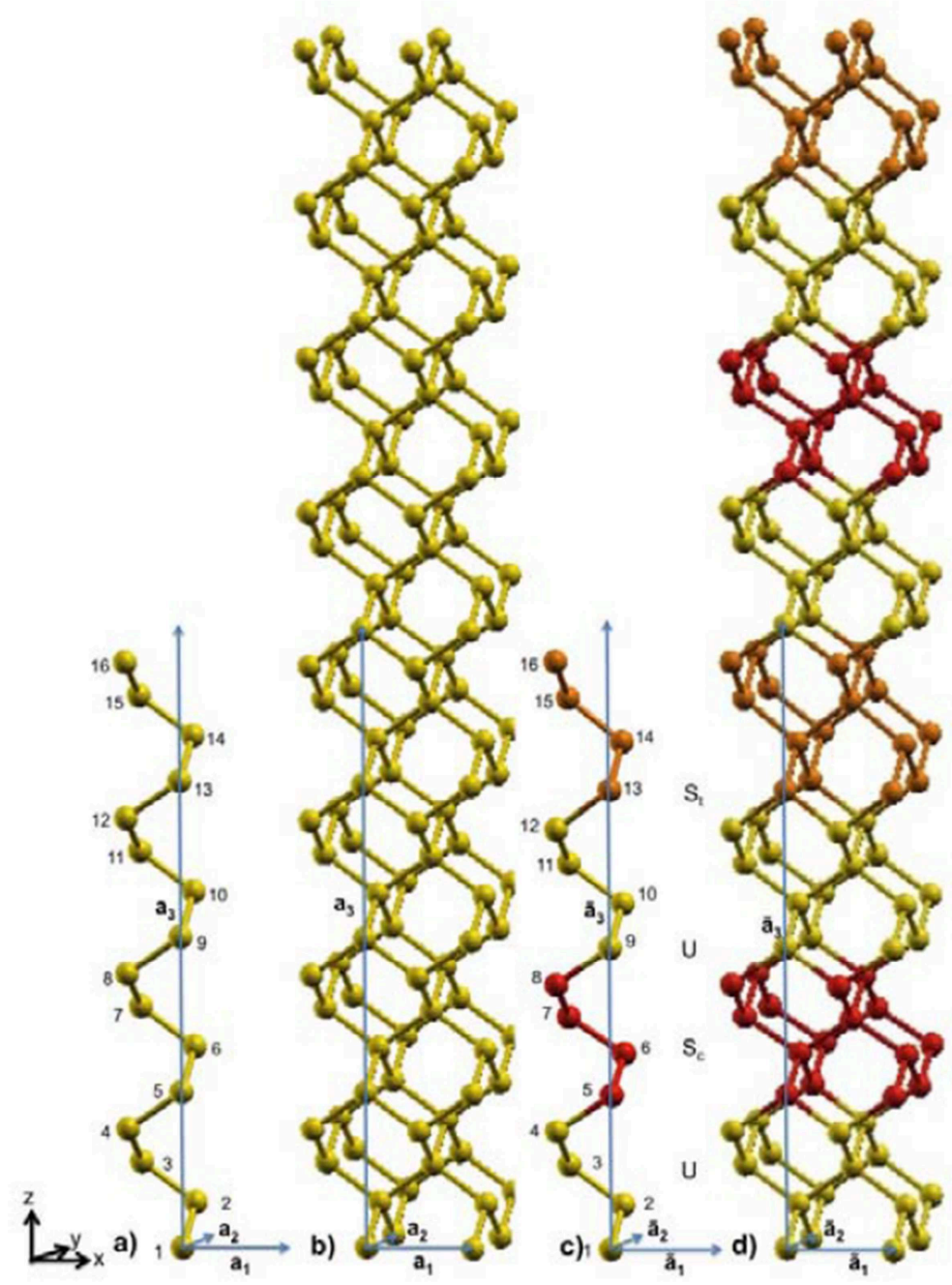

Figure 2.4 Schematic of cells used for ab-initio simulations to calculate strain induced $\chi^{(2)}$ in silicon crystal.

(a) Unit cell of $16 \mathrm{Si}$ atoms for unstrained bulk and lattice vectors $\mathrm{a}_{1}, \mathrm{a}_{2}$ and $\mathrm{a}_{3}$. (b) Repeated 2 cells for each direction along the lattice vectors. (c) Unit cell of 16 atoms for strained bulk and the modified lattice vectors $\tilde{a}_{1}, \tilde{a}_{2}$ and $\tilde{a}_{3}$. (d) Repeated 2 cells for each direction along the lattice vectors. Yellow balls/sticks unstrained silicon regions, orange balls/sticks are tensile strained regions and red balls/sticks are compressively strained regions [14]. 


\subsection{Strain induced Pockels effect in silicon modulators}

The first reported observation of electro-optic effect in a silicon waveguide was made by Jacobsen et al. [7]. They used a $\mathrm{Si}_{3} \mathrm{~N}_{4}$ layer to induce mechanical stress on to a $220 \mathrm{~nm}$ thick SOI waveguide. The asymmetrical mechanical stress breaks the centro-symmetry in

the bulk silicon waveguide and induces second order nonlinearity $\chi_{S i}^{(2)}$. The straining layer is compressively stressed, which leads to tensile strain at the surface of the silicon waveguide and deforms the top of the waveguide, as schematically shown in Figure 2.5.

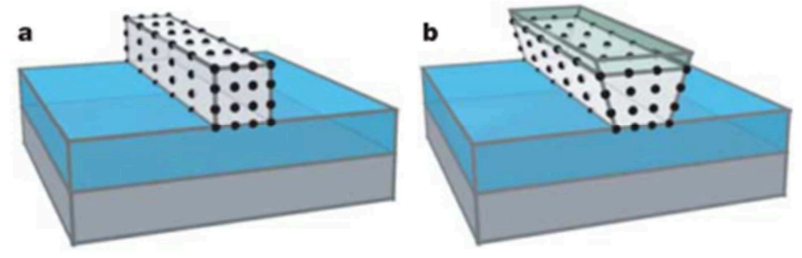

Figure 2.5 Schematic of effect of straining layer on a silicon waveguide.

(a) Unstrained silicon waveguide; (b) Strained silicon waveguide. The deformation breaks the centrosymmetry and induces second order nonlinearity $\chi^{(2)}[7]$.

By applying an electric field across the waveguide the refractive index of the waveguide changes which leads to phase change in the propagating light. To observe the phase shift two waveguides were put in two arms of a Mach-Zehnder Interferometer (MZI). At the input of the MZI light couples to both arms. Electric field is applied to one arm which causes a phase shift in propagating light. MZI Light beams from both arms are coupled back into an output waveguide where they interfere constructively or destructively depending on phase difference of the two beams. This is schematically shown in Figure 2.6 (a). 
In order to enhance the observed electro-optic effect a Photonic Crystal Wire (PCW) was used in the active arm. The PCW permanently enhances the group index, $\mathrm{n}_{\mathrm{g}}$ [30]. The enhanced second order susceptibility then equals:

$$
\chi_{\text {enh }}^{(2)}=\frac{n_{g}}{n} \chi^{(2)}
$$

In order to ensure that the observed $\chi^{(2)}$ originates from the Pockels effect in the silicon waveguide and not the charge movement inside $\mathrm{Si}_{3} \mathrm{~N}_{4}$ layer, a $1 \mu \mathrm{m}$ thick silicon dioxide spacing layer was deposited on top of the silicon waveguide, separating it from the $\mathrm{Si}_{3} \mathrm{~N}_{4}$ straining layer. The spacing layer has stress of $+300 \mathrm{MPa}$. The silicon nitride layer has stress of $-1 \mathrm{GPa}$ and is $750 \mathrm{~nm}$ thick. The induced $\chi^{(2)} \approx 15 \mathrm{pm} / \mathrm{V}$ was observed.

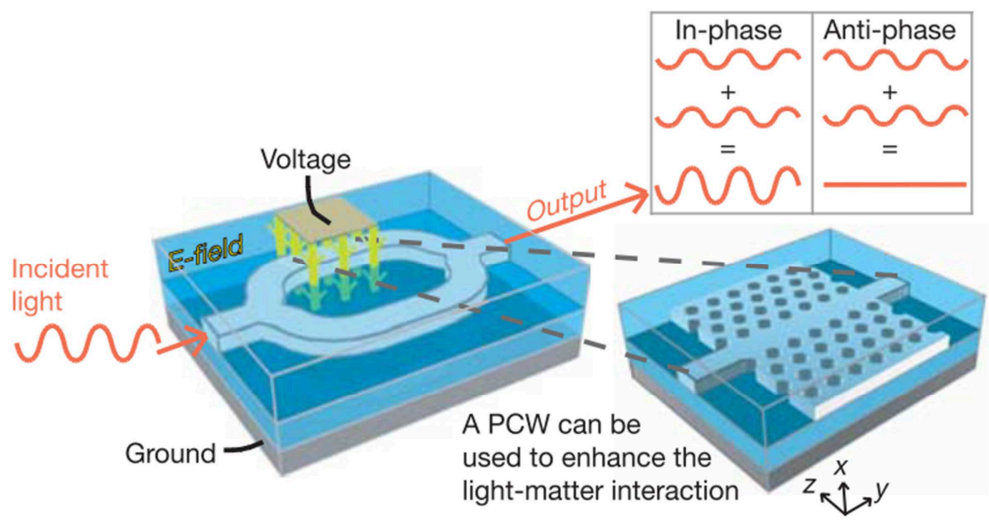

Figure 2.6 Schematic of (a) the Mach-Zehnder Interferometer used to demonstrate the Pockels effect and (b) Photonic Crystal Wire (PCW) used to ehance $\chi^{(2)}[7]$.

The stress originating from the buried oxide (BOX) $\mathrm{SiO}_{2}$ layer also induces $\chi_{\text {enh }}^{(2)}$. The blue dots in Figure 2.7 indicate the observed $\chi_{e n h}^{(2)}$ for waveguides without the straining layer. The obtained $\chi_{e n h}^{(2)}$ is due to strain from the BOX layer. The black line shows $\chi_{e n h}^{(2)}$ when $\mathrm{Si}_{3} \mathrm{~N}_{4}$ straining layer is deposited. The difference between the magnitude of the blue dots and solid line shows the improvement of $\chi_{e n h}^{(2)}$ due to straining layer. 


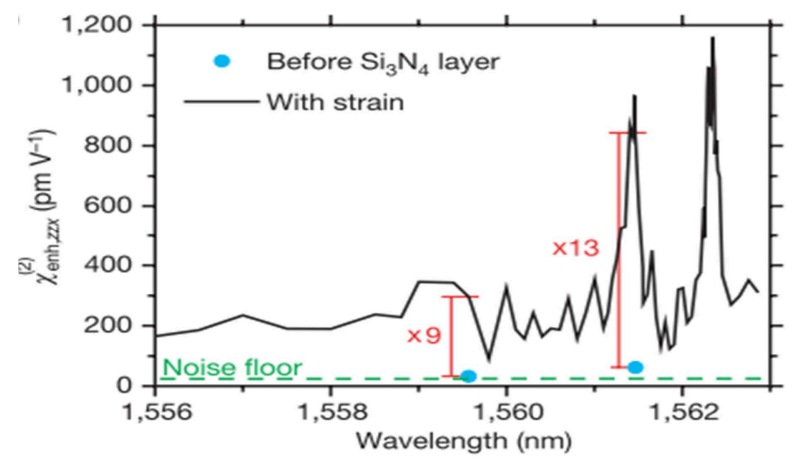

Figure 2.7 $\chi_{e n h}^{(2)}$ in silicon waveguide as function of wavelength before and after deposition of straining layer.

$\chi_{\text {enh }}^{(2)}$ detected before application of $\mathrm{Si}_{3} \mathrm{~N}_{4}$ straining layer is due to stress from the BOX underneath the waveguide. An order of magnitude imrpovement $\chi_{e n h}^{(2)}$ of is observed when straining layer is applied

Additional experiments were also carried out to ensure the observed effect is caused by strain and not by other effects previously discovered in silicon, such as thermo-optic, photo-elastic, photo-electric and interface effects. All possibilities were ruled out by appropriate experiments.

The first fully integrated electro-optic modulator based on strained silicon was demonstrated by Chmielak et al. in 2011 [9]. This design is based on 300 to $500 \mathrm{~nm}$ wide rib waveguides on $220 \mathrm{~nm} \mathrm{SOI}$. The $\mathrm{Si}_{3} \mathrm{~N}_{4}$ straining layer is directly deposited on the core and it covers the entire top, sidewalls and the ribs of the silicon waveguide, see Figure 2.8. The etch depth is $175 \mathrm{~nm}$ and thickness of the straining layer is $350 \mathrm{~nm}$. An important advantage of rib waveguide over channel structure is the etch depth parameters which becomes an extra degree of freedom in designing the waveguide. The residual stress in the silicon nitride layer is $+1 \mathrm{GPa}$ as reported in a later paper [13]. $\mathrm{SiO}_{2}$ cladding 
layer is deposited on top of the $\mathrm{Si}_{3} \mathrm{~N}_{4}$ layer to provide electrical isolation from the waveguide and to allow a platform to deposit $\mathrm{Ti} / \mathrm{Au}$-electrodes.

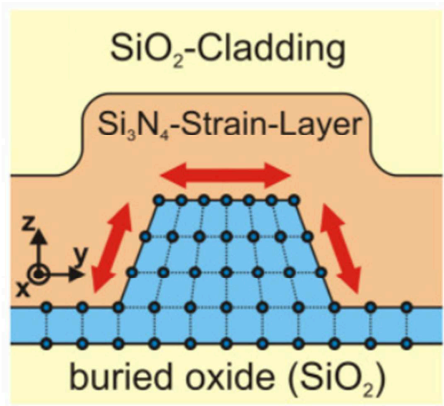

(a)

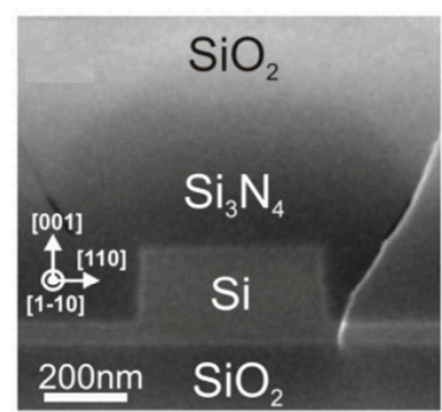

(b)

Figure 2.8 Cross section of a rib waveguide with $\mathrm{Si}_{3} \mathrm{~N}_{4}$ straining layer.

(a) Schematic cross-section of waveguides. (b) Scanning Electron Microscope (SEM) image of a typical waveguide in [9].

In this work modulators were designed as Mach-Zehnder interferometer (MZI) devices. The length difference between the two arms is $\Delta \mathrm{L}=50 \mu \mathrm{m}$. The MZI helps detection of effective index change $n_{\text {eff }}$ due to applied DC electric field. Figure 2.9 (a) shows the schematic cross-section of the induced electric field by the electrodes. The $x, y$ and $z$ axis coincide with the [110], [001], and [1-10] crystalline planes, respectively. Since the dc electric field is along the $y$ direction and electric field of the TE mode is along $x$ direction, the only non-vanishing $\mathrm{E}_{\mathrm{Opt}, i} \cdot \mathrm{E}_{\mathrm{Mod}, j}$ term is when $i=x$ and $j=y$. Therefore the relevant component of the second order nonlinearity in equation (1.22) is $\chi_{\mathrm{xxy}}^{(2)}$.

Figure 2.9 (b) shows how the push-pull electrode configuration, which applies opposite polarity on opposite arms, is implemented. This helps to reduce the device foot print. Figure 2.9 (c) shows a typical spectrum obtained from an output arm of the MZI using 0, +30 and $-30 \mathrm{~V}$ modulation voltage.

Figure 2.9 (d) correlates the applied voltage to shift in peak wavelength around $1575 \mathrm{~nm}$. The corresponding $\mathrm{E}_{\mathrm{Mod}}$ and $\Delta \mathrm{n}_{\mathrm{eff}}$ are included on the upper and the right axis, 
respectively. By calculating the confinement factor of the waveguide the index change

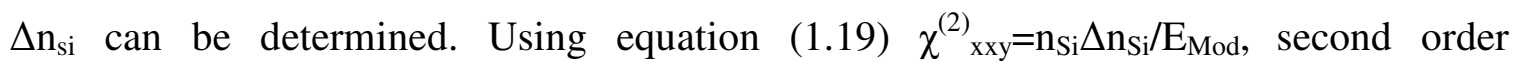
nonlinearity is calculated. The electro-optical characterization reveals highest second order nonlinearity in silicon reported to date $\chi_{S i}^{(2)}=190 \mathrm{pm} / \mathrm{V}$, which is for the $300 \mathrm{~nm}$ waveguide. It is worth noticing due to small size of the waveguide the optical confinement in silicon core is only $\Gamma=42 \%$. The portion of the mode outside the silicon core is not be affected by $\chi_{S i}^{(2)}$ therefore the effective waveguide $\chi^{(2)} \approx 79.8 \mathrm{pm} / \mathrm{V}$.

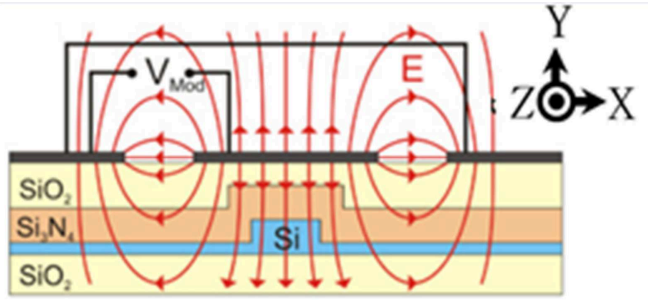

(a)

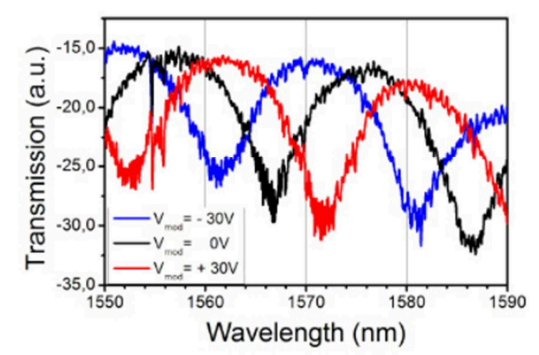

(c)

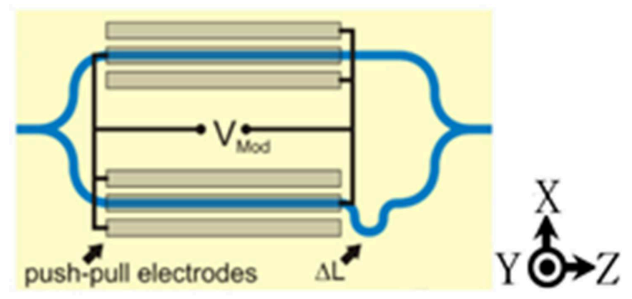

(b)

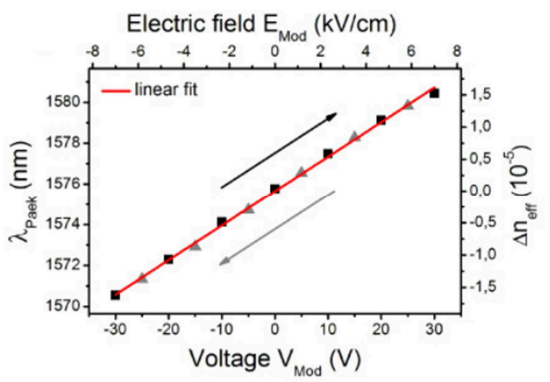

(d)

Figure 2.9 First fully integrated strain induced Pockels effect modulator.

(a) Schematic cross-section of waveguides in the arms of the MZI. (b) Top view schematic of the MZI structure. (c) Observed spectral shifts spectrum for $+30 \mathrm{~V}$ and $-30 \mathrm{~V}$ modulation voltages. (d) Peak wavelength shift as a function of applied modulation voltage, corresponding change in effective index and applied electric field [9]. 
Spatially resolved nonlinear optical mappings were conducted using micro-Raman spectroscopy. The results indicate high degrees of strain inhomogeneity are present at the bottom corners of the core.

\subsection{D centro-symmetric lattice model}

Although theoretical studies of strain induced Pockels effect in silicon are not found to predict $\chi_{\mathrm{Si}}^{(2)}$ in silicon waveguides correctly, there are general conclusions that can be made with the help of the models. In [10], Hon et al. used a simple 2D centro-symmetric lattice model to examine strain induced $E$ field inside silicon crystal. The model assumes $E$ is dominated only by 4 nearest silicon atoms that are equally distanced, by silicon bond length $(\sim 0.433 \times$ lattice constant $)$ as shown in Figure 2.10 . The centro-symmetry is broken by application of strain in $x$ direction with a gradient in $y$, i.e. $\partial \varepsilon_{\mathrm{xx}} / \partial \mathrm{y}$, similar to what occurs in asymmetrically strained silicon waveguides. The displacement of atoms from their centro-symmetric configuration, dotted circles, induces a net electric field $E_{n e t}$ in the $y$ direction.

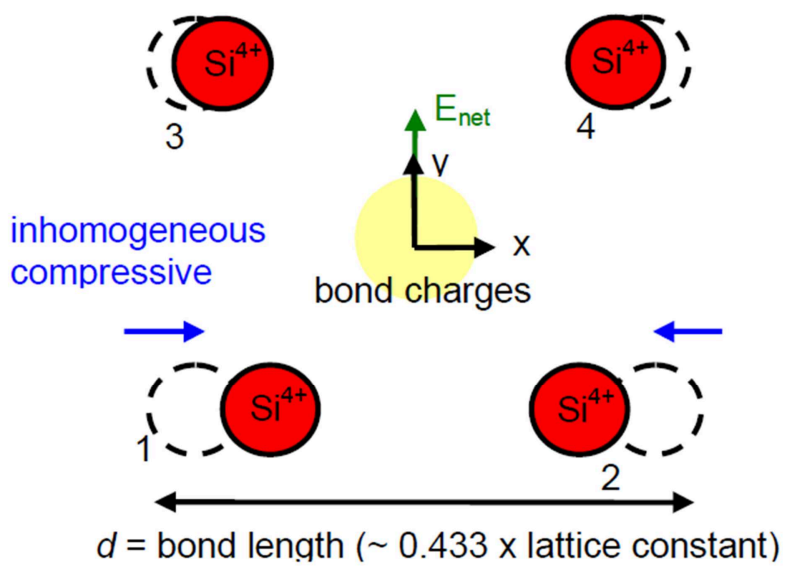

Figure 2.10 Schematic of a $2 \mathrm{D}$ centro-symmetric silicon lattice structure under inhomogeneous stress [10]. 
For simplicity they assumed the top atoms are fixed and the bottom atoms are strained, then they used Coulomb's law to express the $E_{n e t}(y)$ at the center of the lattice as:

$$
E_{n e t}(y)=\frac{2 e}{\pi \varepsilon_{0}}\left(\frac{(d / 2+y)}{\sqrt[3]{\left(\frac{d}{2}-\Delta S d \frac{d}{2}\right)^{2}+\left(\frac{d}{2}+y\right)^{2}}}+\frac{(d / 2-y)}{\sqrt[3]{\left(\frac{d}{2}\right)^{2}+\left(\frac{d}{2}-y\right)^{2}}}\right)
$$

where the $\Delta \mathrm{S}=\partial \varepsilon_{\mathrm{xx}} / \partial \mathrm{y}$ is the strain gradient. The first term in the brackets is the $E_{n e t}$ due to the bottom atoms and the second term is $E_{n e t}$ due to the top atoms. Since $E_{\text {net }}$ is parallel with the electric modulation field in the modulator configuration considered in Figure 2.9 (c), we conclude $\partial \varepsilon_{x x} / \partial y$ is the main origin of $\chi_{x x y}^{(2)}$ [10], [13]. The derivation of equation (2.5) is available in Appendix C . 


\section{Chapter: Strain Simulations}

In this chapter we will discuss how the stress and strain distribution for silicon waveguides and nanowires are calculated.

\subsection{Stress and Strain in SOI waveguides}

\subsubsection{Origins of stress in SOI waveguides}

Figure 3.1 shows the configuration of a typical strained SOI wafer. It consists of a single straining layer (e.g. $\mathrm{Si}_{3} \mathrm{~N}_{4}, \mathrm{a}-\mathrm{Si}, \mathrm{SiGe}, \mathrm{SiC}$, etc.) with thickness of typically between 150 $\mathrm{nm}$ and $500 \mathrm{~nm}$, a crystalline silicon core layer between $220 \mathrm{~nm}$ and $2.2 \mu \mathrm{m}$ thick, a buried oxide (BOX) layer about $3 \mu \mathrm{m}$ thick that acts as the bottom cladding, and the silicon substrate with several hundred microns thickness. The straining layer at the top can act as the top cladding layer if the refractive index is lower than that of silicon.

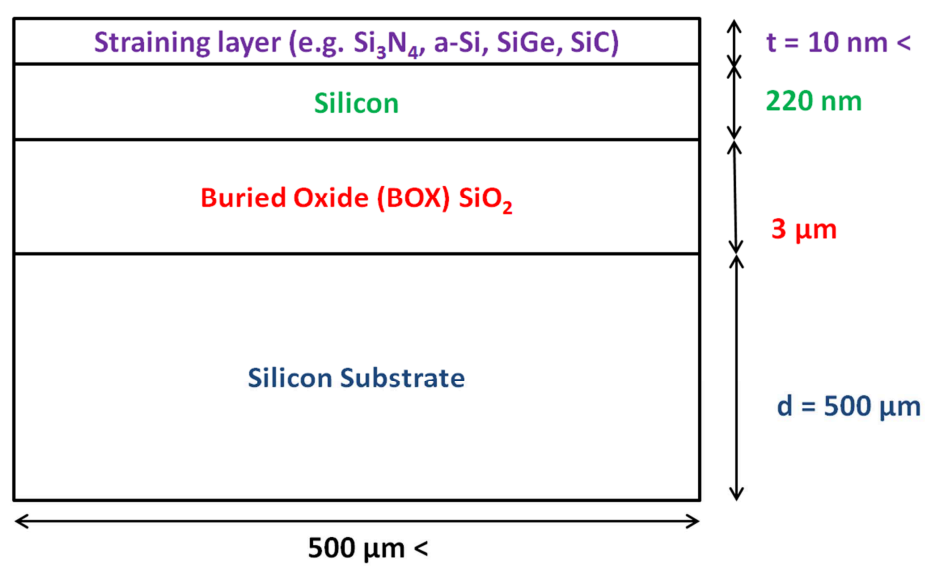

Figure 3.1 Typical cross section of SOI platform with a straining layer.

Optical or electron beam (e-beam) lithography is used to define an etch mask. In order to form rib or channel waveguides the silicon core layer is etched and the remaining silicon forms 2 dimensional waveguides. Then, chemical vapor deposition (CVD) or epitaxial techniques are used to deposit a cladding layer and/or stressor layer. Figure 3.2 shows the cross section of a rib waveguide. Geometrical parameters for a rib waveguide include: 
core width $(\mathrm{W})$, core height $(\mathrm{H})$, etch depth (ED) and upper cladding thickness (CT). In many applications the waveguides are invariant along the propagation direction, $z$, hence a two dimensional treatment of the problem suffices.

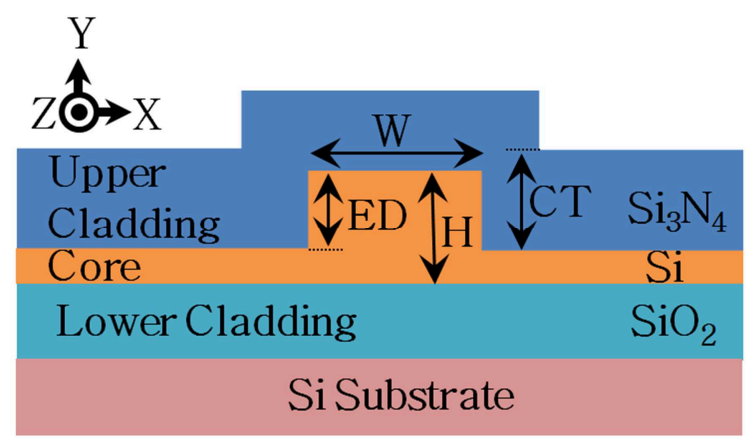

Figure 3.2 The cross section and geometrical parameters of a rib waveguide with silicon nitride upper cladding layer on SOI platform.

The amount of stress and strain in SOI waveguides is governed by the thermal stress as well as the intrinsic (or residual) strain:

$$
\sigma_{\text {film }}=\sigma_{t h}+\sigma_{i n}
$$

where $\sigma_{\text {fim }}$ is average film stress in the $\mathrm{Si}_{3} \mathrm{~N}_{4}$ straining layer, $\sigma_{t h}$ is thermal stress and $\sigma_{\text {in }}$ is intrinsic stress. Thermal stress results from the fact that materials of different layers have different thermal expansion coefficients and there is generally a difference between the deposition and operating temperatures. During the cool down process layers shrink at different rates and therefore they exert force upon each other.

Intrinsic stress can exist in epitaxial growth if there is a lattice mismatch between silicon core layer and the deposited layer. Intrinsic stress can also originate from the residual stress resulting from deposition process. Materials such as silicon nitride and amorphous silicon that are deposited on the waveguides using Plasma Enhanced Chemical Vapor Deposition (PECVD) and Low Pressure Chemical Vapor Deposition (LPCVD) can have 
substantial intrinsic stress depending on deposition conditions such as pressure and temperature. For examples Figure 3.3 shows variation of both intrinsic and total stress as functions of low frequency power density in the LPCVD process [31]. The difference between total and intrinsic stress is due to thermal stress.

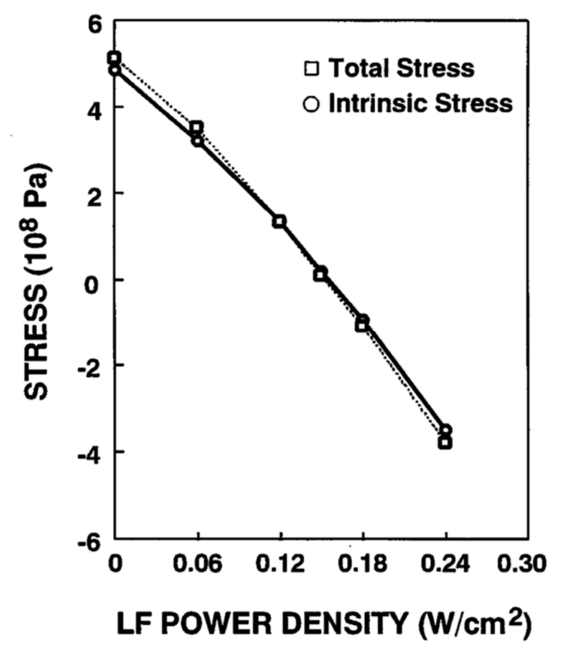

Figure 3.3 Intrinsic and total stress of a $\mathrm{Si}_{3} \mathrm{~N}_{4}$ thin film as functions of low frequency power density [31].

\subsubsection{Stress/Strain relationship}

The stress and strain components are related as follows:

$$
\left[\begin{array}{l}
\varepsilon_{x} \\
\varepsilon_{y} \\
\varepsilon_{z}
\end{array}\right]=\frac{1}{E}\left(\left[\begin{array}{ccc}
1 & -v & -v \\
-v & 1 & -v \\
-v & -v & 1
\end{array}\right] \quad\left[\begin{array}{c}
\sigma_{x} \\
\sigma_{y} \\
\sigma_{z}
\end{array}\right]\right)+\alpha \Delta T
$$

where $\varepsilon_{\mathrm{i}}$ and $\sigma_{\mathrm{i}}$ are stress and strain components along the $x, y, z$ directions, respectively. $E$ is the Young's modulus, $\alpha$ is thermal expansion coefficient, $\Delta T$ is difference between the operating temperature and reference temperature $\left(\Delta T=T_{0}-T_{r e f}\right) . v$ is the Poisson ratio. Equation (3.2) shows that the total strain consists of the elastic (first term) and the thermal-induced (second term) strain. 
By inverting (3.2) the expression for the stress distribution can be obtained as [32]:

$$
\left[\begin{array}{c}
\sigma_{x} \\
\sigma_{y} \\
\sigma_{z}
\end{array}\right]=\frac{E}{(1+v)(1-2 v)}\left(\left[\begin{array}{ccc}
1-v & v & v \\
v & 1-v & v \\
v & v & 1-v
\end{array}\right]\left[\begin{array}{c}
\varepsilon_{x} \\
\varepsilon_{y} \\
\varepsilon_{z}
\end{array}\right]\right)+\frac{E \alpha \Delta T}{1-2 v}
$$

Material properties for various layers of the structures used in this work are summarized in Table 3.1. Thermal expansion coefficient (TEC) varies by temperature. Since the change is nearly linear over a temperature range, typically an average value is assumed. Physical properties of $\mathrm{Si}, \mathrm{SiO}_{2}$ (in BOX layer), and epitaxially grown $\mathrm{SiGe}$ are very well studied and reported values are consistent throughout literature [33], [34]. However, properties of amorphous silicon (a-Si) and $\mathrm{Si}_{3} \mathrm{~N}_{4}$ vary widely depending on the deposition conditions, and a large range of values have been reported [31], [33]-[40].

\begin{tabular}{ccccc}
\hline Material & $\begin{array}{c}\text { Young's } \\
\text { modulus } E \\
(\mathrm{GPa})\end{array}$ & $\begin{array}{c}\text { Poisson's } \\
\text { ratio, } v\end{array}$ & $\begin{array}{c}\text { Thermal expansion } \\
\text { coefficient } \alpha \\
\left(10^{-6} \mathrm{~K}^{-1}\right)\end{array}$ & $\begin{array}{c}\text { Refractive } \\
\text { index }^{a} \\
n\end{array}$ \\
\hline \hline $\mathrm{Si}$ & 130 & 0.27 & 3.6 & 3.476 \\
$\mathrm{SiO}_{2}$ & 76.7 & 0.186 & 0.54 & 1.444 \\
$\mathrm{Si}_{3} \mathrm{~N}_{4}$ & $63-1033$ & $0.25-0.27$ & $2.3-4.0$ & $1.98-2.1$ \\
$\mathrm{Si}_{(0.8)} \mathrm{Ge}_{(0.2)}$ & 124.6 & 0.266 & 4.04 & 3.636 \\
$\alpha-\mathrm{Si}$ & $14-134$ & $0.24-0.27$ & $1.0-4.0$ & 3.55 \\
\hline
\end{tabular}

${ }^{a}$ Measured at wavelength $\lambda=1550 \mathrm{~nm}$

Table 3.1: Physical properties for material typically used for various layers of strained silicon waveguides [31], [33]-[40], [53].

Given the material properties and the geometry of the structures, the temporal evolution of the stress within the waveguide structure is calculated. TEC of silicon is about 7 times larger than that of silicon dioxide, this results in a compressive stress in the oxide layer 
when the structures is cooled down to room temperature. Typically stress of the BOX layer is around -300 MPa [32].

In experiments film stress is calculated by measuring wafer bending after deposition of straining layer and by using the Stoney equation:

$$
\sigma_{\text {film }}=\frac{E h^{2}}{(1-v) 6 R t}
$$

where $\frac{E}{(1-v)}$ is the biaxial elastic modulus of the substrate, $h$ is the height of substrate thickness, $R$ is bend radius, $t$ is the film thickness and $\sigma_{\text {film }}$ is average film stress [32].

In order to carry out simulations and compare calculations with experimental results sometimes temperature difference between $T_{r e f}$ and $T_{0}(\Delta T)$ needs to be set to an artificial value in the simulation software to achieve the experimentally measured stress levels. It has been previously shown using $T_{\text {ref }}=1050{ }^{\circ} \mathrm{C}$ produces stress values that are experimentally observed in a typical SOI device [32].

For silicon nitride and a-Si thin films we use a TEC of $\alpha_{\mathrm{a}-\mathrm{Si}}=\alpha_{\mathrm{Si} 3 \mathrm{~N} 4}=3.6 \mu \mathrm{K}^{-1}$ which is the same as silicon's TEC and is within the reported range in Table 3.1. This choice of $\alpha_{\mathrm{Si3N} 4}$ results in very small thermal stress in the film $(<10 \mathrm{MPa})$. The film stress, then, is achieved by adding intrinsic stress to the $\mathrm{Si}_{3} \mathrm{~N}_{4}$ layer as an initial condition. This process allows us to make adjustments only to the initial stress of $\mathrm{Si}_{3} \mathrm{~N}_{4}$ layer when simulating structures with different stressing layers, while keeping all the other parameters constant. Unless otherwise stated, we use Young's modulus of $63 \mathrm{GPa}$ and Poisson ratio of 0.27 for the $\mathrm{Si}_{3} \mathrm{~N}_{4}$ and a-Si layers in all waveguides discussed in this thesis.

\subsubsection{Simulation considerations}


One of the most common tools for calculating mechanical stress and optical modes in a structure is Finite Element Method (FEM). In FEM, the problem is reduced to an eigenvalue equation. Then the system is divided into a large number of discrete mesh elements where the mesh density depends on the geometry of the domain. The overall solution of the entire domain is based on the eigen solutions to each individual mesh element. Both the stress/strain fields and optical modes can be calculated using FEM.

We use commercial FEM solver software Comsol ${ }^{\circledR}$ Multiphysics to solve the strain and modal distribution in the waveguide. Under proper boundary conditions Comsol ${ }^{\circledR}$ solves the static equilibrium equation that satisfies stress-strain relations, thermal effects and strain-displacement relations.

Schematic of the simulated structure is shown in Figure 3.4. Structure is assumed to be invariant along the propagation direction, $z$. The boundary conditions are chosen such that the system is pinned at the lower left corner $\left(x_{0}, y_{0}\right)$, the bottom right corner $\left(x_{1}, y_{0}\right)$ is free to move only in $x$ direction while the top, bottom, and the sides are surfaces that can freely move in $x$ - and $y$-directions. These boundary conditions force the solver to find a unique solution. In our calculations, we assume all the material layers, except for the straining layers, are at equilibrium (zero intrinsic stress) at $T_{r e f}$. Straining layers are given an initial stress at $T_{r e f}$ to account for intrinsic stress that results from the deposition process. 


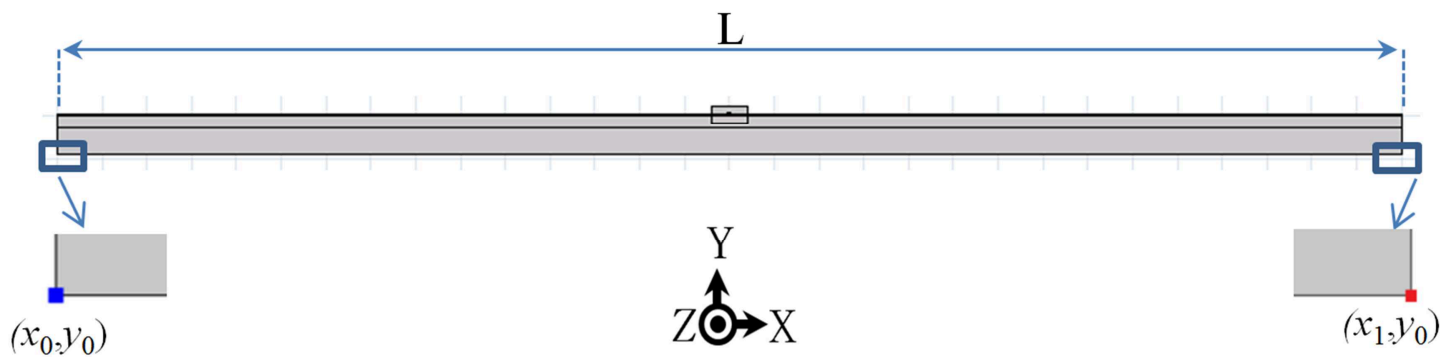

Figure 3.4 Structure modeled for mechanical stress-strain calculations.

Blue dot at $\left(x_{0}, y_{0}\right)$ is where the structure is 'pinned' both in $x$ and $y$ direction. Red dot at $\left(x_{1}, y_{0}\right)$ is pinned only in $y$ and is free in $x$ direction. Freedom in $x$ for red dot allows the wafer to bend. Length of the system was chosen to be $L=300 \mu \mathrm{m}$.

When stresses are present in a structure, the system deforms to reduce stress. In the SOI system the stress relief occurs through wafer bending and through elastic deformation from the edges and waveguide. Typically waveguides are situated far from the edges of the wafer and therefore the stress distribution inside the waveguides is not affected by the edge effect. It is critically important in simulations to choose a large length for the structure ( $L$ in Figure 3.4) such that the edge effects resulting from the boundary conditions do not affect the waveguide core region. By looking at the stress distribution in the straining layer at a given height, one can observe how far from the boundaries the core must be. Figure 3.5 demonstrates the edge effects exist up to about $25 \mu \mathrm{m}$ from the boundary. We chose length of the structure to be $300 \mu \mathrm{m}$. 


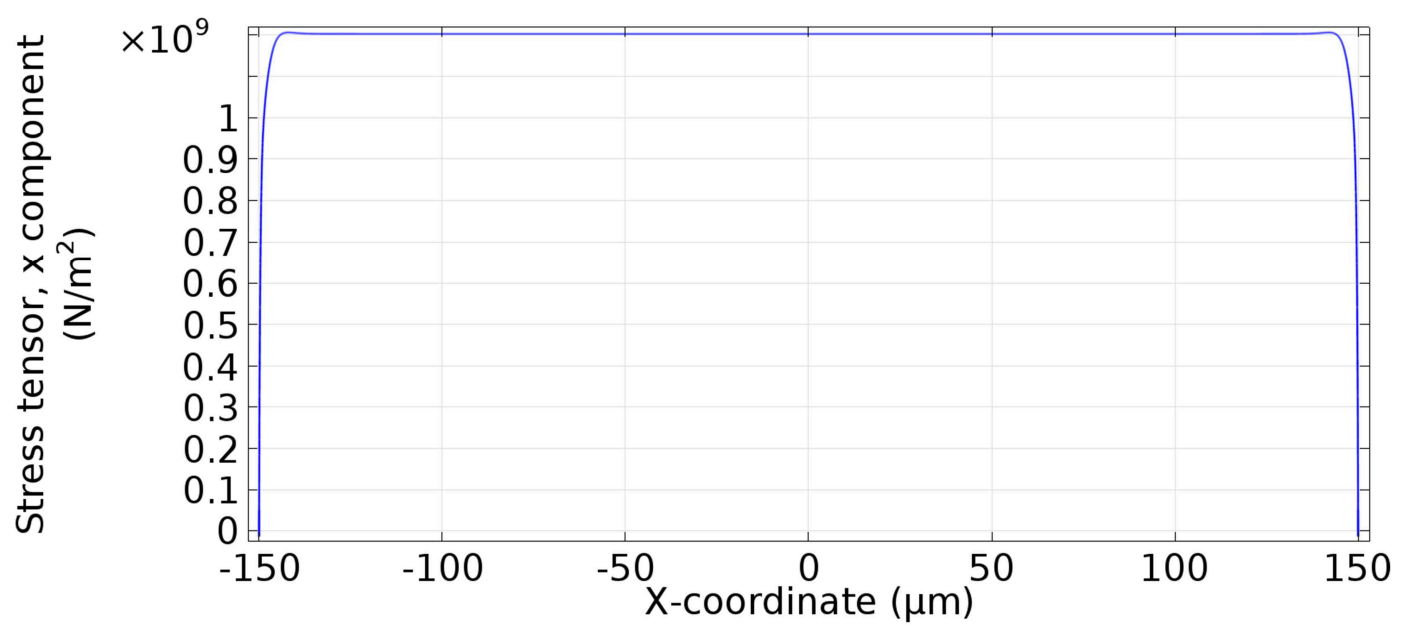

Figure 3.5 Stress distribution along $x$ at the mid-section of straining layer.

Sharp declines at the ends of the structure are artifacts of the boundary conditions imposed for numerical simulations.

In real systems the substrate is much larger than all the other layers. It is in order of $500 \times$ $500(\mu \mathrm{m})^{2}$. Simulating the entire structure that large with mesh size small enough to take into account the nanoscale features, requires extensive computing power and does not necessarily result in a more accurate solution. In order to make the mechanical calculation window smaller one can assign a large Young's modulus to the silicon substrate and use a much thinner substrate instead. It has been shown previously that using a Young's modulus of $130 \mathrm{TPa}\left(10^{3}\right.$ times higher than the typical $\left.E_{\mathrm{si}}\right)$ and a $5 \mu \mathrm{m}$ substrate, the resulting film stress varies by less than 5\% when compared to a system with $500 \mu \mathrm{m}$ thick substrate with $E_{\mathrm{si}}=130 \mathrm{GPa}$ [32].

\subsection{Stress modeling}

Here we show stress simulation results for the reported waveguides to demonstrate that the stress distribution in the modeled structure is a good match with the experimentally 
obtained stress distributions. Let's consider the $\mu \mathrm{m}$ scale waveguides examined by Cazzanelli et. al. in [14]. The waveguides were fabricated on $\sim 2.2 \mu \mathrm{m}$ thick SOI with a straining layer of silicon nitride $\left(\mathrm{Si}_{3} \mathrm{~N}_{4}\right)$. These waveguide produce second harmonic signals when excited by laser beam of $\lambda=2300 \mathrm{~nm}$. There are 3 types of waveguides studied; they are labeled SOI1, SOI2 and SOI3. SOI1 type waveguides are highly stressed by a $\mathrm{Si}_{3} \mathrm{~N}_{4}$ that has total tensile stress of $+1.2 \mathrm{GPa}$ and is $150 \mathrm{~nm}$ thick. SOI2 type waveguides are compressively stressed by a $500 \mathrm{~nm}$ thick $\mathrm{Si}_{3} \mathrm{~N}_{4}$ layer with -500 $\mathrm{MPa}$ stress. The stress layer in SOI3 waveguides is $500 \mathrm{~nm}$ and is stress compensated with $-60 \mathrm{MPa}$ stress. Unfortunately the origins of the stresses (thermal or intrinsic) and other $\mathrm{Si}_{3} \mathrm{~N}_{4}$ material properties are not provided; therefore we assume values from a range of reported values by other sources [31], [41]-[43], namely $\mathrm{E}_{\mathrm{Si} 3 \mathrm{~N} 4}=63 \mathrm{GPa}$ and $\alpha_{\mathrm{Si} 3 \mathrm{~N} 4}=3.6 \mu \mathrm{K}^{-1}$.

We start by simulating unpatterned silicon slabs. For a given set of material parameters a certain stress level is generated in various layers as shown in Figure 3.6. Since we chose $\alpha_{\mathrm{Si3N} 4}=3.6 \mu \mathrm{K}^{-1}$ there is almost no thermal stress in silicon nitride layer. We add initial stress equal to the measured stress of $\mathrm{Si}_{3} \mathrm{~N}_{4}$.
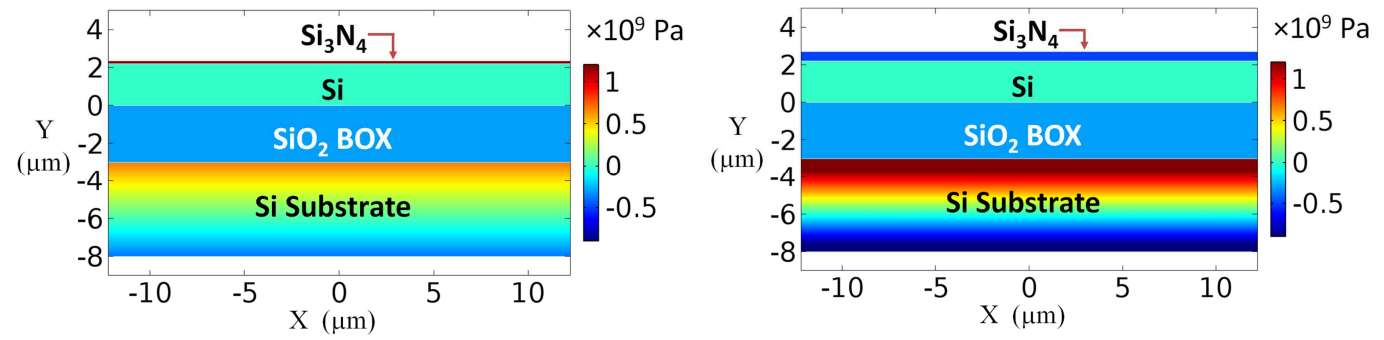

Figure 3.6 Stress component $\left(\sigma_{\mathrm{xx}}\right)$ distribution in SOI1 and SOI2 type slabs.

$\mathrm{Si}_{3} \mathrm{~N}_{4}$ straining later is $150 \mathrm{~nm}$ and $500 \mathrm{~nm}$ thick and has $+1.2 \mathrm{GPa}$ and $-500 \mathrm{MPa}$ total stress. 
The results of stress simulations for all three waveguide types are shown in Figure 3.7. In row (a) of Figure 3.7 exaggerated lattice deformation is shown schematically. In SOI1 since the top silicon nitride layer has tensile stress it causes compressive stress to the top of the core layer. The stress originating from the BOX (compressive) is much weaker and causes a small tensile stress at the bottom of the core. The situation is different for SOI2 and SOI3 since the top layer is compressively stressed. In SOI2 and SOI3 top and bottom of the core both have tensile stress but have different strengths, as indicated by the arrows. The simulation results showing an exaggerated $(\times 20)$ deformed shape of a waveguide as well as the strain component $\left(\varepsilon_{\mathrm{xx}}\right)$ are shown in row (b) of Figure 3.7 for all three types. Note the opposite direction of deformation of top of SOI1 and SOI2 cores.

Row (c) in Figure 3.7 shows the stress distribution in the core obtained by experimental measurements [14], [44]. The prefactor $\mathrm{C}=4.7 \pm 1.3 \mathrm{~Pa}^{-1}$ relates observed peak frequency shift in spectrum of $\mu$-Raman measurement to strain. Row (d) of Figure 3.7 shows the calculated stress maps for all three waveguides. Considering the micrometer spatial resolution of $\mu$-Raman spectroscopy, there is a good match between measured and simulated stress maps. 


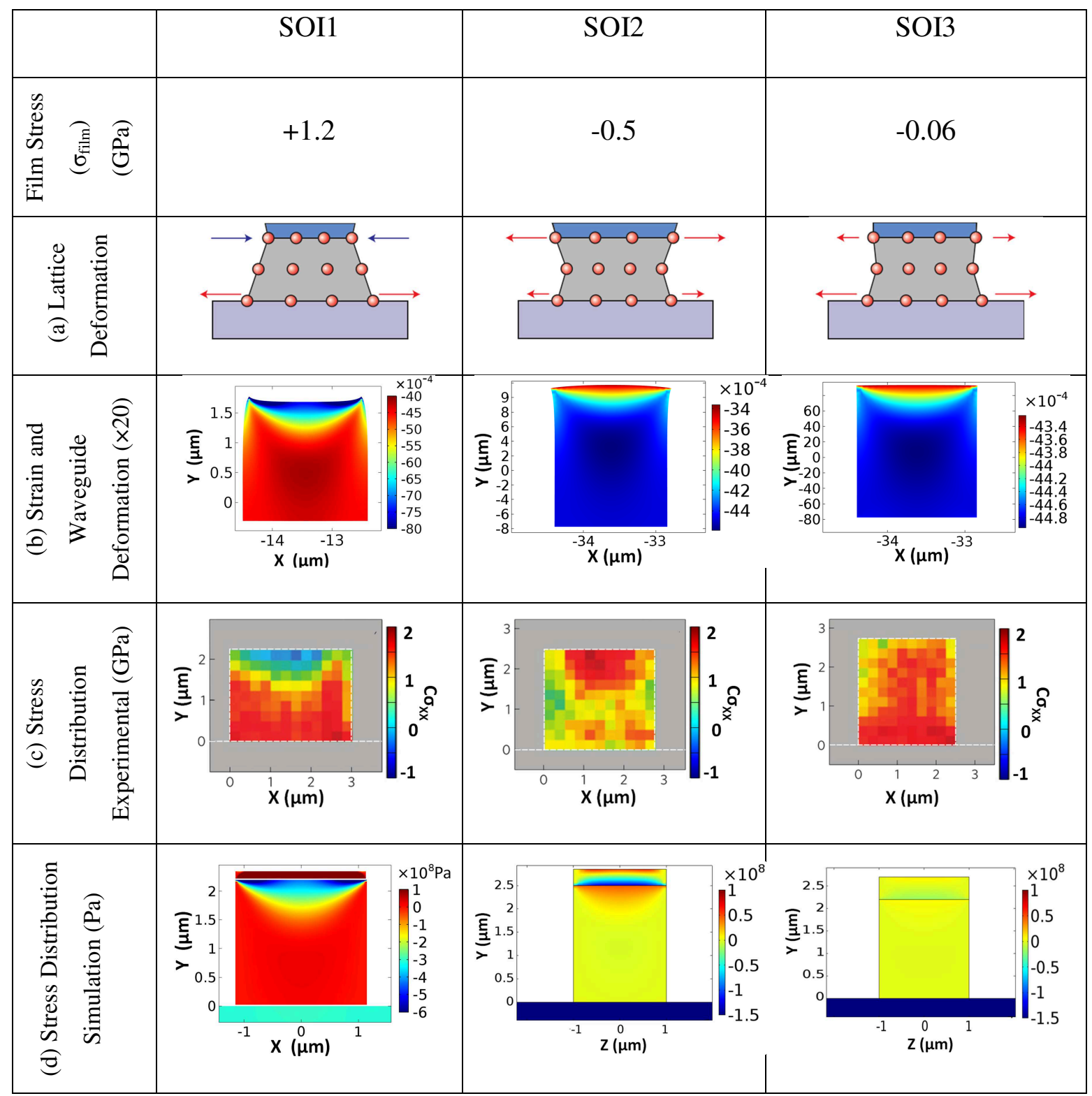

Figure 3.7 Stress and strain profiles for strained $\mu \mathrm{m}$ scale channel silicon waveguides.

(a) Exaggerated lattice deformation and direction of forces at top and bottom of waveguide. (b) Strain component $\left(\varepsilon_{\mathrm{Xx}}\right)$ distribution and the deformed shape of the waveguides scaled by factor of 20. (c) and (d)

Comparison between experimental and simulation results for stress component $\left(\sigma_{\mathrm{xx}}\right)$ distribution.

\subsection{Optical mode calculation}

Optical model calculations are also carried out by Comsol® Multiphysics. We only need to conduct the optical calculations in a small area in the vicinity of the waveguide, as 
opposed to the entire mechanical calculation window. Figure 3.8 shows the optical calculation window chosen for the $\mu \mathrm{m}$ scale waveguides $(48 \mu \mathrm{m} \times 7.5 \mu \mathrm{m})$. The window is chosen to be large enough so that the optical mode in the waveguide does not interact substantially with the boundaries. The optical mode calculation windows size for the nanometer scale waveguides is $8 \mu \mathrm{m} \times 4 \mu \mathrm{m}$.

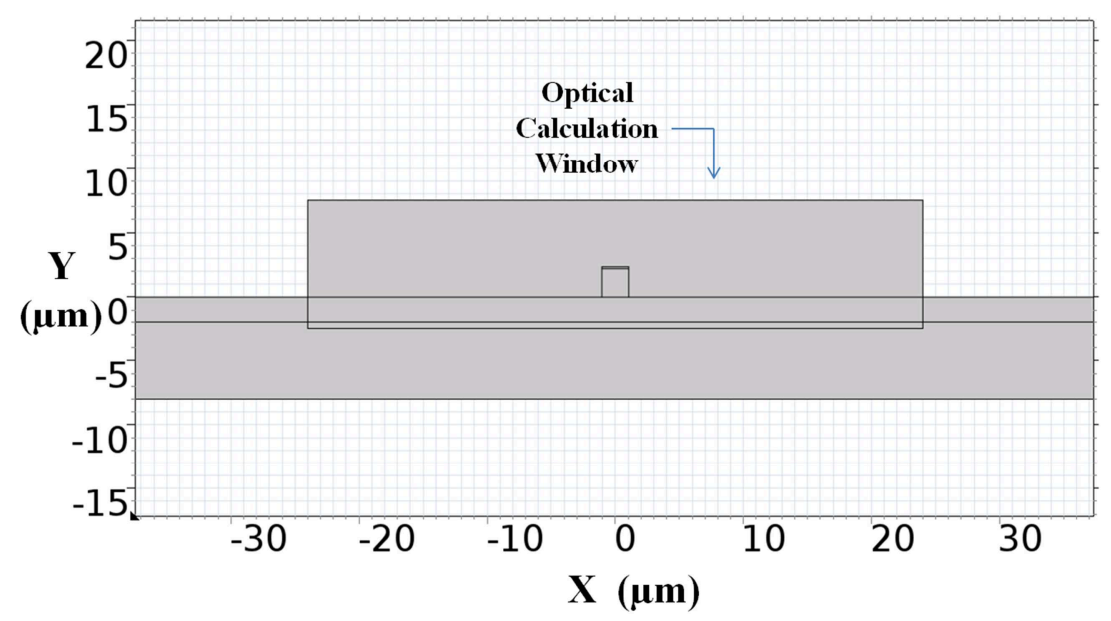

Figure 3.8 Optical calculation windows for the $\mu \mathrm{m}$ scale channel waveguides.

In order to find a solution for the optical modes a seed value for the effective index $\left(\mathrm{n}_{\mathrm{eff}}\right)$ of the desired mode needs to be supplied to the software. The software then seeks eigen solutions to the wave equations using Finite Element Method. A predetermined number of solutions that satisfy the equations and boundary conditions are calculated which include both quasi TE and quasi TM modes. Some of the calculated mode solutions might not be physical and are the artifacts of the numerical method used. One needs to manually select the correct desired mode, typically the fundamental mode which has the highest $\mathrm{n}_{\text {eff. }}$. 


\subsection{Stress-optic effect}

Stress-optic (or photoelastic) effect is the change in refractive index of material as a function of mechanical stress. This relationship can be described as follows [45]:

$$
\begin{aligned}
& \Delta n_{x}=n_{x}-n_{0}=-C_{1} \sigma_{x}-C_{2}\left(\sigma_{y}+\sigma_{z}\right) \\
& \Delta n_{y}=n_{y}-n_{0}=-C_{1} \sigma_{x}-C_{2}\left(\sigma_{x}+\sigma_{z}\right)
\end{aligned}
$$

where, $\sigma_{\mathrm{x}}, \sigma_{\mathrm{y}}$ and $\sigma_{\mathrm{z}}$ and are the principal stress tensor components along the $x, y$ and $z$ axis directions, respectively; $n_{0}$ is the refractive index of the material without stress; $C_{1}$ and $C_{2}$ are the stress-optical constants.

Stress is first calculated for the modeled SOI waveguide system. Then, the resulting stress distribution is used in equations (3.5) and (3.6) to modify the refractive index distribution for the optical mode calculations. 


\section{Chapter: Stress/Strain Characterization Using Figure of Merit}

In order to induce second order nonlinearity in a silicon crystal the symmetry needs to be broken by application of anisotropic stress. A homogeneous strain does not break the central symmetry of the crystalline silicon; it is the inhomogeneous strain (i.e. a strain gradient) which induces a distortion in the crystal symmetry and therefore a non-zero $\chi_{\mathrm{Si}^{(2)}}{ }^{2}$ [9], [13], [14], [44]. The strain gradient varies in the waveguide cross-section. It is of critical importance for regions of high strain gradient to overlap with the optical mode. In this chapter we first analyze the spatial distribution of strain gradient and optical mode for various waveguide structures. We then introduce a Figure of Merit (FOM) in order to quantitatively compare the performance of various waveguide designs. Finally we use the proposed FOM to optimize the waveguide design to reach maximum nonlinearity.

\subsection{Analysis of the strain induced Pockels effect in silicon waveguides}

There are four sets of experimental data available to date for stress-induced $\chi_{\mathrm{Si}}{ }^{(2)}$ in $\mathrm{Si}$ waveguides measured by Pockels effect [8], [9], [13] or second harmonic generation [14]. We analyze the strain distribution of the micrometer-scale channel waveguide on a $2 \mu \mathrm{m}$ SOI in [14], and the nanowire rib waveguide on a $220 \mathrm{~nm} \mathrm{SOI}$ in [9] and [13]. The waveguides in [9] and [13] are only different in width. The results in [8] cannot be utilized since the reported geometrical parameters are not sufficiently detailed. We first use a finite element method software COMSOL ${ }^{\circledR}$ Multiphysics to simulate the induced strain and modal fields for these two structures.

\subsubsection{Micro-scale channel waveguide}

For the SOI1 channel waveguide in [14], the straining layer is $150 \mathrm{~nm}$ and has $+1.2 \mathrm{GPa}$ stress. The $\mathrm{Si}_{3} \mathrm{~N}_{4}$ layer is deposited exclusively on top of the waveguide core with the 
main source of strain inhomogeneity located at the top cladding/core interface. Figure 4.1 $(a, b, c)$ illustrates the strain $\varepsilon_{x x}$, the deformed shape of the waveguide core, and strain gradient $\partial \varepsilon_{\mathrm{xx}} / \partial y$, respectively, in a $2.3 \mu \mathrm{m}$ wide channel waveguide. The strength of the induced $\chi^{(2)}$ on the optical mode depends on the strain gradient reaching from the top to the central region of the waveguide core, where the optical mode intensity is maximal, as seen in Figure 4.1 (d). In this comparatively large waveguide $(2.3 \mu \mathrm{m} \times 2 \mu \mathrm{m}$ crosssection) the confinement factor is nearly $100 \%$. Figure $4.1(\mathrm{e})$ shows the overlap of this strain gradient and the optical mode distribution that represents the spatial distribution of second order nonlinearity $\chi_{\mathrm{Si}}{ }^{(2)}$, as explained in more detail in section 4.2. Experimentally, using this design an effective $\chi^{(2)}=20 \pm 10 \mathrm{pm} / \mathrm{V}$ was achieved. 


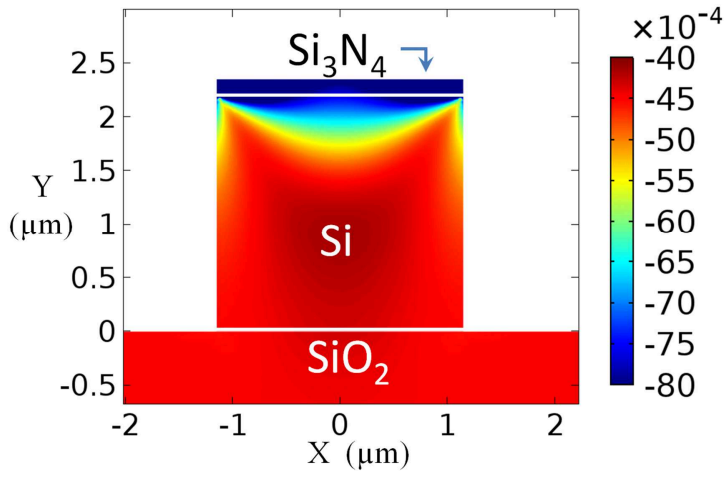

(a)

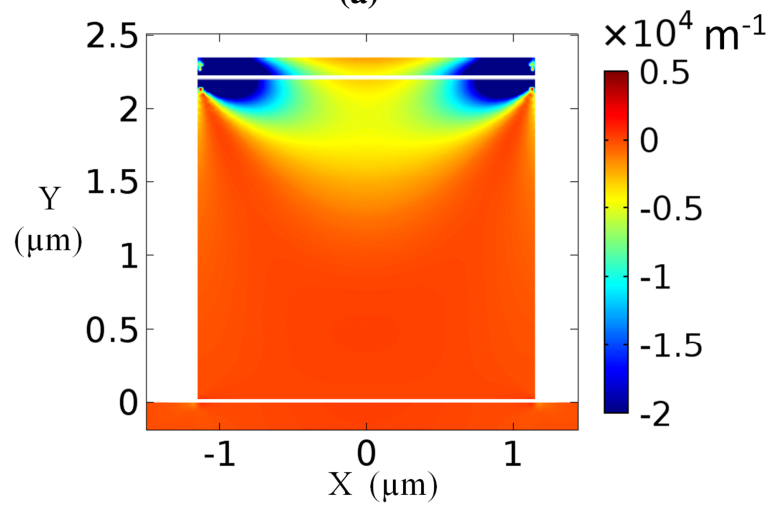

(c)

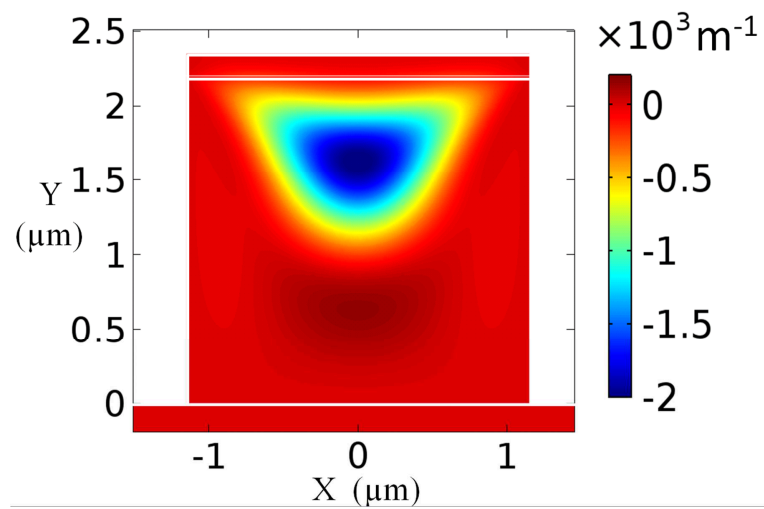

(c)

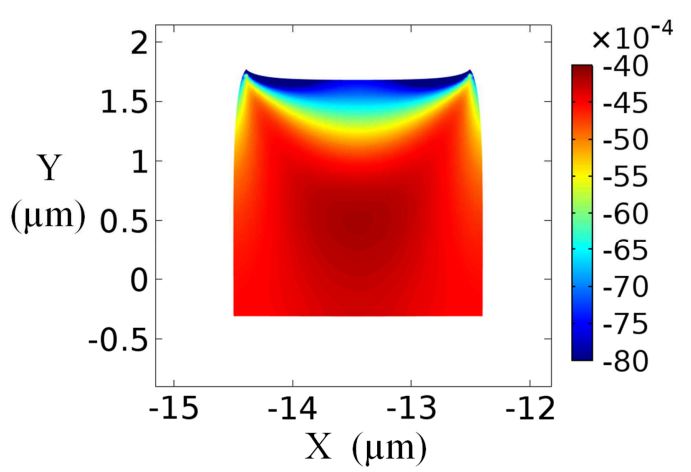

(b)

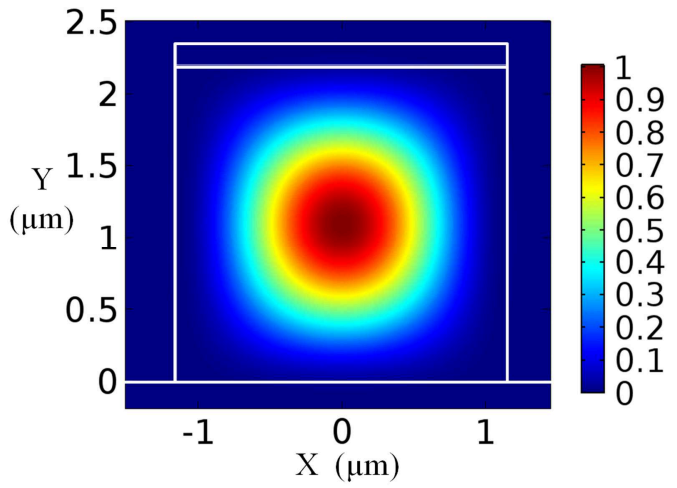

(d)

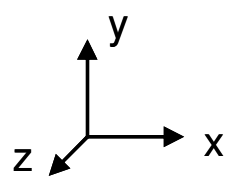

Figure 4.1 Strain-induced effects for a $2.3 \mu \mathrm{m}$ wide channel waveguide in $2 \mu \mathrm{m}$ thick SOI.

The $\mathrm{Si}_{3} \mathrm{~N}_{4}$ cladding layer is $150 \mathrm{~nm}$ thick and has $+1.2 \mathrm{GPa}$ stress. (a) Strain distribution $\varepsilon_{\mathrm{xx}}$; (b) Strain distribution $\varepsilon_{\mathrm{xx}}$ with deformed shape of the waveguide core, deformation is scaled by a factor of 20; (c) Strain gradient $\partial \varepsilon_{\mathrm{xx}} / \partial y$; (d) Waveguide fundamental TE mode distribution; (e) Overlap integral of $\partial \varepsilon_{\mathrm{xx}} / \partial \mathrm{y}$ and the modal field, representing the spatial distribution of the second-order nonlinearity. 


\subsubsection{Nano-scale ridge waveguide}

In the nanowire rib structure reported in [9], the waveguide cross-section is substantially smaller, between $300 \mathrm{~nm}$ to $500 \mathrm{~nm}$ in a $220 \mathrm{~nm}$ SOI wafer. The straining layer which is $350 \mathrm{~nm}$ thick and has $+1 \mathrm{GPa}$ stress, blankets both the top and the sidewalls of the waveguide core. Figure 4.2 (a) shows an Scanning Electron Microscope (SEM) image of the waveguide [13].

A major challenge in simulating this structure is that the straining layer is not uniform around the waveguide. There is a gap near the sidewalls of the core in the $\mathrm{Si}_{3} \mathrm{~N}_{4}$ layer. Formation of these gaps is a stress relief mechanism in highly stressed films; therefore we expect the local mechanical properties around the gap layer to be different from other parts of the film. In the literature there is no information on how various mechanical properties change because of these gaps. In addition, the exact depth, angle and location of the gap are not reported. Figure 4.2 (b) shows the simulated structure that has additional parameters for the gap. As a first order approximation we assume the mechanical properties of $\mathrm{Si}_{3} \mathrm{~N}_{4}$ layer are uniform and the position of the gap is directly above the core's vertical walls and gap_y $=60 \mathrm{~nm}$. Later in section 4.6 we will investigate how changing position of the gap influences the obtained results.

The calculated stress distribution is shown in Figure 4.2 (c). The strain component $\varepsilon_{\mathrm{xx}}$ and the deformed shape of the waveguide core (scaled $\times 20$ ) are illustrated in Figure $4.2(\mathrm{~d})$. The nano-scale rib geometry compared to the micro-meter channel waveguide discussed in section 4.1.1 allows higher strain inhomogeneity since the cross-section is much smaller and the bottom of the waveguide is pinned to the lateral silicon slab, while the top is free to deform. 


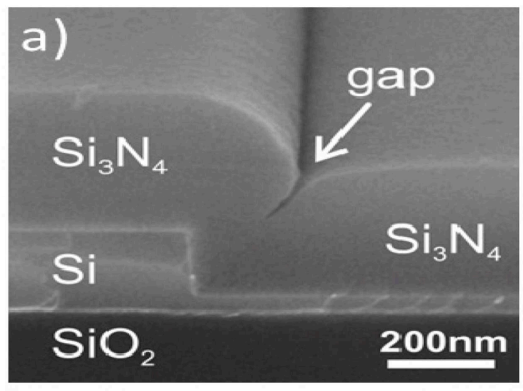

(a)

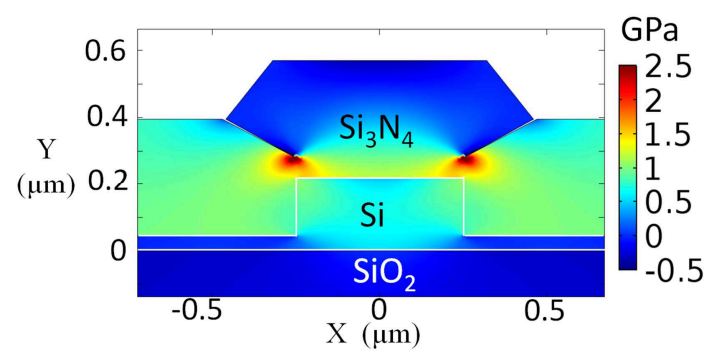

(c)

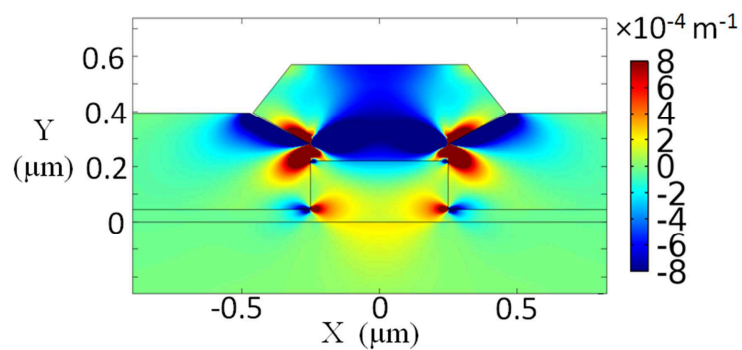

(e)

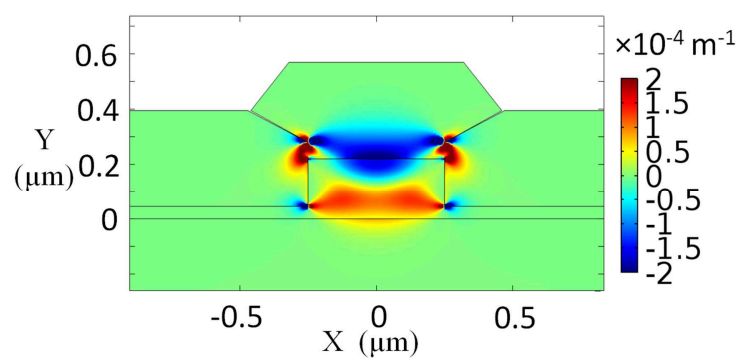

(g)

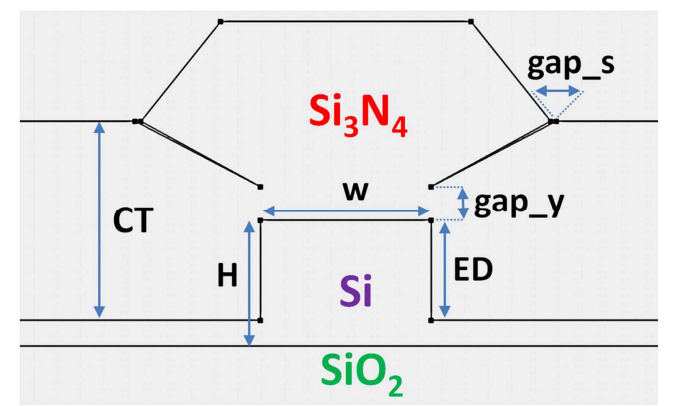

(b)

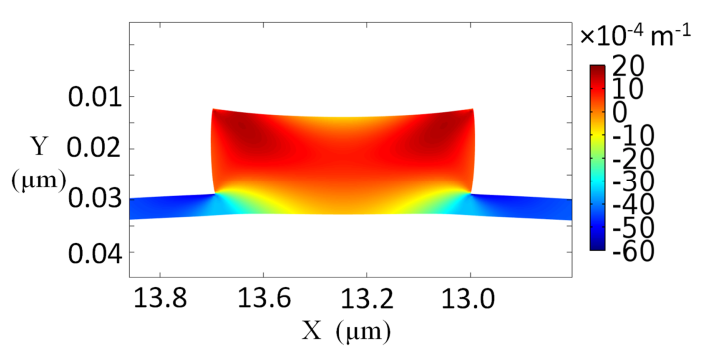

(d)

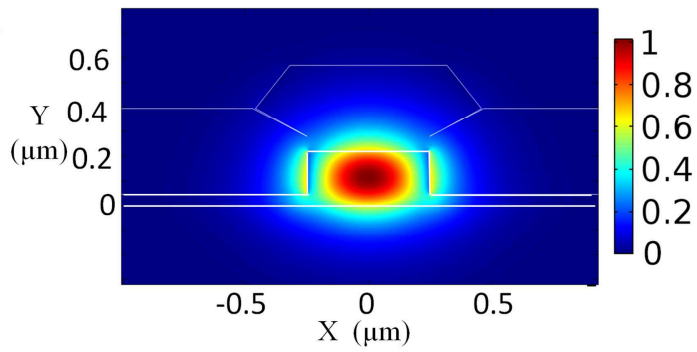

(f)

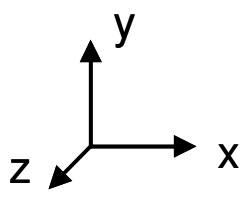

Figure 4.2 Strain induced effects for a $500 \mathrm{~nm}$ wide ridge waveguide in $220 \mathrm{~nm}$ SOI. The $350 \mathrm{~nm}$ thick $\mathrm{Si}_{3} \mathrm{~N}_{4}$ layer has gaps near waveguide sidewalls. Film stress $\sigma_{\text {film }}=+1 \mathrm{GPa}$.

(a) Scanning Electron Microscope (SEM) image of a nano scale ridge waveguide [13]. (b) Schematic image of the simulated waveguide structure. Here $y$ spacing between the gap and top of the waveguide gap_y $=60 \mathrm{~nm}$, gap width at the top gap_s $=10 \mathrm{~nm}$ and gap opening angle is $\sim 1^{\circ}$. (c) Stress component $\sigma_{\mathrm{xx}}$ profile. (d) Strain component $\varepsilon_{\mathrm{xx}}$ profile with deformed shape of the waveguide $(\times 20)$. (e) Strain gradient $\partial \varepsilon_{\mathrm{xx}} / \partial \mathrm{y}$. (f) Normalized mode intensity profile. (g) Overlap of strain gradient and mode profile. 
The strain gradient $\partial \varepsilon_{\mathrm{xx}} / \partial y$ map, shown in Figure 4.2 (e), indicates there are two main regions with strong strain inhomogeneity inside the waveguide: the bottom corners of the sidewalls and the top of waveguide near the core/cladding interface. Note the different signs of strain gradient for these regions. Outside the waveguide gaps in the cladding are strong sources of strain inhomogeneity that changes the strain distribution inside the waveguide. The existence of high strain inhomogeneity near side walls was observed by the micro-Raman and THz mappings experiments [9] as well. However, due to micrometer scale resolution of these techniques, the change in sign of strain gradient across the nano-rib waveguide cannot be observed. Hence, calculated strain gradient maps provide details that are not previously reported.

Figure 4.2 (f) shows the distribution for the fundamental TE mode. The mode overlap with the strain gradient is shown in Figure $4.2(\mathrm{~g})$. The overlap shows the how much the mode is affected by the induced $\chi_{\mathrm{Si}}{ }^{(2)}$. Because of the small size of the waveguide the optical confinement factor is only $66 \%$ (for $500 \mathrm{~nm}$ wide core), with a significant fraction of the mode distribution outside of the Si core. Indeed, the part of the waveguide mode outside the $\mathrm{Si}$ core is not affected by the induced $\chi_{\mathrm{Si}}{ }^{(2)}$. We also observe that the sign difference of strain gradient between the top and bottom of the core limits the effective strain gradient experienced by the mode. The highest $\chi_{\mathrm{Si}}{ }^{(2)}$ achieved using this geometry is for a $300 \mathrm{~nm}$ wide waveguide that yields $\chi_{\mathrm{xxy}}^{(2)}=190 \mathrm{pm} / \mathrm{V}$.

\subsection{Figure of Merit}

So far in the literature comparisons between different waveguide geometries have been conducted only experimentally. Quantitative comparison between different waveguide geometries in design stage has not been conducted, due to a lack of suitable design tools. 
A rigorous estimation of $\chi^{(2)}$ induced by the strain inhomogeneity is intricate and requires complex ab-initio calculations [9], which are not practical for use as a design engineering tool to optimize waveguide geometry and straining layer parameters. In addition discrepancies between theoretically calculated values of $\chi^{(2)}$ and observed experimental results have been reported [10]. Most theoretical models predict values of $<0.05 \mathrm{pm} / \mathrm{V}$ for a typical $1 \mathrm{GPa}$ stress induced $\chi^{(2)}$ in silicon [10], [28]. However, experimentally values in scale of tens of $\mathrm{pm} / \mathrm{V}$ have been observed in several studies [7], [8], [14] with the highest reported value being $190 \mathrm{pm} / \mathrm{V}$ [13]. The discrepancy between theory and experiments might point to missing physics in current models [10]. To circumvent these difficulties and simplify evaluation of the induced Pockels effect, we introduce a Figure of Merit (FOM) based on experimentally observed results and a recent phenomenological study [11]. A simplified 2D centro-symmetric lattice model discussed in section 2.4 and [10] shows that strain gradient $\partial \varepsilon_{\mathrm{xx}} / \partial y$ produces a net electric field in $y$ direction parallel to the electric modulation field. Although using this model the estimated $\chi^{(2)}$ is far smaller than what experiments suggest, it is reasonable to assume that the $\partial \varepsilon_{\mathrm{xx}} / \partial y$ term is the main source of $\chi^{(2)}$ [13]. Because of lack of strain symmetry in the vertical direction in the waveguide (see Figure 4.1 (a) and Figure 4.2 (d)), the strain gradient along the $y$ direction is the dominant factor for inducing non-centro-symmetry, consistent with the findings in [9], [11], [13], [14]. A recent phenomenological study suggests $\chi_{x x y}^{(2)}$, related to quasi-transverse-electric (TE) mode with electric modulation field in $y$ direction, is directly proportional to strain gradient $\partial \varepsilon_{x x} / \partial y$ by a scaling coefficient $C_{x x y}[11]$ :

$$
\chi_{x x y}^{(2)}=C_{x x y}\left(\frac{\partial \varepsilon_{x x}}{\partial y}\right)
$$

For quasi-transverse-magnetic (TM) mode equation (4.1) needs to be modified to: 


$$
\chi_{y y y}^{(2)}=C_{y y y}\left(\frac{\partial \varepsilon_{x x}}{\partial y}\right)
$$

Since the strain gradient profile is not uniform in the waveguide cross-section we require that it overlaps with the optical mode. Therefore, we define a Figure of Merit (FOM) as the overlap integral of the strain gradient $\partial \varepsilon_{\mathrm{xx}} / \partial y$ and the normalized mode intensity distribution in transverse plane $(x, y)$ :

$$
F O M=\frac{\iint_{S i} \frac{\partial \varepsilon_{x x}}{\partial y}|E|^{2} d x d y}{\int_{-\infty}^{\infty} \int_{-\infty}^{\infty}|E|^{2} d x d y}
$$

where $E$ is the electric field distribution of the waveguide fundamental mode and $\varepsilon_{\mathrm{xx}}$ is the strain component along the $x$ direction. As defined, FOM is the average of the strain gradient distribution weighted by the mode field intensity and as such it has a unit of $\mathrm{m}^{-1}$. This approach is similar to how the effective index of a mode, representing the spatial average of the refractive index experienced by the mode, is computationally calculated. The integration in the numerator only encompasses the silicon region, since that's the only active part of the waveguide. The portion of the mode outside the waveguide does not contribute to effective $\chi^{(2)}$.

In our analysis only quasi-TE (TE) mode is considered since for $220 \mathrm{~nm}$ SOI waveguides quasi-TM mode is mainly guided outside the silicon core which is less desirable from confinement point of view. In addition experimental results used as our references only include TE mode experiments.

We calculate the FOM for the two types of structures reported in [9] and [13], assuming the stress in the bottom oxide layer of -300 $\mathrm{MPa}$, a typical value for thermally grown $\mathrm{SiO}_{2}$. There are three types of waveguide in [9] each with different stress levels, as 
detailed in Figure 3.7. Waveguides in [13] feature $1 \mathrm{GPa}$ tensile in $\mathrm{Si}_{3} \mathrm{~N}_{4}$ stress layers. The calculated FOM is shown in Figure 4.3 and are correlated with the experimental $\chi^{(2)}$ values, with a correlation coefficient $r=0.95$. The high correlation indicates that the introduced FOM is a good measure of the strength of strain-induced Pockels effect in silicon waveguides. Note that the $\chi^{(2)}$ values are corrected using the waveguide confinement factor to represent the effective $\chi_{\text {xxy }}^{(2)}$ for the waveguide mode (effective $\chi^{(2)}$ $=$ waveguide confinement factor $\left.\times \chi_{\mathrm{Si}}^{(2)}\right)$ to compensate for the evanescent tail of the mode which propagates outside the silicon core. For the $(300 \mathrm{~nm} \times 220 \mathrm{~nm})$ ridge waveguide the confinement factor is $\sim 42 \%$ and $\chi_{\mathrm{Si}^{(2)}}{ }^{2}=190 \mathrm{pm} / \mathrm{V}$ resulting in effective $\chi^{(2)}=79.8 \mathrm{pm} / \mathrm{V}$. Details of geometrical and mechanical parameters of all points in Figure 4.3 are available in Appendix A .

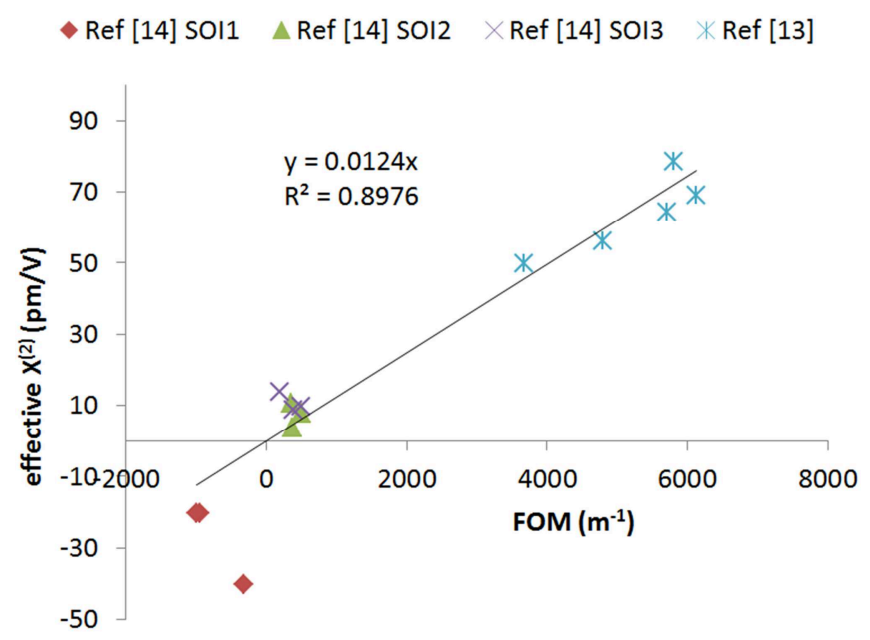

Figure 4.3 Correlation between experimental second order nonlinearity $\chi^{(2)}$ values reported in [13], [14] and the calculated Figure of Merit (FOM).

Note that effective $\chi^{(2)}=$ confinement factor $\times \chi_{\mathrm{Si}}^{(2)}$.

From the correlation plot we can deduce that

$$
\text { Effective } \chi^{(2)} \approx C \cdot F O M
$$


where $\mathrm{C}$ is the proportionality constant, which is equal to $0.0124 \mathrm{pm}^{2} / \mathrm{V}$. Figure 4.4 shows how FOM and effective $\chi^{(2)}$ change as width varies. FOM values are calculated and effective $\chi^{(2)}$ are experimental results reported in [13].

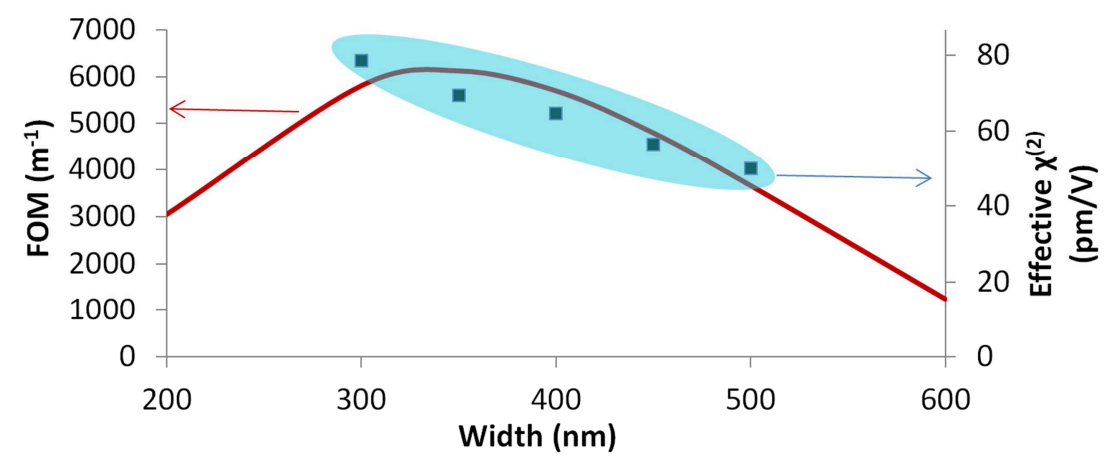

Figure 4.4 Figure of merit and effective $\chi^{(2)}$ as functions of waveguide width.

Solid red line is the calculated FOM and blue squares are experimentally observed effective $\chi^{(2)}$ [13].

\subsection{Geometric optimization}

We now choose the nano-scale rib waveguide design with the highest reported $\chi_{\mathrm{Si}}{ }^{(2)}=190$ $\mathrm{pm} / \mathrm{V}$ (effective $\chi^{(2)}=79.8 \mathrm{pm} / \mathrm{V}$ ) and calculate $\mathrm{FOM}$ of $5810 \mathrm{~m}^{-1}$, as the starting point for further optimization. Using the methodology outlined above we investigate the effects of design parameters of a nanowire rib waveguide on the device performance, i.e. effective $\chi^{(2)}$. Assuming a core height of $220 \mathrm{~nm}$, width of $300 \mathrm{~nm}$, cladding thickness of $350 \mathrm{~nm}$ and etch depth of $175 \mathrm{~nm}$, as in [9] and [13], we scan different parameters and obtain highest possible FOM and effective $\chi^{(2)}$. The optimization process is carried out in for structures with gaps in the cladding as seen in previous section and also for simple uniform cladding.

\subsubsection{Optimization of nano-scale waveguide with gaps in cladding}

For the nano-rib waveguide, detailed in Figure 4.2 and section 4.1.2, a multi-parameter optimization procedure yielded the following waveguide geometry to achieve the 
maximum value for the FOM: width $=350 \mathrm{~nm}$, etch depth $=100 \mathrm{~nm}$ and height $=220$ $\mathrm{nm}$. Here we assumed the location of the endpoints of the gaps are in constant in relation to the core, i.e. gap_y $=60 \mathrm{~nm}$. The rationale behind choosing this geometry is explained in detail in section 4.6. Changing the cladding thickness requires appropriate adjustment of the geometry and location of the gaps. As these adjustments are highly dependent on exact deposition condition and not known, we assumed the cladding thickness to be constant.

Change in FOM and effective $\chi^{(2)}$ as a function of width (W), etch depth (ED) and height (H) is illustrated in Figure 4.5 (a), (b) and (c), respectively. Strain gradient and mode overlap map of the optimized waveguide can be seen in Figure 4.5 (d). For each parametric scan, all other parameters are held constant at the optimized value.

Decreasing the waveguide width from $700 \mathrm{~nm}$ to $\sim 350 \mathrm{~nm}$ increases the FOM since bottom of the sidewalls, which have high gradient, progressively overlap more with the optical mode. Below $\sim 350 \mathrm{~nm}$ width the decrease in confinement dominates the FOM and it decreases. Scanning the etch depth shows that highest FOM can be achieved when the waveguide is shallowly etched $\sim 100 \mathrm{~nm}$. Etching more than or less than $\sim 100 \mathrm{~nm}$ moves the bottom of the sidewalls away from the modes center.

Decreasing the height below $220 \mathrm{~nm}$, results in more overlap between the top core/cladding interface and the mode. The sign of strain gradient at the top is opposite of the bottom of the waveguide, therefore FOM decreases for height $<220 \mathrm{~nm}$. Height larger than $220 \mathrm{~nm}$ moves the center of the mode above the bottom corners and decreases FOM. Indeed, $220 \mathrm{~nm}$ SOI is the optimum height to obtain strain induced $\chi^{(2)}$. 


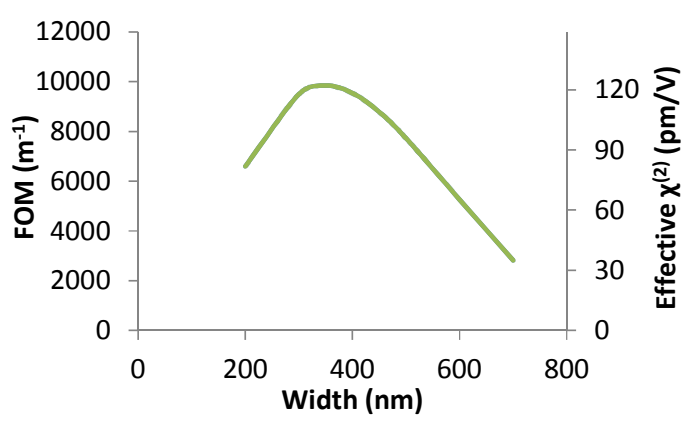

(a)

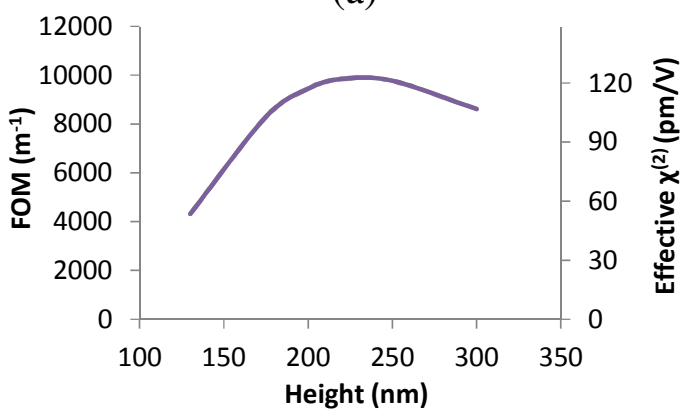

(c)

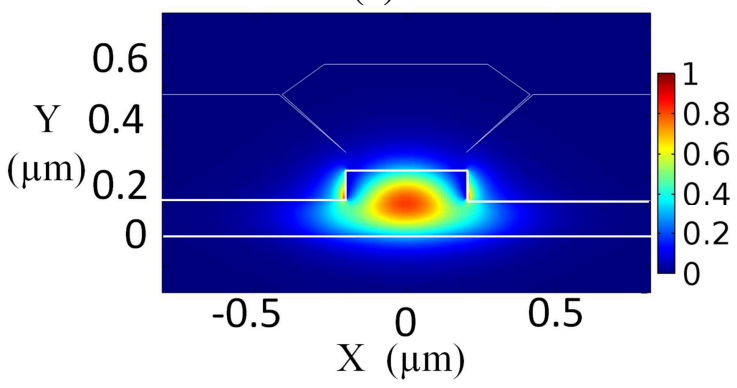

(e)

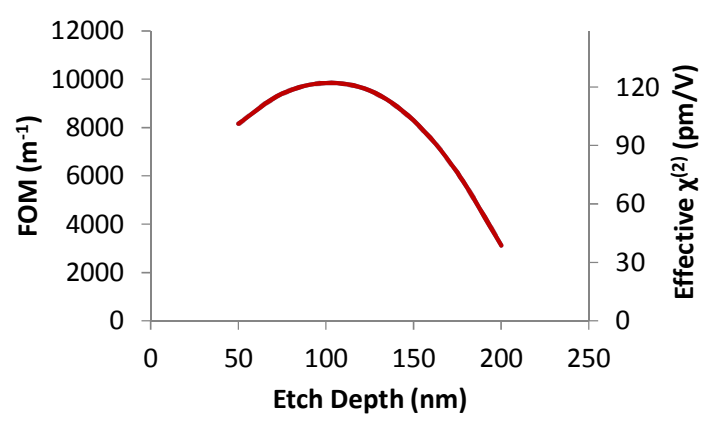

(b)

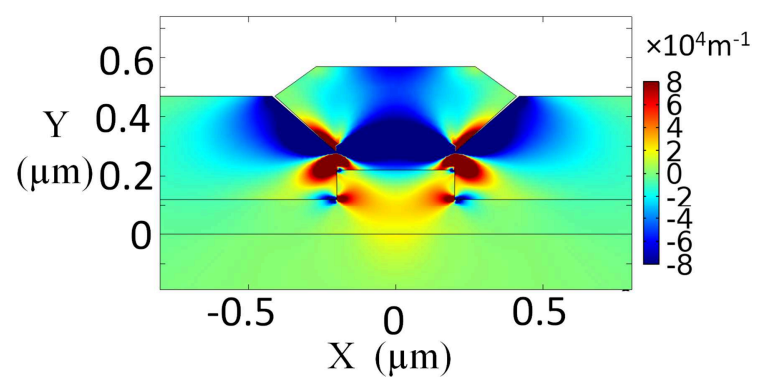

(d)

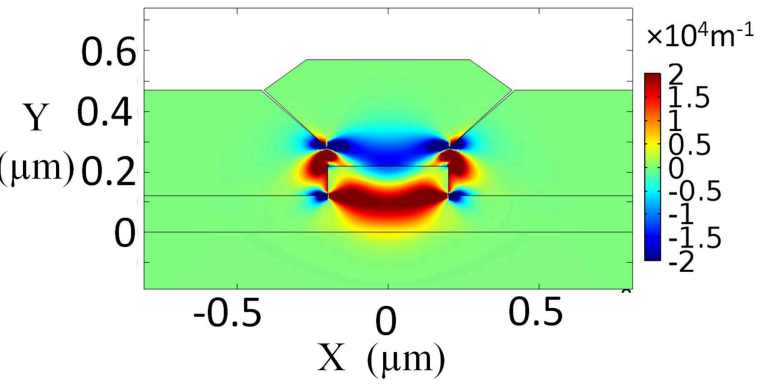

(f)

Figure 4.5 Optimization of strain induced effects in a nano scale rib waveguide with cracks in cladding layer.

Change in FOM and effective $\chi^{(2)}$ as a function of (a) width, (b) etch depth (ED), and (c) height (h). (d), (e) and (f) show strain gradient, normalized optical mode intensity, and overlap of strain gradient and normalized optical mode intensity for the optimized geometry: width $=350 \mathrm{~nm}$, etch depth $=100 \mathrm{~nm}$,

$$
\text { height }=220 \mathrm{~nm} \text {, resulting in } \mathrm{FOM}=9800 \mathrm{~m}^{-1} \text {. }
$$




\subsubsection{Optimization of nano-scale waveguide with uniform cladding}

So far we have modeled the structure with gaps in the cladding layer as they were observed in the experiment reported in [13], in order to provide a more realistic model of the actual structure that was investigated there. However, inclusion of gaps in the cladding introduces a number of modeling parameters which are not known, such as the endpoints, angles and widths of the gaps as well as mechanical parameters of $\mathrm{Si}_{3} \mathrm{~N}_{4}$ around the gaps leading to increased uncertainty in the optimization results. Such gaps are not always observed in stressing cladding layers. Here we analyze a more generic waveguide structure where the cladding layer deposition covers all the top and ridge of the waveguide uniformly, and that does not include gaps in the cladding layer. The waveguide geometry and the stress distribution for such a waveguide are shown in Figure 4.6 (a). Strain map and the deformed shape of the waveguide are illustrated in Figure 4.6 (b). Strain gradient inside the waveguide core can be seen in Figure 4.6 (c). Note the sign difference of the strain gradient between the top and bottom of the waveguide, which is similar to the case with gap in the cladding. Fundamental TE mode distribution and its overlap with the strain gradient are shown in Figure 4.6 (d) and (e), respectively. 


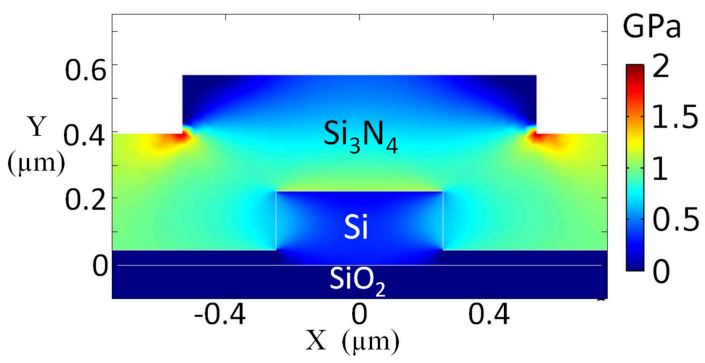

(a)

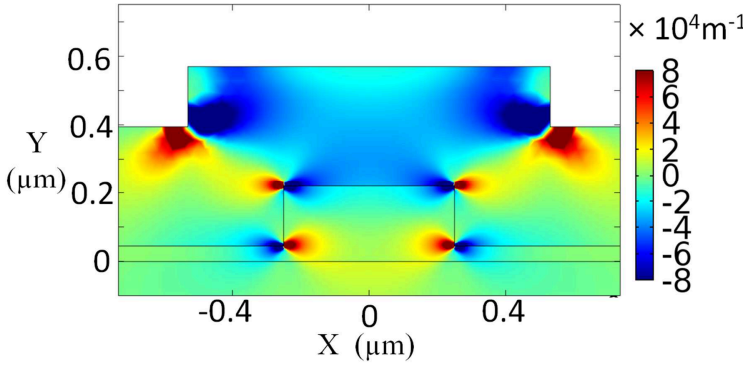

(c)

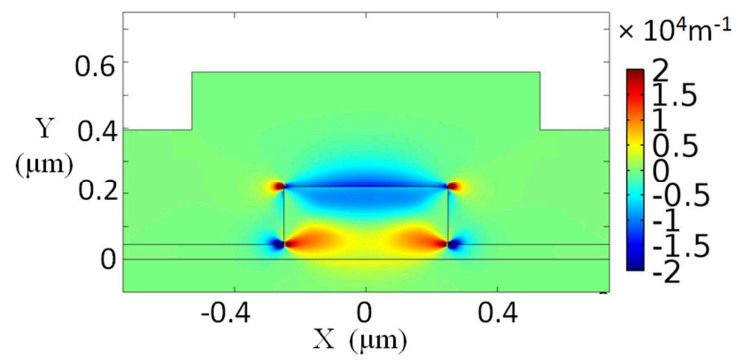

(e)

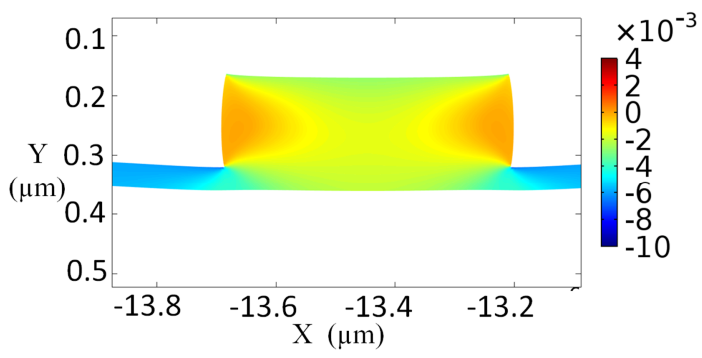

(b)

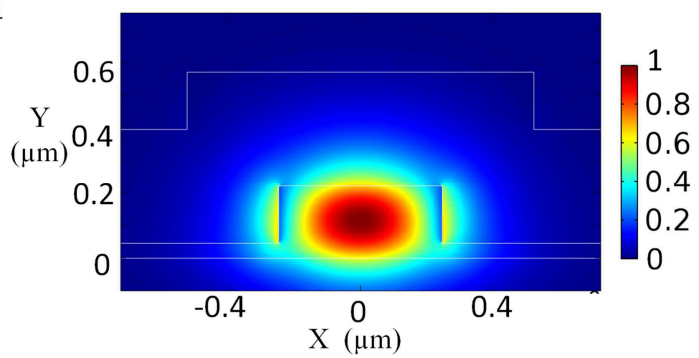

(d)

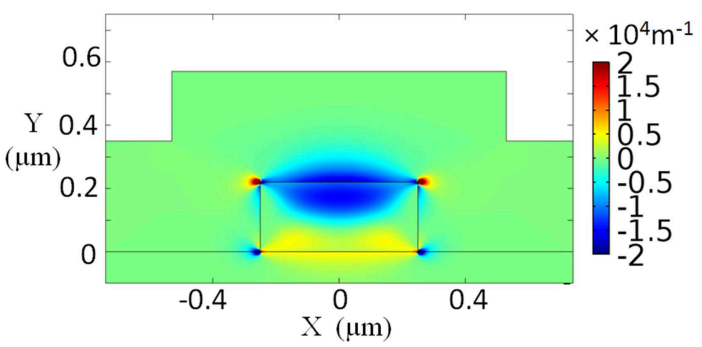

(f)

Figure 4.6 Strain induced effects for a $500 \mathrm{~nm}$ wide ridge waveguide in $220 \mathrm{~nm}$ SOI. The $350 \mathrm{~nm}$ thick $\mathrm{Si}_{3} \mathrm{~N}_{4}$ layer covers waveguide sidewalls uniformly. Film stress $\sigma_{\text {film }}=+1 \mathrm{GPa}$.

(a) Stress distribution $\sigma_{x x}$; (b) Strain distribution $\varepsilon_{x x}$ with deformed shape of the waveguide core, scaled by $\times 20$; (c) Strain gradient $\partial \varepsilon_{\mathrm{xx}} / \partial \mathrm{y}$; (d) Waveguide fundamental TE mode distribution; (e) Overlap of $\partial \varepsilon_{\mathrm{xx}} / \partial \mathrm{y}$ and the modal field. (f) Overlap of $\partial \varepsilon_{\mathrm{xx}} / \partial \mathrm{y}$ and the modal field for a fully etched (channel) waveguide. In this geometry FOM is dominated by negative values near the top interface of the core. The subtraction of FOM due to the bottom gradient is smaller for the channel structure, compared to rib geometry.

It can be seen in Figure 4.6 (e) that the main regions of the strain inhomogeneity are at the waveguide corners and near the boundary between the core and top cladding layer. However these two regions have opposite signs. Since overlap of mode with the top 
core/cladding interface, with negative strain gradient, is much larger than with the bottom corners of the sidewalls, which have positive gradient, to maximize the magnitude of FOM we seek to optimize overlap with negative strain gradient and minimizing overlap with positive regions. This can be achieved by complete etching of the ribs, illustrated in Figure 4.6 (f). In channel waveguides the bottom corners are further from the mode center, as compared to partially etched waveguides, and therefore their canceling effect is minimized.

The optimization procedure yields that the highest FOM can be achieved for the waveguide that is fully etched, $450 \mathrm{~nm}$ wide, 220 high and covered by $75 \mathrm{~nm}$ thick cladding layer. FOM results around structures with different waveguide width, cladding thickness and height are shown in Figure 4.7 (a), (b), and (c), respectively. Increasing waveguide width from $200 \mathrm{~nm}$ to about $450 \mathrm{~nm}$ leads to larger confinement factor and better overlap with strain gradient. However for widths larger than $450 \mathrm{~nm}$ there is less interaction of the mode with top corners. Thus, maximizing FOM implies a trade-off between the two main parameters, i.e., maximizing the strain gradient (smaller width) and the mode confinement (larger width) in the silicon core.

With an increasing cladding thickness, a progressively increasing force is applied to the waveguide core. However, the increase in the cladding thickness does not necessarily result in a higher strain gradient. An important contribution to the gradient is due to the corners of the cladding layer. The closer the cladding/air edge is to the core (i.e., the thinner the cladding is), the more prominent the stress influence becomes. Therefore, a trade-off between the maximal stress influence (thinner cladding) and maximal force 
(thicker cladding) yields an optimal cladding thickness. The optimum cladding thickness is found to be $75 \mathrm{~nm}$.

We investigate waveguides with various heights in Figure 4.7 (c) and find that indeed $220 \mathrm{~nm}$ SOI wafers have the optimum height for strain induced nonlinearity in silicon.

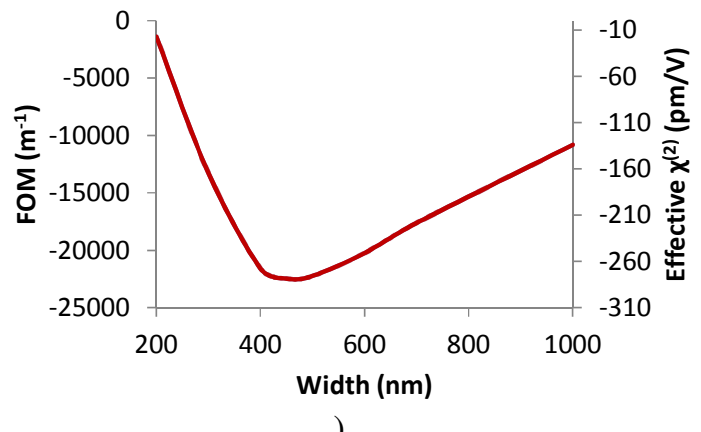

)

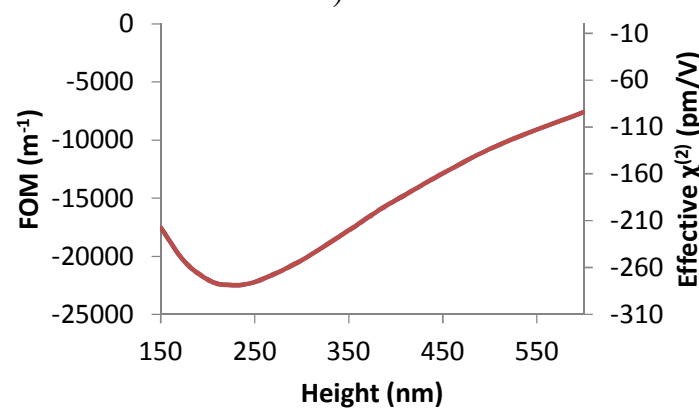

(c)

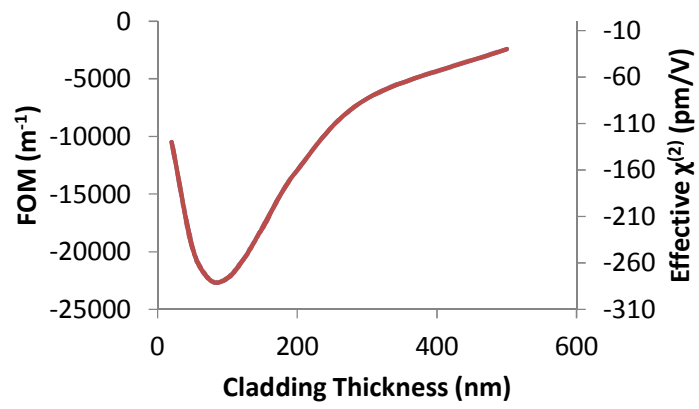

(b)

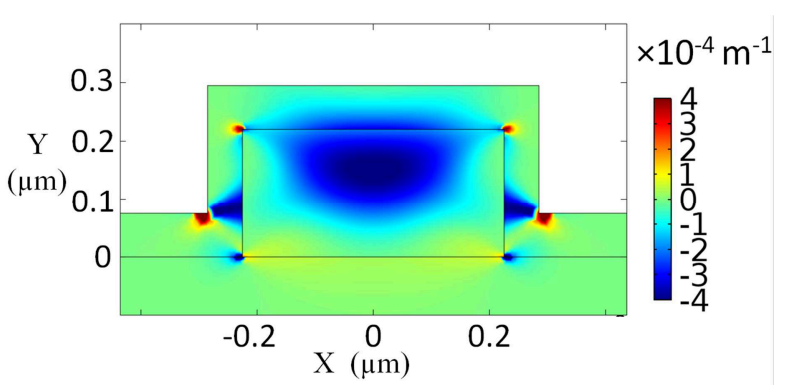

(d)

Figure 4.7 Optimization of strain induced effects in a nano-scale rib waveguide where cladding layer covers the waveguide sidewalls uniformly.

Figure of Merit (FOM) as function of (a) width, (b) cladding thickness, (c) height for a nano-scale rib waveguide with uniform cladding. The optimized parameters are $450 \mathrm{~nm}, 85 \mathrm{~nm}$ and $220 \mathrm{~nm}$ for width, cladding thickness and height, respectively. (d) Strain gradient and mode overlap for the optimized waveguide, achieving FOM $=22460 \mathrm{~m}^{-1}$ (or effective $\left.\chi^{(2)}=279 \mathrm{pm} / \mathrm{V}\right)$ ).

The corresponding FOM for the optimized device is $22,460 \mathrm{~m}^{-1}$ (or effective $\chi^{(2)}=323$ $\mathrm{pm} / \mathrm{V}$ ), which is a significant enhancement compared to FOM of $5810 \mathrm{~m}^{-1}$ (or effective $\chi^{(2)}=79.8 \mathrm{pm} / \mathrm{V}$ ) for the best structure reported in [13]. Relationship between FOM and $\chi^{(2)}$ is assumed to be linear, as the trend-line in Figure 4.3 suggests. 


\subsubsection{Optimization of strain induced effects in nano scale slanted rib waveguide}

In slanted rib waveguides, as illustrated in Figure 4.8 (a), the angle between the sidewall and the rib is an additional parameter that can used to obtain an optimized waveguide. Figure 4.8 (b) shows the magnitude of FOM decreases as side wall angle approaches $20^{\circ}$ from $90^{\circ}$. We can see in Figure 4.8 (c) that for smaller angles the positive gradients at the bottom are further away from the dominant negative gradient at the top and middle of the waveguide. However this does not result in improved FOM since as the bottom of the waveguide gets larger and larger the mode center moves down and away from the top interface of the waveguide and does not interact with the top interface in an optimum way. The net result is a decrease in magnitude FOM when $\theta<90^{\circ}$. Figure 4.8 (d) shows the mode overlap with the strain gradient for a waveguide with $\theta=48^{\circ}$. 


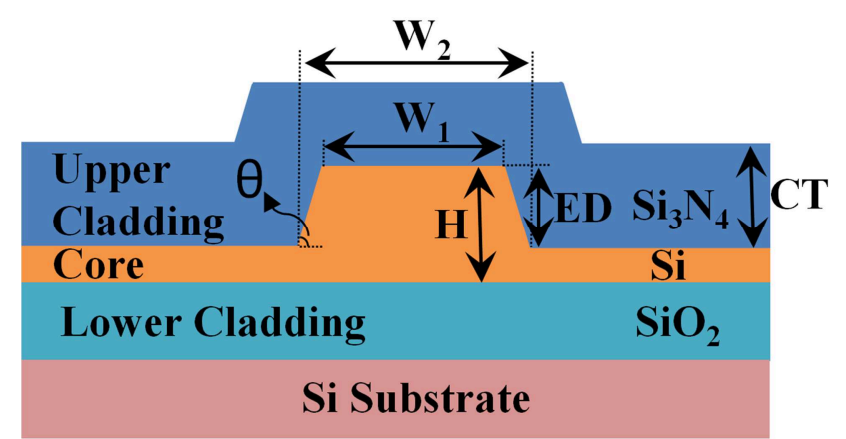

(a)

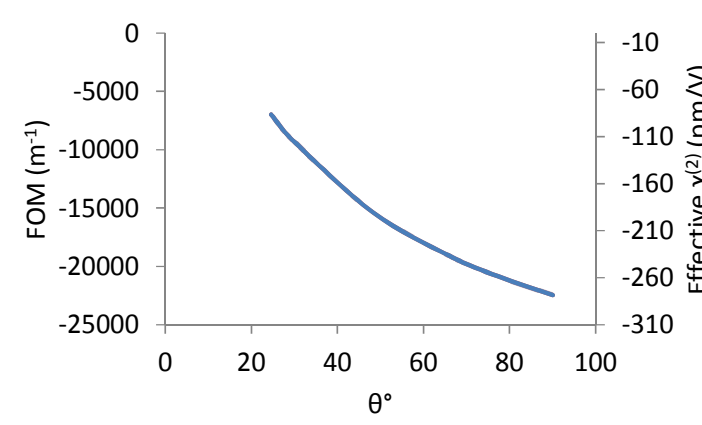

(b)

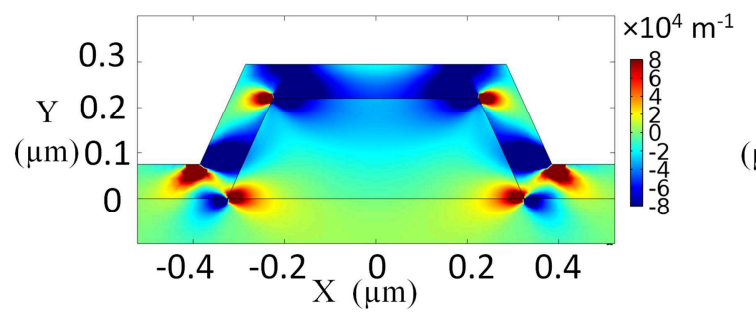

(d)

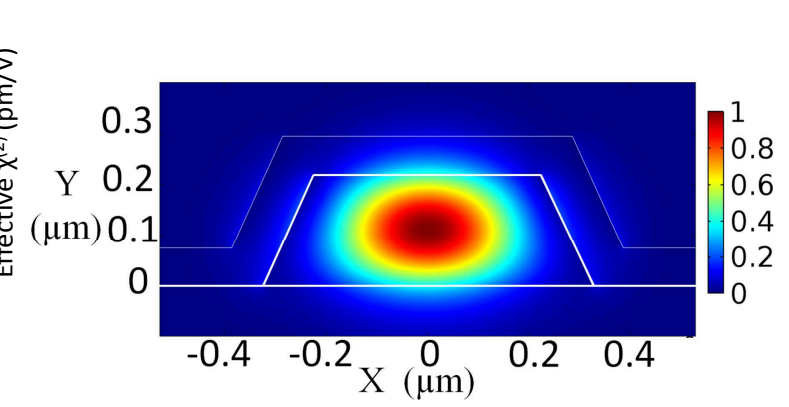

(c)

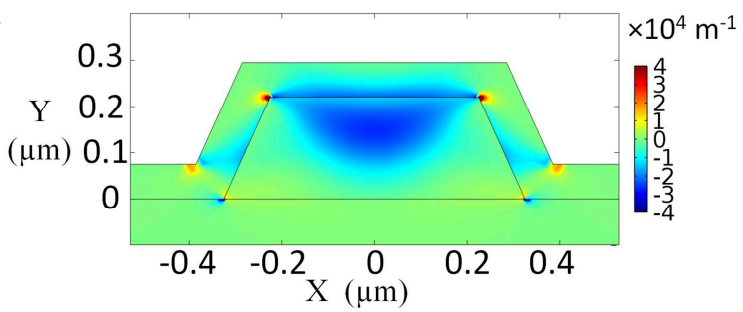

(e)

Figure 4.8 Stress induced effect for a $450 \mathrm{~nm}$ wide slanted channel waveguide.

(a) Geometrical parameters for a slanted rib waveguide. (b) FOM as a function of sidewall angle where $\mathrm{W}_{1}=450 \mathrm{~nm}, \mathrm{H}=\mathrm{ED}=220 \mathrm{~nm}, \mathrm{CT}=85 \mathrm{~nm}$ and $\mathrm{W}_{2}$ varies with $\theta$. (c), (d) and (e) show strain gradient distribution, fundamental mode distribution and overlap of strain gradient and the mode profile for waveguide with $\mathrm{W}_{1}=400$ and $\theta=48^{\circ}$.

\subsection{Material-induced stress}

Other than the geometrical parameters of the waveguide discussed above, the mechanical properties of the $\mathrm{Si}_{3} \mathrm{~N}_{4}$ layer also change the strain distribution in the waveguide and the induced $\chi_{\mathrm{Si}}{ }^{(2)}$. It is possible to change material properties of $\mathrm{Si}_{3} \mathrm{~N}_{4}$ by modifying deposition conditions [46]. In this section we investigate the effect of thermal expansion 
coefficient (TEC), intrinsic stress, and Young's modulus of $\mathrm{Si}_{3} \mathrm{~N}_{4}$ on FOM. Unless otherwise stated the optimized channel waveguide found in section 4.3.2, namely width = $450 \mathrm{~nm}$, cladding thickness $=75 \mathrm{~nm}$, and height $=220 \mathrm{~nm}$, is used in the following.

\subsubsection{Thermal Expansion Coefficient}

Thermal expansion coefficient of $\mathrm{Si}_{3} \mathrm{~N}_{4}\left(\alpha_{\mathrm{Si3N} 4}\right)$ can vary from $\sim 2$ to $\sim 4 \mu \mathrm{K}^{-1}$ depending on various deposition conditions, as discussed in Chapter 3. For silicon TEC is $\alpha_{\mathrm{Si}}=3.6$ $\mu \mathrm{K}^{-1}$. If $\alpha_{\mathrm{Si3N} 4}>\alpha_{\mathrm{Si}}$ the resulting thermal stress is tensile and if $\alpha_{\mathrm{Si} 3 \mathrm{~N} 4}<\alpha_{\mathrm{Si}}$ then the resulting thermal stress is compressive in the nitride layer. The thermal stress is added to intrinsic stress when calculating the total stress, as expressed in equation (1.3). Figure 4.9 (a) shows how thermal stress adds to or subtracts from the intrinsic stress of $+1 \mathrm{GPa}$ for various values of $\alpha_{\mathrm{Si3N} 4}$. The change in total stress results in a linear change in strain gradient and FOM, as seen in Figure 4.7 (b).

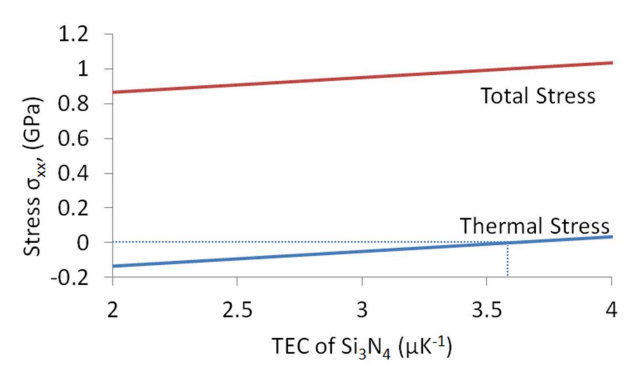

(a)

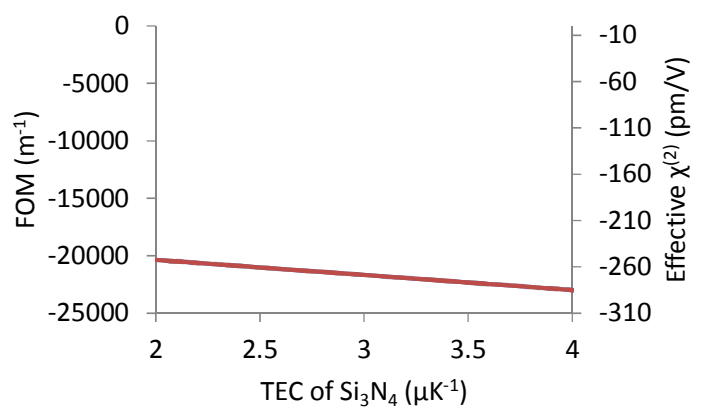

(b)

Figure 4.9 Linear relationship between thermal expansion coefficient (TEC) of $\mathrm{Si}_{3} \mathrm{~N}_{4}$ and (a) total stress, and (b) FOM.

When TEC of $\mathrm{Si}_{3} \mathrm{~N}_{4}$ is $3.6 \mu \mathrm{K}^{-1}$, thermal stress is nearly 0 .

\subsubsection{Intrinsic Stress}

The main source of stress in highly stressed $\mathrm{Si}_{3} \mathrm{~N}_{4}$ layers is the intrinsic stress. Therefore it is expected that any change in intrinsic stress strongly changes the strain distribution inside the silicon waveguide. Figure 4.10 shows the change in FOM as a function of 
intrinsic stress. The inverse symmetry around $\sigma_{\mathrm{xx}}=0 \mathrm{GPa}$ in the figure shows the sign change in the intrinsic stress merely changes the sign of FOM, and hence the induced effective $\chi^{(2)}$, and not its magnitude. This observation is different from what is claimed in [14], where the authors suggest that a sign difference between stressing layer and the BOX layer is required to obtain a high strain gradient. Our simulations show existence of a strong strain gradient inside the waveguide whenever a strong stress is applied from the overlaying layer, regardless of its sign.

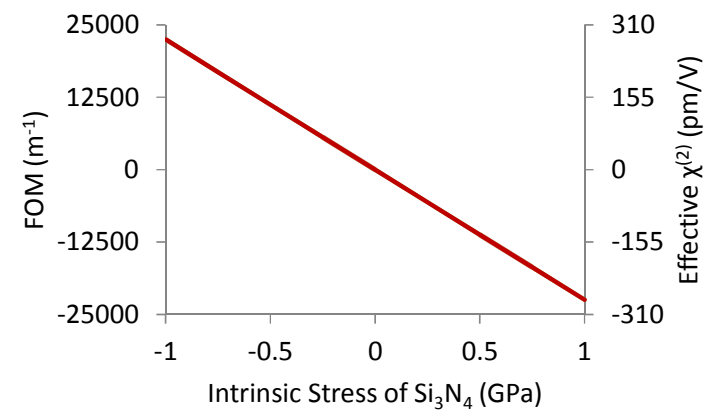

Figure 4.10 Linear relationship between intrinsic stress of $\mathrm{Si}_{3} \mathrm{~N}_{4}$ and FOM.

\subsubsection{Young's modulus}

A wide range of Young's modulus for silicon nitride $\left(E_{\mathrm{Si3N} 4}\right)$ has been reported in the literature [41], [46], [47], suggesting that the deposition conditions can be used to tune this parameter. Here we examine how change in $E_{\mathrm{Si} 3 \mathrm{~N} 4}$ affects FOM. As expressed in equations (3.2) and (3.3) Young's modulus affects the stress-strain relationship as well as the total stress in the material. Previously we showed TEC and total stress of $\mathrm{Si}_{3} \mathrm{~N}_{4}$ are both linearly related to FOM. We also observed, in Figure 4.9 (a), that choosing a thermal expansion coefficient similar to silicon, i.e. $\alpha_{\mathrm{Si3N} 4}=3.610^{-6}\left(\mathrm{~K}^{-1}\right)$, minimizes thermal stress in $\mathrm{Si}_{3} \mathrm{~N}_{4}$. In that case, the total stress is almost entirely due to intrinsic stress. This choice of material property helps to distinguish effect of Young's modulus on stressstrain relationship from its contribution to thermal stress. In other words we can now 
investigate how Young's modulus influences the FOM for constant total stress. Figure 4.11 (a) shows for $\alpha_{\mathrm{Si} 3 \mathrm{~N} 4}=3.6 \mu \mathrm{K}^{-1}$ although Young's modulus changes, there is negligible change in total stress, less than $10 \mathrm{MPa}$.

Figure 4.11 (b) illustrates the calculated FOM as function of Young's modulus of $\mathrm{Si}_{3} \mathrm{~N}_{4}$. Smaller Young's modulus leads to higher magnitude of FOM. This can be interpreted as follows: smaller Young's modulus in straining results in a softer cladding that facilitates the deformation of the silicon core layer. On the other hand high Young's modulus $\mathrm{Si}_{3} \mathrm{~N}_{4}$ results in a rigid cladding and resists deformation of the silicon core. In all previous sections when we calculated FOM for the experimentally demonstrated waveguides we assumed $E_{\mathrm{Si} 3 \mathrm{~N} 4}=63 \mathrm{GPa}$, which is in the lower range of values reported, resulting in high magnitude FOM according to Figure 4.11 (b). If the real value of $E_{\mathrm{Si} 3 \mathrm{~N} 4}$ is higher than 63 GPa, a substantial improvement in effective $\chi^{(2)}$ can be achieved by engineering the deposition of $\mathrm{Si}_{3} \mathrm{~N}_{4}$ to obtain a lower Young's modulus. This is indeed one of the most significant, and surprising, findings of this study.

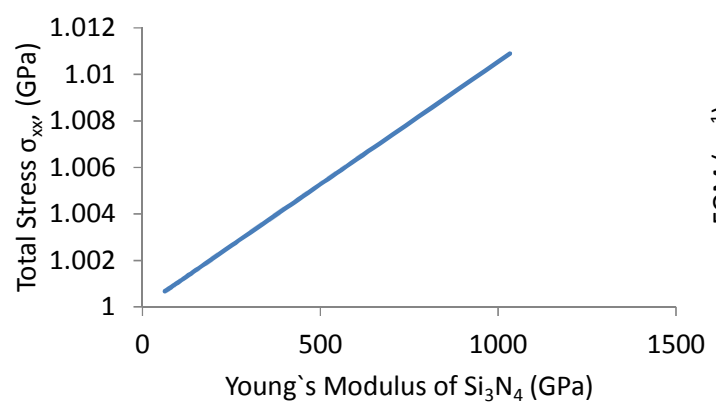

(a)

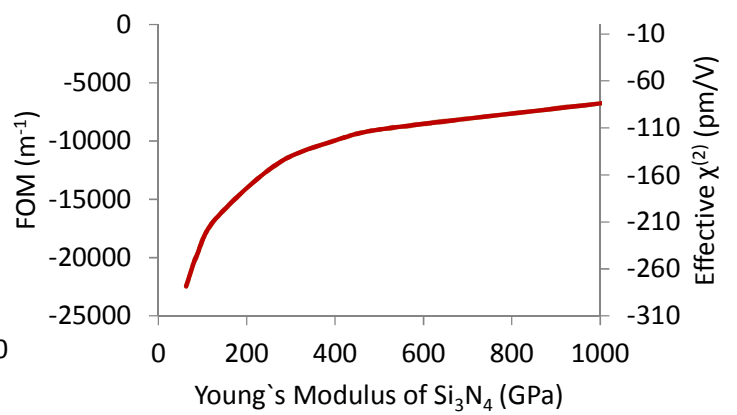

(b)

Figure 4.11 Effect of Young's modulus on (a) total stress and (b) FOM when thermal expansion coefficient (TEC) of silicon nitride is $3.6 \mu \mathrm{K}^{-1}$.

Note since TEC of straining layer is the same as silicon, thermal stress is minimal. The FOM values are for the optimized channel waveguide: width $=450 \mathrm{~nm}$, etch depth $=220 \mathrm{~nm}$, height $=220 \mathrm{~nm}$ and cladding

$$
\text { thickness }=85 \mathrm{~nm} \text {. }
$$




\subsubsection{Ideal material properties for cladding layer}

In conclusion, in addition to optimizing the geometrical parameters of the waveguide, mechanical properties of the silicon nitride stressor layer can be used to maximize strain inhomogeneity in silicon waveguides and FOM. We observe that for channel waveguides with uniform cladding, ideal material properties of silicon nitride stressing layer are: maximum total stress and minimum Young's modulus. It may be possible to obtain these attributes by modifying the deposition conditions.

\subsection{Effect of top $\mathrm{SiO}_{2}$ cladding on FOM}

In order to apply electric field to the waveguides electrodes are needed. The electrodes are typically made of titanium and gold and are deposited on a $\mathrm{SiO}_{2}$ layer. The $\mathrm{SiO}_{2}$ layer is deposited on top of the $\mathrm{Si}_{3} \mathrm{~N}_{4}$ straining layer to provide a platform and electrical isolation for the electrodes, as shown in Figure 4.12 (a). The thickness of $\mathrm{SiO}_{2}$ is several hundred nanometers. In this example we use $850 \mathrm{~nm}$ thick $\mathrm{SiO}_{2}$ similar to [13]. $\mathrm{SiO}_{2}$ is deposited using CVD and therefore its material properties vary depending on the deposition conditions, similar to $\mathrm{Si}_{3} \mathrm{~N}_{4}$. The properties of this layer are not reported from experiments. In previous sections we treated the problem without considering this top $\mathrm{SiO}_{2}$ layer, in order to reduce the number of unknown parameters. In this section we attempt to find an optimized geometry when a $\mathrm{SiO}_{2}$ layer is present.

Initially we have chosen all the material properties of the top $\mathrm{SiO}_{2}$ layer to be the same as $\mathrm{SiO}_{2}$ in the BOX layer, namely Young's modulus $E_{\mathrm{SiO} 2}=76.7 \mathrm{GPa}$, thermal expansion coefficient $\alpha_{\mathrm{SiO} 2}=0.54 \mu \mathrm{K}^{-1}$, Poisson's ratio $\nu_{\mathrm{SiO} 2}=0.186$, and no intrinsic stress which results in thermal (and total) stress of $\sigma_{\text {thermal }}=-305 \mathrm{MPa}$. 


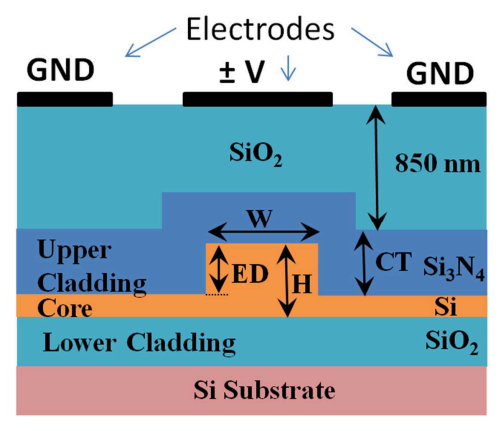

(a)

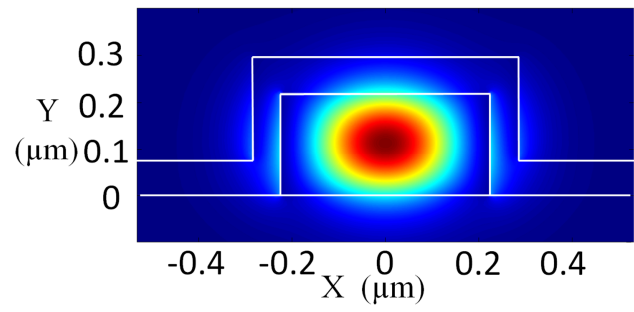

(c)

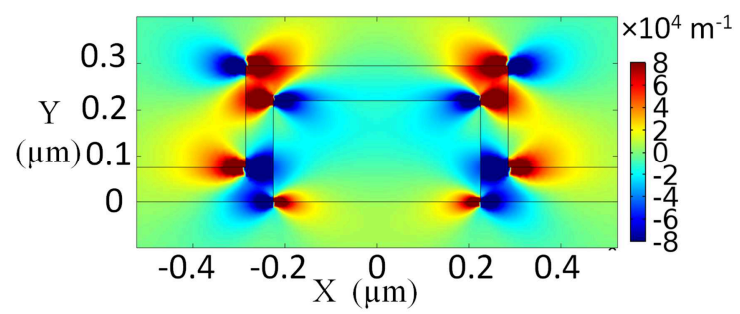

(b)

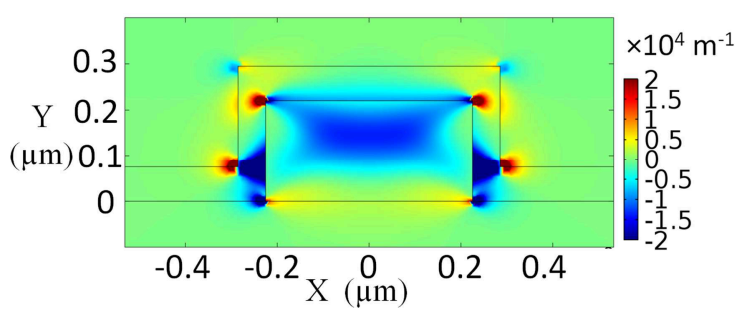

(d)

Figure 4.12 Strain induced effects in silicon rib waveguide with $\mathrm{Si}_{3} \mathrm{~N}_{4}$ straining layer and $850 \mathrm{~nm}$ $\mathrm{SiO}_{2}$ layer.

(a) Cross-section of a rib waveguide with modulating electrodes. (b) Strain gradient $\left(\partial \varepsilon_{x x} / \partial y\right)$. (c) Mode intensity profile. (d) Overlap of strain gradient and mode profile.

As can be seen in Figure 4.12 (b) the strain gradient profile features positive and negative regions which are similar to the case without top $\mathrm{SiO}_{2}$ layer. However in this case the positive gradients at the bottom corners are more pronounced relative to the negative regions. Since the overlap is overwhelmingly negative the overall FOM is much lower when top $\mathrm{SiO}_{2}$ layer exists.

Geometrical parametric scans around $\mathrm{W}=450 \mathrm{~nm}, \mathrm{ED}=220 \mathrm{~nm}$, and $\mathrm{CT}=85 \mathrm{~nm}$ were carried out. Figure 4.13 (a), (b) and (c), show FOM as a function of width, etch depth and cladding thickness, respectively. Plots include FOM values for waveguides with and without $\mathrm{SiO}_{2}$ layer. It can be seen that variation of $\mathrm{FOM}$ follows a similar pattern for both 
cases and the optimized geometrical parameters for both cases are approximately the same.

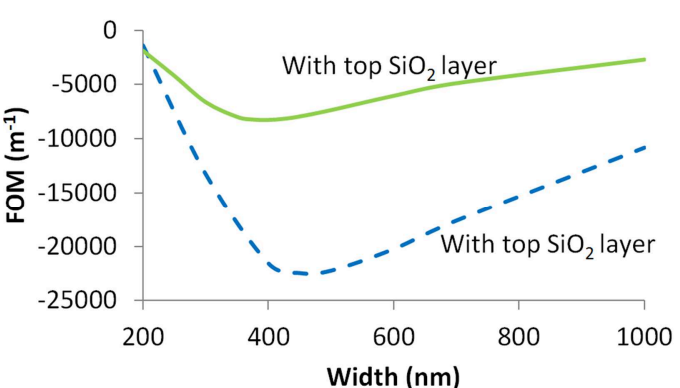

(a)

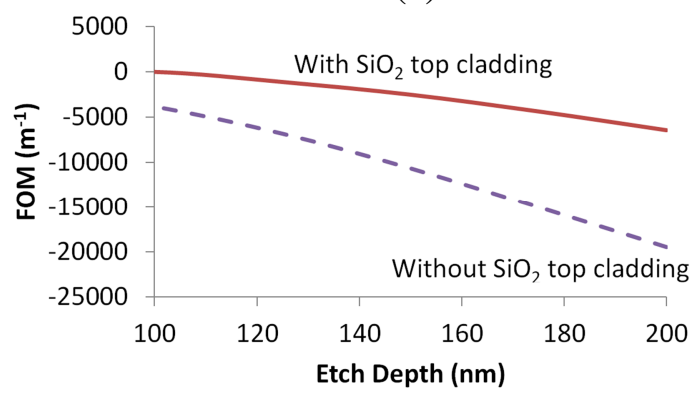

(c)

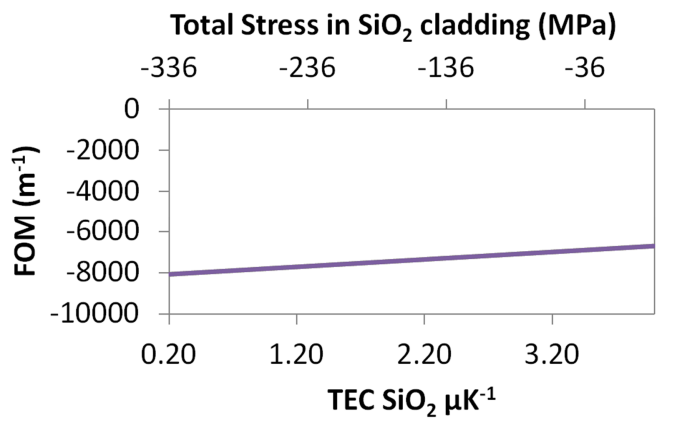

(e)

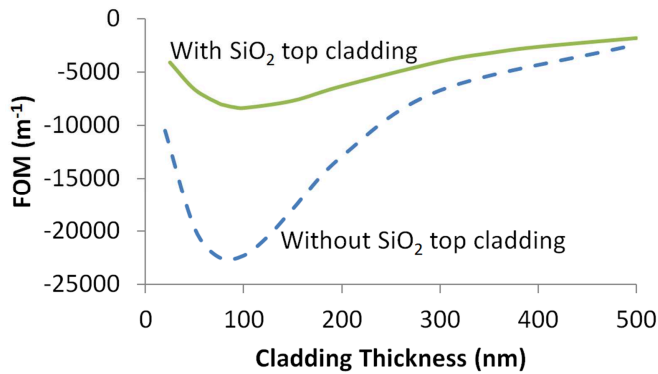

(b)

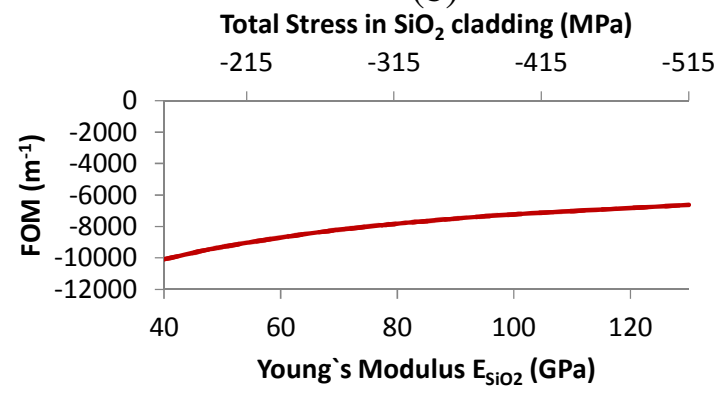

(d)

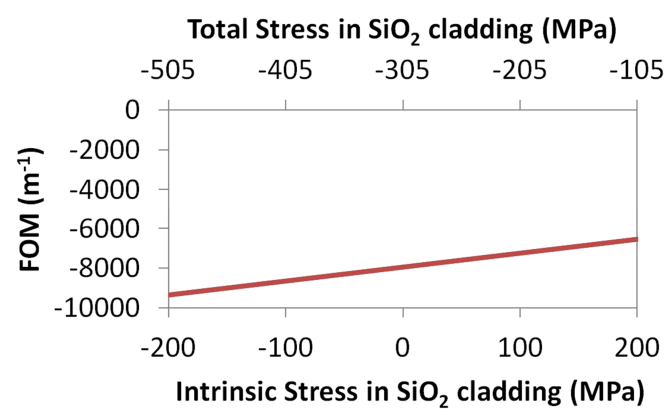

(f)

Figure 4.13 Optimization of strain induced effects in silicon rib waveguide with $\mathrm{Si}_{3} \mathrm{~N}_{4}$ straining layer and $850 \mathrm{~nm} \mathrm{SiO}_{2}$ layer.

FOM as a function of geometrical parameters:(a) Width (W), (b) Cladding Thickness (CT), (c) Etch Depth (ED) and material parameters for the top $\mathrm{SiO}_{2}$ layer: (d) Young`s modulus, (e) Thermal expansion coefficient, (f) Intrinsic stress. 
In addition, mechanical properties of the top $\mathrm{SiO}_{2}$ layer where altered to understand how they affect the FOM. Figure 4.13 (d), (e) and (f) show FOM as a function of Young`s modulus, thermal expansion coefficient and intrinsic stress of the top $\mathrm{SiO}_{2}$ layer. Similar to the conclusion reached for the $\mathrm{Si}_{3} \mathrm{~N}_{4}$ straining layer, here the highest (absolute) FOM is reached when Young`s modulus is small and (absolute) total stress is high.

\subsection{Position of the stress relief gap in straining layer}

Position of the gap in silicon nitride cladding, illustrated in Figure 4.2 (a), strongly influences the strain gradient distribution inside the silicon core. It is impossible to determine the exact location and angle of the gap from SEM images. Here we investigate how the change in location of the gap affects the strain gradient distribution and the FOM. Figure 4.14 (a) shows the geometrical parameters involved, including gap_x and gap_y which are relative $x$ and $y$ distance of the gap angle from the top corners of the waveguide, respectively. In Figure 4.14 (b) distribution of the strain gradient is shown when there is no gap in the cladding layer, regions of positive gradient and negative are about the same magnitude. By introduction of a gap that is deep enough to reach waveguide corners, i.e. gap_x $=$ gap_y $=0$, the magnitude of the positive gradient due to corners as well as the negative gradient at the top center of the waveguide become stronger, as illustrated in Figure 4.14 (c). Comparing FOMs for various widths in these configurations in Figure 4.14 (d) shows the existence of the gap increases the slope of the plot. This is because for large waveguides, say $700 \mathrm{~nm}$ wide, the mode predominantly overlaps with the top of the waveguide where gradient is strongly negative. As the waveguide gets smaller, strong positive gradients from the corners overlap more with the mode and eventually dominate the FOM. 


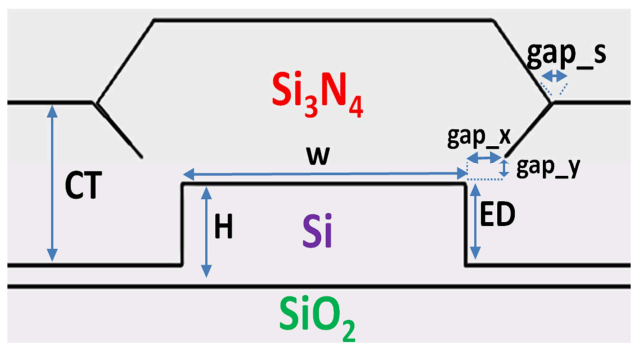

(a)

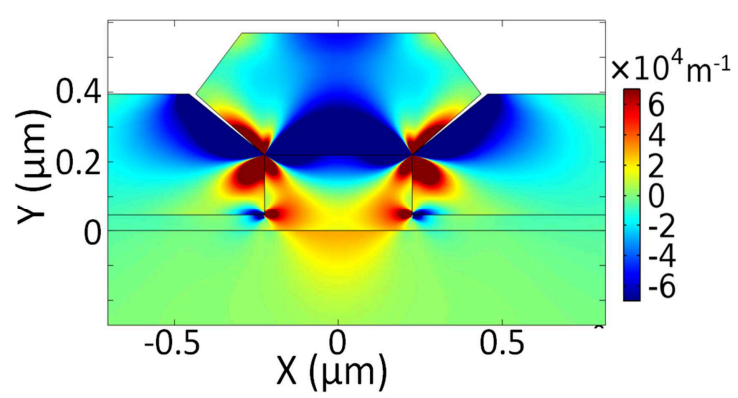

(c)

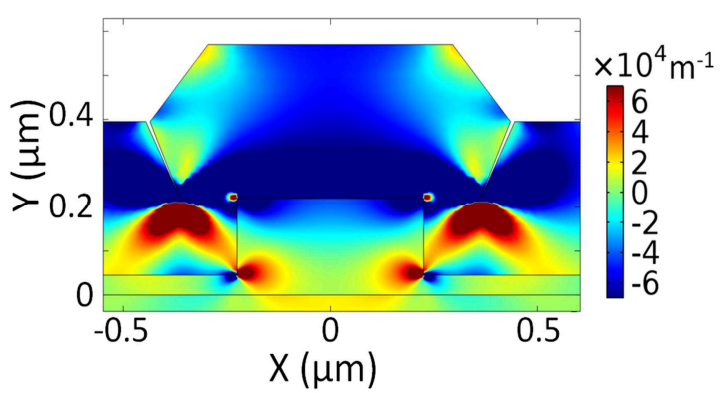

(e)

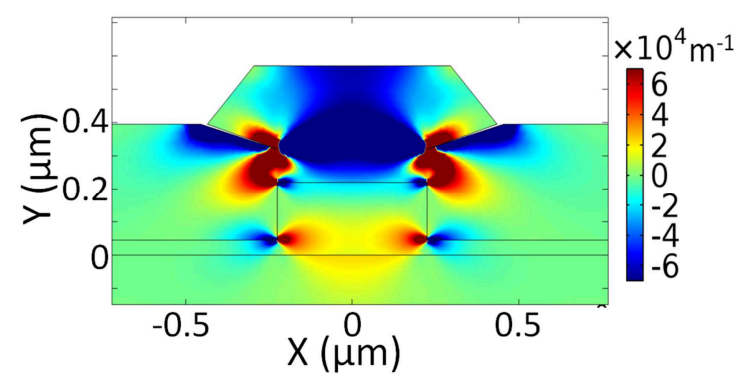

(g)

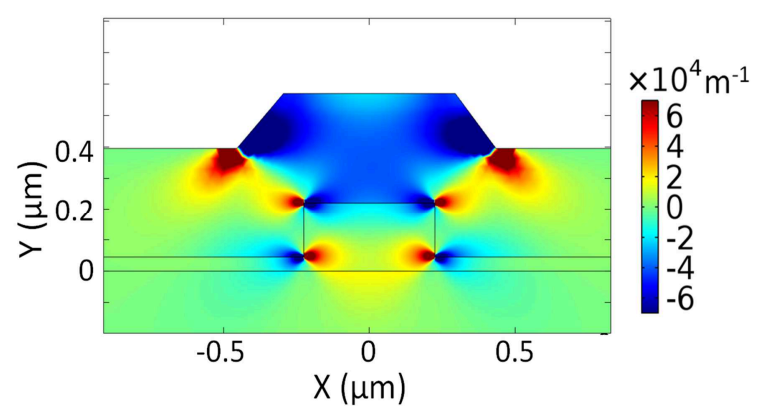

(b)

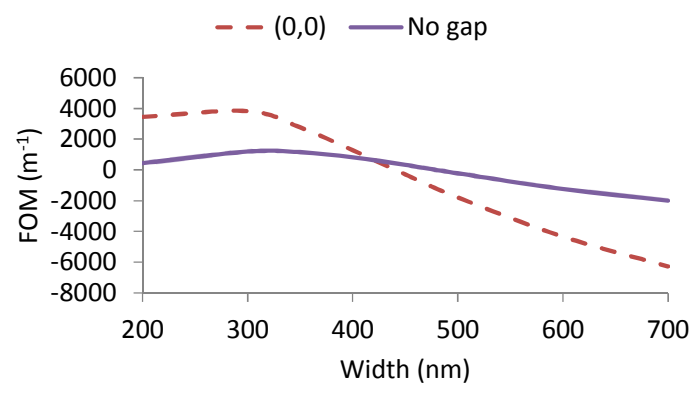

(d)

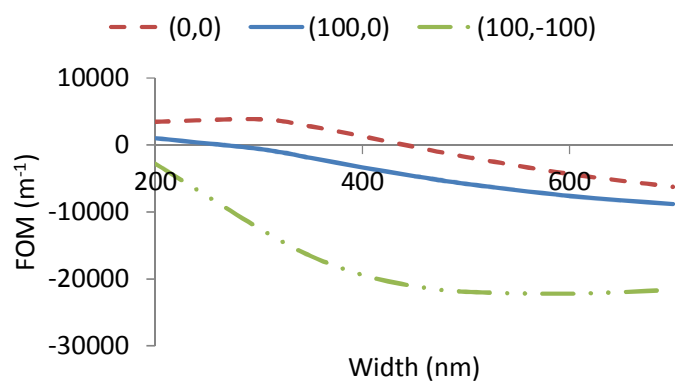

(f)

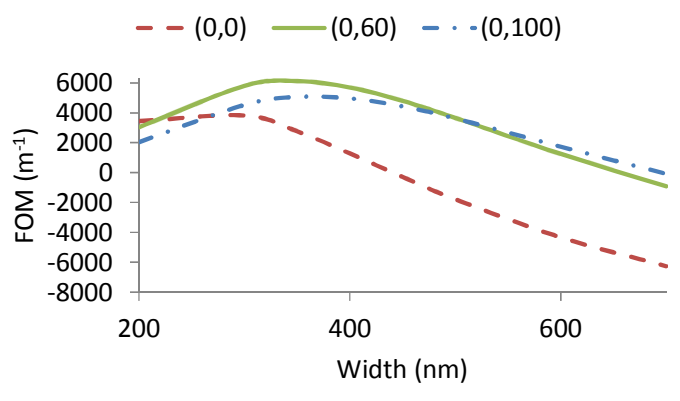

(h)

Figure 4.14 Influence of gap on distribution of strain gradient and FOM.

Numbers in brackets are (gap_x,gap_y) in nm. (a) Geometric parameters for the waveguide with gaps in the cladding. Strain gradient distribution for (b) no gaps in cladding, (c) gap ends are exactly at waveguide top corners, (e) gaps ends on the side of the waveguide wall, and (g) gaps ends on top of the waveguide.

(d), (f) and (h) show variation of FOM as a function waveguide width for different gap location. 
For the case when gap is located on the side of the waveguide walls and at the waveguide height the negative gradient at the top of the waveguide becomes more pronounced. This case is illustrated in Figure 4.14 (e) where gap_x $=100 \mathrm{~nm}$ and gap_y $=0$. If we move the gap corner to gap_x $=100 \mathrm{~nm}$ and gap_y $=-100 \mathrm{~nm}$, the negative gradient becomes even more effective, as seen in Figure 4.14 (f).

If the gap angle is located at the top of the waveguide, directly on top of the sidewalls, see Figure $4.14(\mathrm{~g})$ where gap_x $=0$ and gap_y $=100 \mathrm{~nm}$, then the negative gradient at the top of the waveguide becomes less pronounced. In this case the overall FOM remains positive for narrow waveguides up to about $600 \mathrm{~nm}$ wide, as seen in Figure 4.14 (h).

Overall we see that inclusion of the gap and its location has a very strong influence on strain gradient distribution and FOM. The existence of the gap is process dependent, and does not necessarily occur in all deposition conditions. To obtain reliable and predictable results it is advisable that the process is controlled in such manner to avoid the gap.

In section 4.3 we assumed the gap is located at the top of the waveguide walls, where gap_x=0 and gap_y=60 nm. This configuration was chosen since it provides the best correlation between FOM and experimentally observed effective $\chi^{(2)}$. 


\section{Chapter: Enhancement of Pockels Effect in Silicon Waveguides}

In this chapter we investigate alternative cladding materials to further enhance effective $\chi^{(2)}$. So far, all demonstrated strain induced silicon modulators use $\mathrm{Si}_{3} \mathrm{~N}_{4}$ or $\mathrm{SiO}_{2}$ as the stressor layer on the waveguide core [8], [9], [14], [15]. $\mathrm{Si}_{3} \mathrm{~N}_{4}$ is more commonly used because its stress parameters can be changed over a wide range by modifying the deposition conditions [46]. In most cases the stressor layer is deposited directly on the waveguide where it then also acts as the cladding layer. Both $\mathrm{Si}_{3} \mathrm{~N}_{4}$ and $\mathrm{SiO}_{2}$ have low refractive indices compared to silicon, $\mathrm{n}_{\mathrm{Si3N} 4}=1.98$ and $\mathrm{n}_{\mathrm{SiO} 2}=1.44$ while $\mathrm{n}_{\mathrm{Si}}=3.47$ at $\lambda=1550 \mathrm{~nm}$ [34]. A typical waveguide structure is shown in Figure 5.1 (a).The high refractive index contrast causes the mode to be tightly confined inside the silicon waveguide, as seen in Figure 5.1 (b), with the most intense part, residing at the center of the waveguide core. 


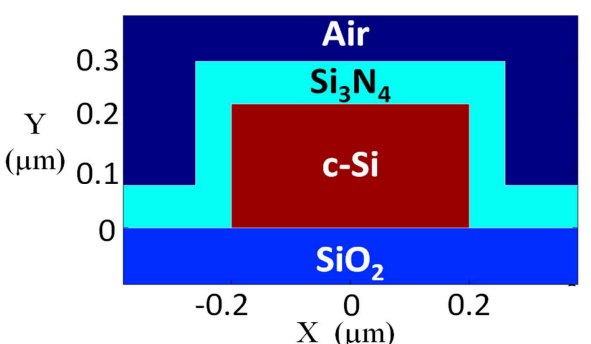

(a)

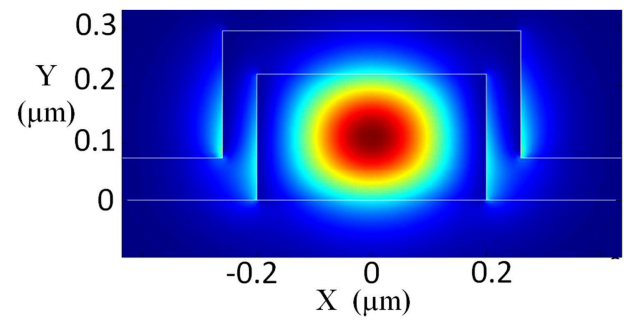

(b)

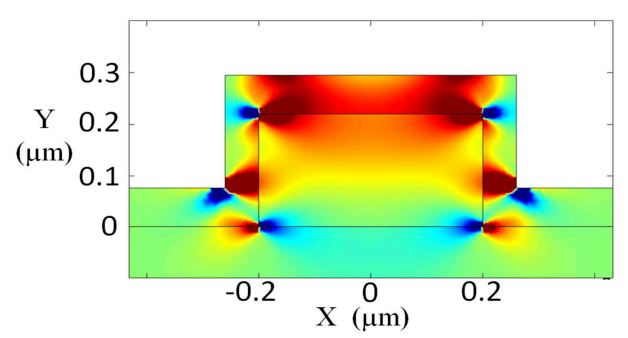

(c)

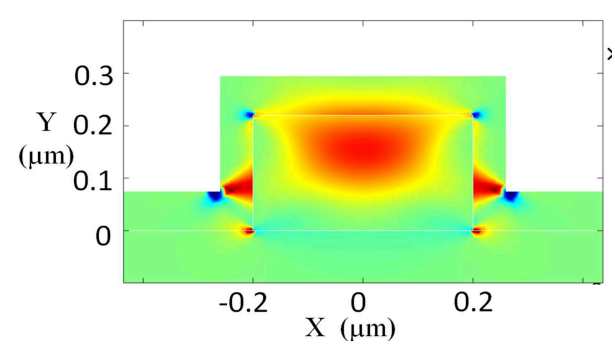

(d)

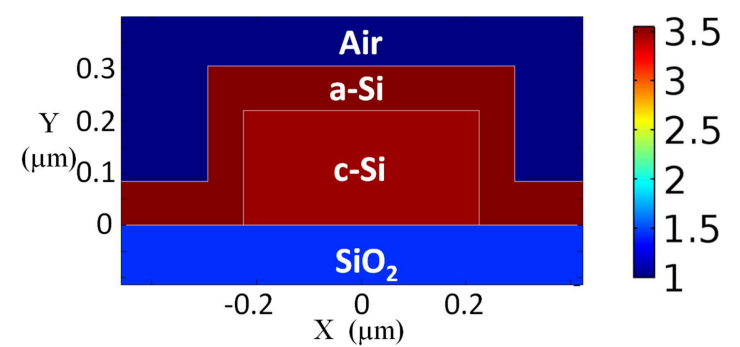

(e)

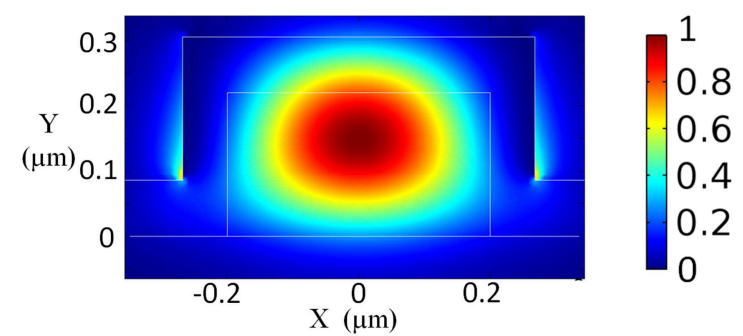

(f)

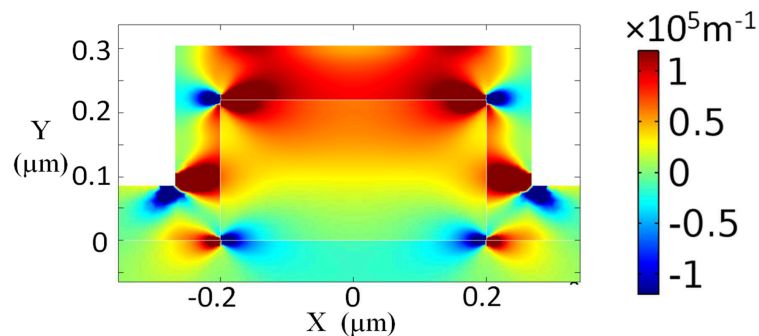

(g)

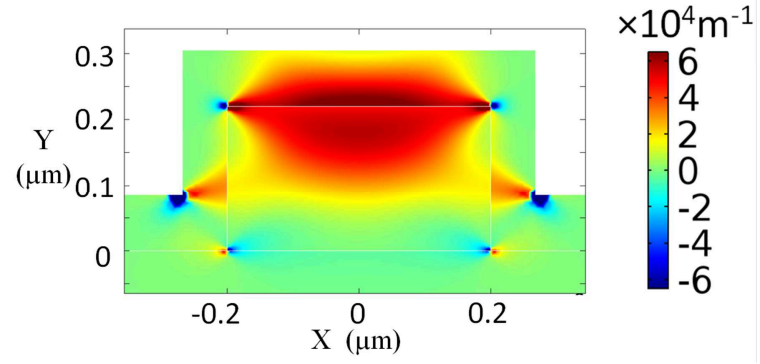

(h)

Figure 5.1 Comparison of strain-induced effects for waveguides with $\mathrm{Si}_{3} \mathrm{~N}_{4}$ and a-Si straining

layers.

Both waveguide are $400 \mathrm{~nm}$ wide on a $220 \mathrm{~nm} \mathrm{SOI}$, and feature straining layer thickness of $100 \mathrm{~nm}$.

For (a)-(d) the straining layer is $\mathrm{Si}_{3} \mathrm{~N}_{4}$ with +1 GPa stress. For (e)-(h) the straining layer is a-Si with -1

GPa stress. (a) and (e): Device structure and refractive index. (b) and (f): Intensity of waveguide normalized fundamental TE mode distribution. (c) and (g): Strain gradient distribution $\partial \varepsilon_{\mathrm{xx}} / \partial y$. (d) and (h): Overlap of strain gradient and intensity of normalized fundamental TE mode distribution. The overlap represents distribution of $\chi_{\mathrm{Si}}{ }^{(2)}$ inside the waveguide. Note in order to provide easier visual inspection (c) and (d) distributions are multiplied by -1 . Young's moduli are $\mathrm{E}_{\mathrm{Si3N} 4}=\mathrm{E}_{\mathrm{a}-\mathrm{Si}}=63 \mathrm{GPa} . \quad$ ) 
By finite element calculations of the induced strain distributions of the waveguide structure we have found that the magnitude of the strain gradient in silicon nanowires is strongest at the core/straining layer boundaries at the top and the corners of the sidewalls as demonstrated in Figure 5.1 (c) and discussed in previous chapter. The strain gradient in the center of the waveguide is much lower since the distribution decays rapidly as one moves away from its maxima at the sidewall corners and the top horizontal interface. Using low index straining layers, therefore, does not allow for the optimum interaction of the most intense part of the mode with regions of high strain gradient. Rather, optimizing the overlap of the mode with the waveguide edges can only be achieved by choosing waveguide geometry with sufficient mode delocalization. This in turn lowers the confinement factor, which limits the achievable effective $\chi^{(2)}$ of the waveguide. It is also undesirable from the point of view of waveguide scattering losses [48]. Figure 5.1 (d) shows the overlap of strain gradient and the optical mode which represents relative distribution of the $\chi_{\mathrm{Si}}^{(2)}$ inside the waveguide.

Since a high index straining layer effectively becomes part of the waveguide core, the waveguide structure can be designed such that the center of the optical mode is positioned in the area near the silicon/straining layer interface where the maximum strain gradients are located. There are several choices for straining layers that have comparable refractive index to silicon and are compatible with the CMOS technology, including silicon carbide ( $\mathrm{SiC}$ ), silicon germanium ( $\mathrm{SiGe}$ ) and amorphous silicon (a-Si) [49]-[51].

\subsection{Amorphous silicon straining layer}

In the following analysis, we use amorphous silicon as the straining material for demonstration of the concept, as it is a material that is commonly used in CMOS 
processing and does not require epitaxial growth. The structure schematic is shown in Figure $5.1(\mathrm{e})$.

Amorphous silicon has a range of Young's modulus ranging from 14 to $134 \mathrm{GPa}$ depending on deposition conditions and film thickness [23], [52]-[55]. In order to investigate purely optical effect of a-Si on FOM and be able to compare it with $\mathrm{Si}_{3} \mathrm{~N}_{4}$ we choose $E_{\mathrm{a}-\mathrm{Si}}=63 \mathrm{GPa}$, the same value used for $E_{\mathrm{Si} 3 \mathrm{~N} 4}$ in chapter 4 . Intrinsic stress in amorphous silicon stressing layers range from -1200 to $+350 \mathrm{MPa}$ [51], [56]. We choose -1 GPa intrinsic stress, so that results could be compared (in magnitude, not in sign) to $\mathrm{Si}_{3} \mathrm{~N}_{4}$ strained waveguides discussed in chapter 4 , where intrinsic stress was $+1 \mathrm{GPa}$.

Depending on the exact deposition technique and conditions, amorphous silicon has a refractive index of approximately $\mathrm{n}=3.55$. Since refractive index of a-Si is so close to crystalline silicon, light effectively "sees" a single composite waveguide core comprised of crystalline and amorphous silicon in which to propagate. In effect the boundary conditions that confine the mode are now at the strain layer-air interface as demonstrated in Figure 5.2. Therefore, by changing the thickness of the a-Si, we can engineer the mode distribution, as illustrated in Figure 5.2 (a) - (c). This provides for an additional degree of freedom in the waveguide design, which is not available for low index contrast straining layer designs where changing the straining layer thickness does not affect the mode distribution in a significant way. 


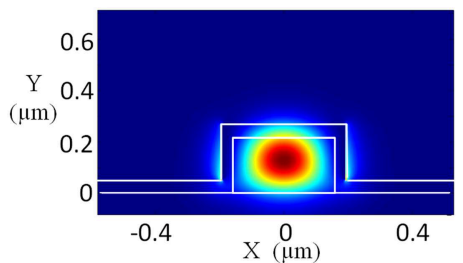

(a)

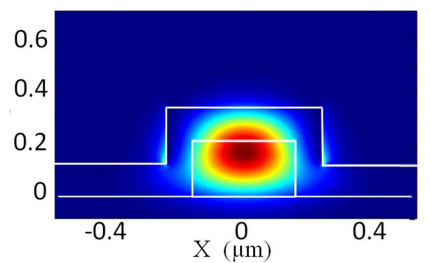

(b)

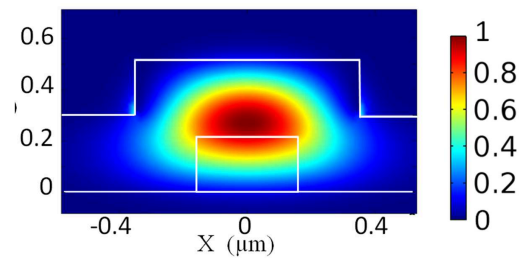

(c)

Figure 5.2 Normalized optical mode distribution in waveguides with a-Si straining layer. Straining layer thickness is 50, 125 and $300 \mathrm{~nm}$ for (a), (b) and (c), respectively.

Using the methodology outlined in chapter 4 we investigate various structures to enhance strain induced Pockels effect in a silicon waveguide with a-Si strain layer. For the commonly used $220 \mathrm{~nm}$ thick SOI waveguides, a multi-parameter optimization procedure yielded the following waveguide geometry to achieve the maximum value for the FOM: c-Si width $(\mathrm{c}-\mathrm{Si} \mathrm{W})=400 \mathrm{~nm}$, etch depth $(\mathrm{ED})=220 \mathrm{~nm}$ and straining layer thickness $(\mathrm{SLT})=$ $85 \mathrm{~nm}$. Figure 5.3 shows the dependence of the FOM for parameter scans around these optimal values for SLT (a), c-Si width (b) and etch depth (c). 


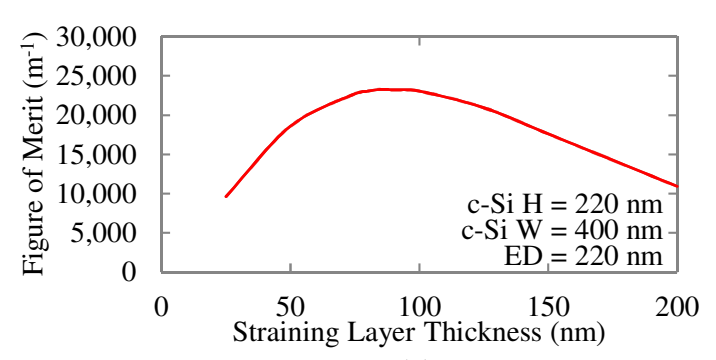

(a)

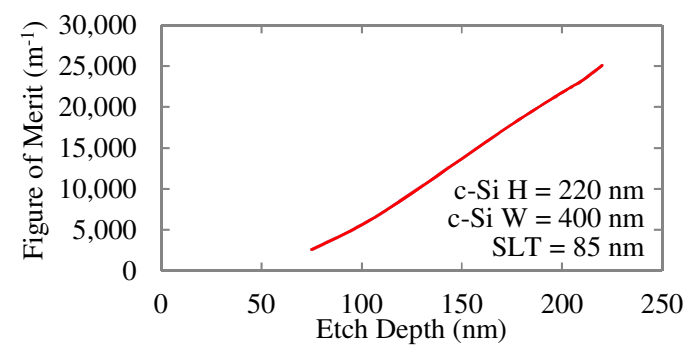

(c)

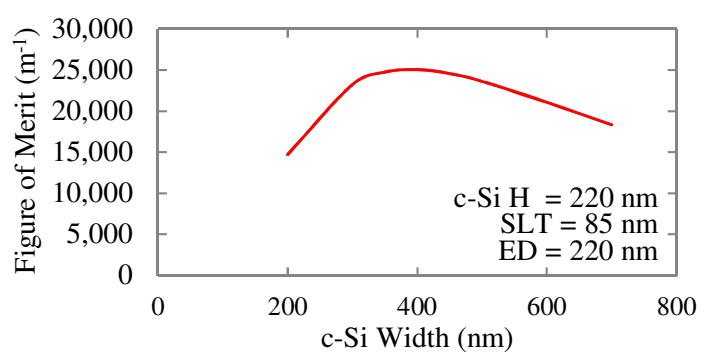

(b)

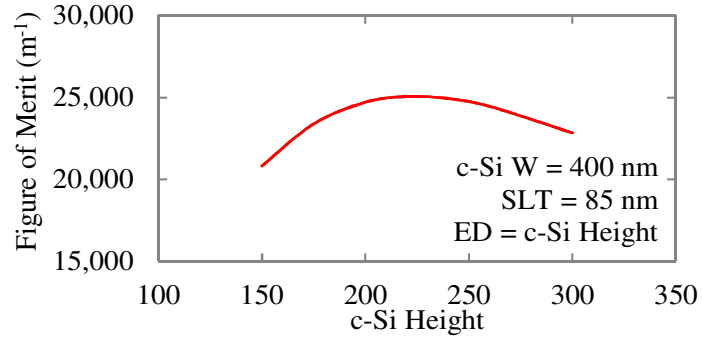

(d)

Figure 5.3 Optimization of strain induced effects for a nano scale channel waveguide with a-

Straining layer.

Figure of Merit (FOM) as a function of geometrical parameters for a silicon ridge nanowire with a-Si straining layer. (a) straining layer thickness (SLT). (b) c-Si width (c-Si-W). (c) etch depth (ED). (d) c-Si height (c-Si-H). The optimized parameters for straining layer thickness, c-Si width, etch depth, and c-Si height are $85 \mathrm{~nm}, 400 \mathrm{~nm}, 220 \mathrm{~nm}$ and $220 \mathrm{~nm}$ respectively.

FOM sharply increases when SLT increases from $10 \mathrm{~nm}$ up to about $85 \mathrm{~nm}$. There is progressively more overlap between the mode and the high strain gradient at the interface. For thicker a-Si the mode begins to be progressively more localized in the straining layer and therefore FOM decreases and eventually tapers off. As in the case of $\mathrm{Si}_{3} \mathrm{~N}_{4}$ devices discussed in chapter 4, in a-Si strained structures narrower waveguides have higher strain inhomogeneity. However the narrower waveguides lead to lower confinement in c-Si core. Therefore, there exists a trade-off between using narrower waveguides and maintaining mode localization inside the c-Si core. The optimum c-Si width is found to be $400 \mathrm{~nm}$. Although there is a strong strain gradient at the bottom corners of the sidewalls, the sign of gradient is opposite of the gradient at the interface. 
In a-Si strained waveguides overlap integral at the top interface dominates the FOM and etching lateral silicon completely results in a higher FOM, as seen in Figure 5.3 (c). We also explored waveguides with various heights, shown in Figure 5.3 (d). It is observed the optimal c-Si height (c-Si H) is indeed $220 \mathrm{~nm}$.

The highest FOM achieved for a channel a-Si strained waveguide is $\sim 25000 \mathrm{~m}^{-1}$, which is about $10 \%$ improvement over $\mathrm{Si}_{3} \mathrm{~N}_{4}$ strained waveguides studied earlier. This improvement is entirely due to difference of refractive index of a-Si and $\mathrm{Si}_{3} \mathrm{~N}_{4}$. It is possible to further improve FOM in a-Si straining layer waveguides by utilizing a lower Young's modulus. For instance in Figure 5.3 it is observed if we use $E_{\mathrm{a}-\mathrm{Si}}=40 \mathrm{GPa}, \mathrm{FOM}$ increases to $30100 \mathrm{~m}^{-1}$. This value is substantially higher than $5810 \mathrm{~m}^{-1}$ obtained for ridge waveguide reported in [13]. Assuming the material properties geometrical parameters used for calculating FOM in chapter 4 are close to the real experimental values, and relationship between FOM and $\chi^{(2)}$ stays linear beyond values in Figure 4.3, we can use equation (4.2) to obtain effective $\chi^{(2)}=373 \mathrm{pm} / \mathrm{V}$ which is higher than $\chi_{\mathrm{LiNbO} 3}{ }^{(2)}=360 \mathrm{pm} / \mathrm{V}$.

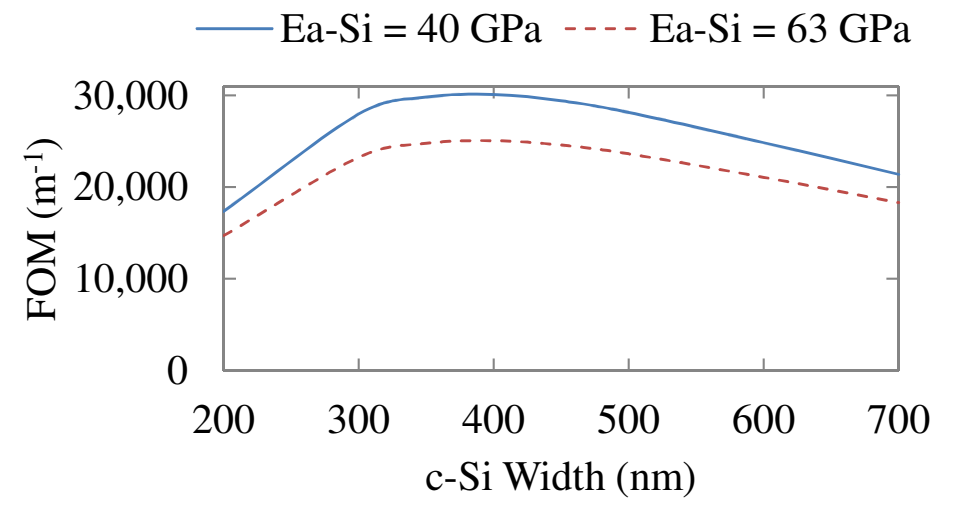

Figure 5.4 Figure of Merit as a function of width of the silicon core for $E_{\mathrm{a}-\mathrm{Si}}=40$ and $63 \mathrm{GPa}$. 


\subsection{Silicon germanium straining layer}

In the case of a-Si straining layer, discussed in previous section, only the part of the mode overlapping with c-Si contributes to the effective $\chi^{(2)}$ of the waveguide since amorphous materials do not exhibit significant Pockels effect.

Epitaxial techniques arrange atoms in single-crystal fashion upon a seed crystalline substrate, silicon for example. The lattice of the newly grown film duplicates that of the substrate [57]. It is expected an epitaxially grown crystalline overlayer, such as SiGe, the straining layer itself also exhibits a strain induced Pockels effect. This could potentially lead to a further enhancement of the overall effective $\chi^{(2)}$. In this section we investigate the potential to further enhance effective $\chi^{(2)}$ by epitaxially grown SiGe as a straining and complementary active layer.

Stress in SiGe layer grown on silicon is due to the $\sim 4 \%$ lattice mismatch between silicon and germanium crystals and can be several GPa in magnitude [49], [58]. Intrinsic strain of $\mathrm{Si}_{\mathrm{p}} \mathrm{Ge}_{1-\mathrm{p}}$, with silicon content $p$ ranging from $0 \%$ to $100 \%$, can be calculated using Vegard's law [49]:

$$
\varepsilon_{0}=\frac{(1-p) \mathrm{a}_{\mathrm{Ge}}+p \mathrm{a}_{S i}}{\mathrm{a}_{S i}}-1
$$

where $\mathrm{a}_{\mathrm{Ge}}$ and $\mathrm{a}_{\mathrm{Si}}$ are lattice constants of $\mathrm{Ge}$ and $\mathrm{Si}$, receptively. This holds true when there are no dislocations in the newly formed lattice. Total stress as a function of $\mathrm{Ge}$ content for a dislocation-free SiGe film is reported in Figure 5.5 (a).

An important limitation for SiGe stress layers is Ge content. Bandgap of Si and Ge are $1.1 \mathrm{eV}$ and $0.67 \mathrm{eV}$ at room temperature [59]. Bandgap of $\mathrm{SiGe}$ as a function of $\mathrm{Ge}$ content is illustrated in Figure 5.5 (b). At telecom wavelength of $\lambda=1550 \mathrm{~nm}$ photons have energy $0.8 \mathrm{eV}$ energy, according to $\mathrm{E}=h c / \lambda$, where $h$ is Plank's constant and $c$ is 
speed of light in vacuum. Figure 5.5 (b) shows that content of $\mathrm{Ge}$ in $\mathrm{SiGe}$ must be below $\sim 40 \%$ to avoid absorption of $1550 \mathrm{~nm}$ photons by the bandgap.
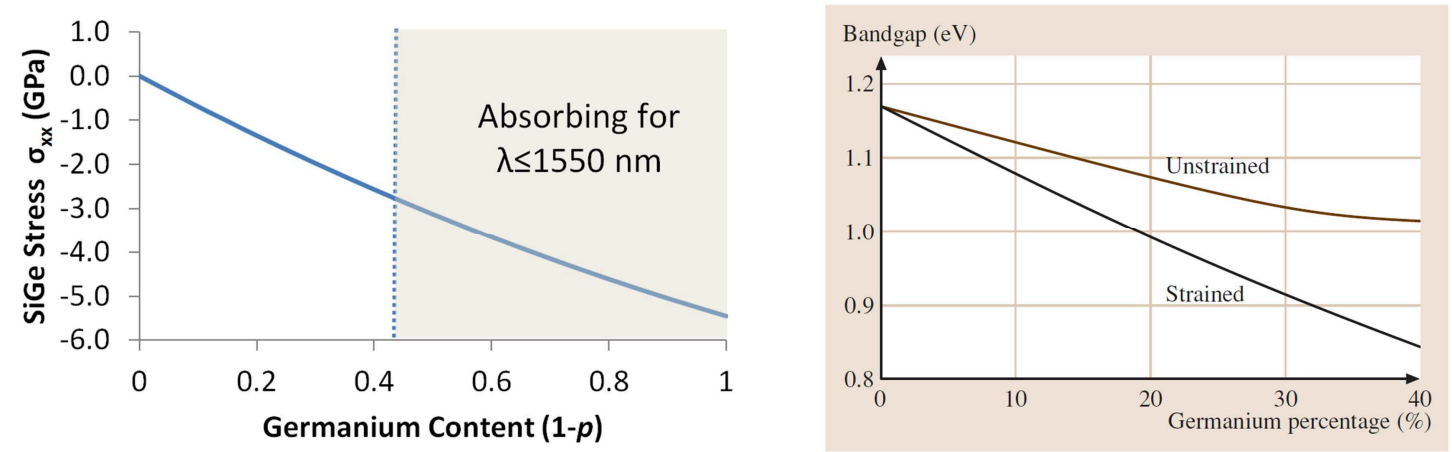

Figure 5.5 (a) Total stress in SiGe as a function of Ge content. (b) Bandgap energy of SiGe at 300 $K$ as a function of Ge content [59].

Highlighted section in (a) shows Ge content that leads to absorption for photons with $\lambda \leq 1550 \mathrm{~nm}$.

In order to make a conservative estimate and be able to compare the result of this section with the previous sections initially we assume film stress $\sigma_{\text {film }}=-1 \mathrm{GPa}$, corresponding to $p=0.85$.

The FOM needs to be modified to take into account the contribution of the SiGe layer. The new FOM is defined as:

$$
F O M=\frac{\iint_{S i}\left(\frac{\partial \varepsilon_{x x}}{\partial y}\right)|E|^{2} d x d y+s \iint_{S i G e}\left(\frac{\partial \varepsilon_{x x}}{\partial y}\right)|E|^{2} d x d y}{\int_{-\infty}^{\infty} \int_{-\infty}^{\infty}|E|^{2} d x d y}
$$

where $s$ is an unknown scaling factor that takes into account the different material response of SiGe layer depending on its content $p$. In general, mechanical and optical properties of SiGe layer vary linearly as functions of silicon and germanium content in 
the cladding, for example $E_{\mathrm{SiGe}}=p E_{\mathrm{si}}+(1-p) E_{\mathrm{Ge}}=126 \mathrm{GPa}$ for $p=0.85$. Here we use $p=$ 0.85 and assume $\mathrm{s}=\mathrm{p}$. Refractive index and extinction coefficient of germanium at 1550 are $\mathrm{n}_{\mathrm{Ge}}=4.275$ and $\mathrm{k}_{\mathrm{Ge}}=5.67 \times 10^{-3}[33]$.

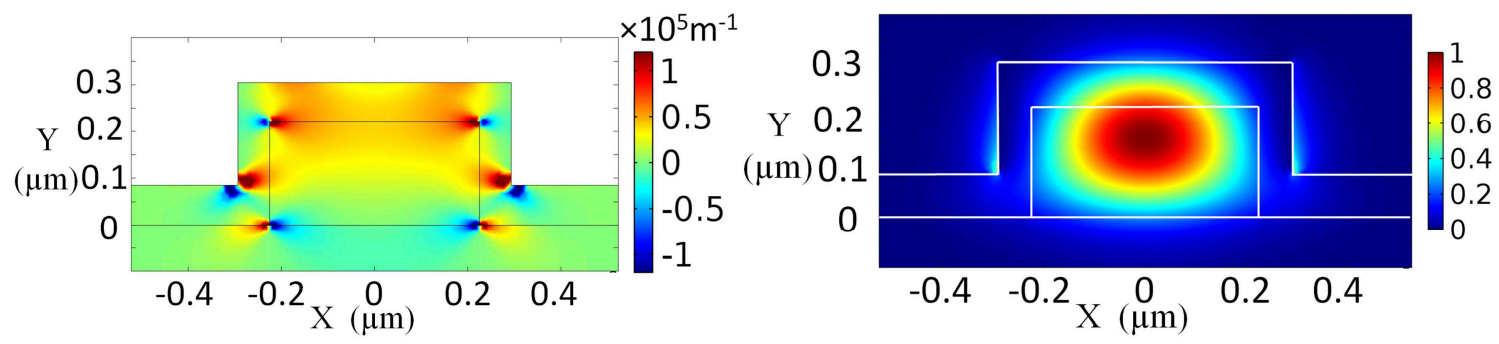

(a)

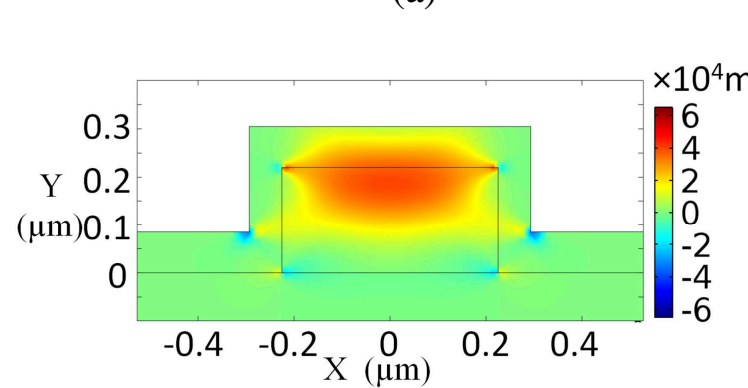

(c)

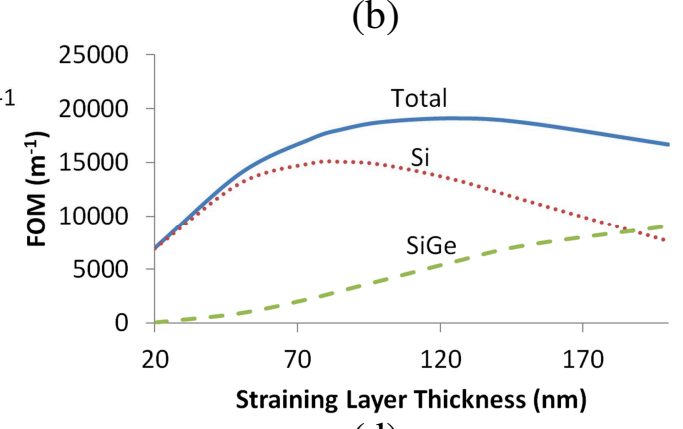

(d)

Figure 5.6 Strain induced effects for silicon waveguide with SiGe straining layer.

(a) Strain gradient $\partial \varepsilon_{x x} / \partial y$. (b) Mode intensity profile. (c) Strain gradient and mode intensity overlap. FOM as a function of SiGe straining layer thickness. Dashed and dotted lines show contributions of $\mathrm{SiGe}$ and $\mathrm{Si}$ layers to FOM, respectively. Solid line is the total FOM.

Interestingly, although there is a contribution from the SiGe layer, the total FOM is not more than what was obtained for a-Si devices. This is due to higher Young`s modulus of SiGe epi layer, compared to $E_{\mathrm{a}-\mathrm{Si}}=63$ or $40 \mathrm{GPa}$, that decreases the FOM as was discussed in section 4.4.3.

By increasing the intrinsic stress of SiGe to $-2 \mathrm{GPa}$, corresponding to $p=0.7$, a significant increase is achieved in FOM=35600 $\mathrm{m}^{-1}$, as illustrated in Figure 5.7. 


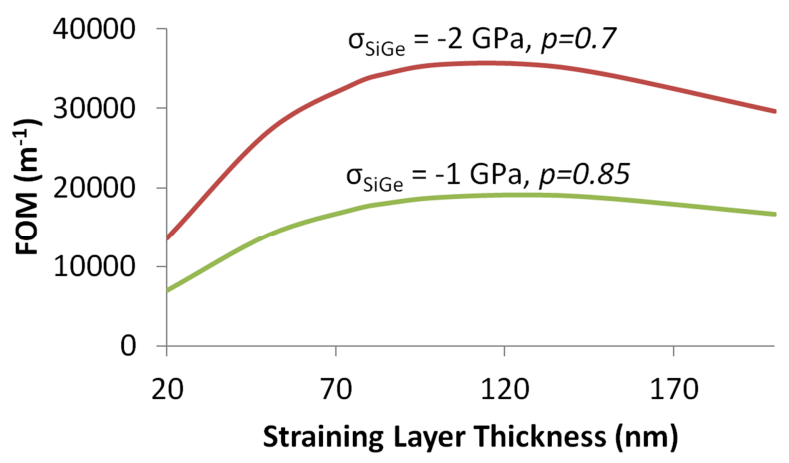

(a)

Figure 5.7 FOM as a function of straining layer thickness for filmstress of $\sigma_{\text {film }}=-1$ GPa and -2 GPa, corresponding to $p=0.85$ and 0.7 , respectively.

We also explored the slot $\mathrm{SiGe}$ waveguides as an alternative to the regular channel waveguides. The corners are the main sources of strain inhomogeneity in the waveguide; therefore introducing a slot is expected to increases the overall FOM. The simulation results for a slot waveguide with -1 GPa stressing layer $(p=0.85)$ are shown in Figure 5.8 (a) to (d). From slot depth of $75 \mathrm{~nm}$ to $125 \mathrm{~nm}$, there is a marginal improvement of FOM caused by the extra gradient resulting from the slot. However the slot introduces both positive and negative strain gradients and the overall integral decreases for slots deeper than $125 \mathrm{~nm}$. 


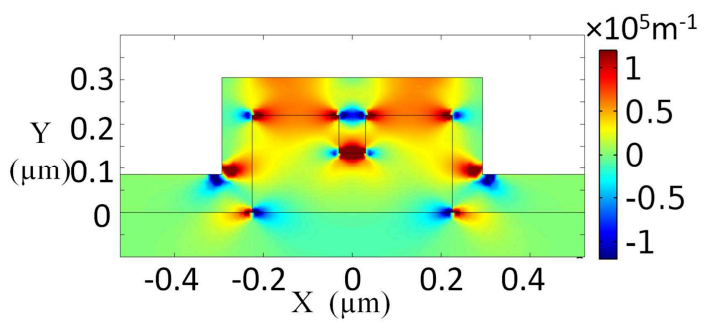

(a)

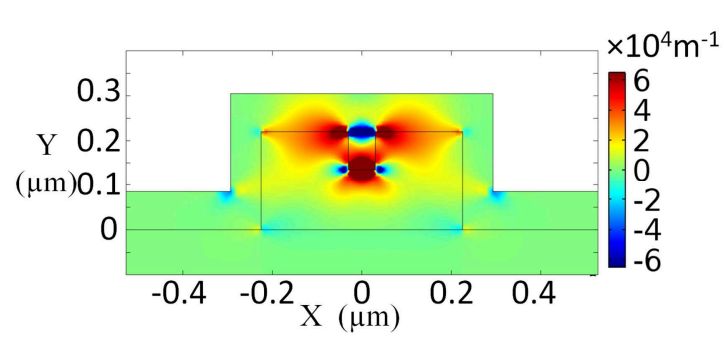

(c)

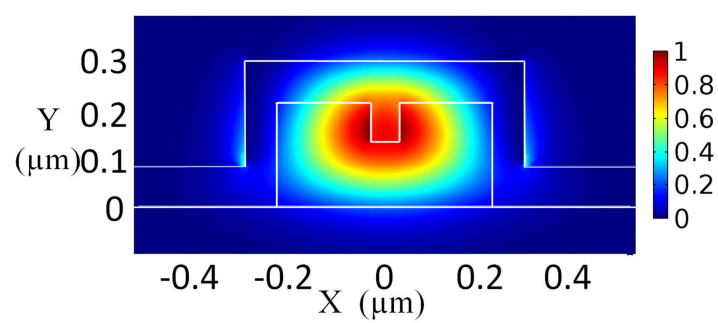

(b)

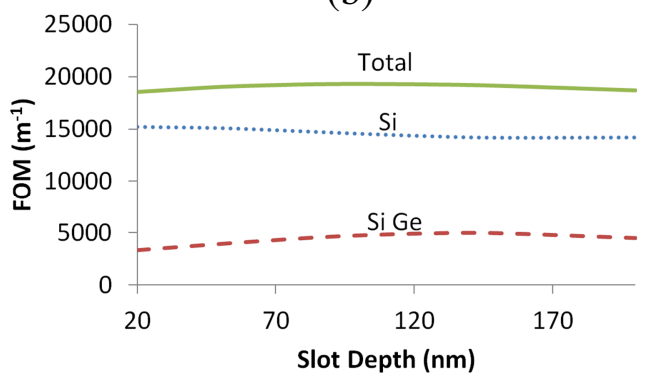

(d)

Figure 5.8 Strain induced effects for a nano scale slot channel waveguide.

(a) Strain gradient $\partial \varepsilon_{x x} / \partial y$. (b) Mode intensity profile. (c) Strain gradient and mode intensity overlap.

(d) FOM as a function of SiGe straining layer thickness. Dashed and dotted lines show contributions of SiGe and Si layers, respectively. Solid line is the total FOM. 


\section{Chapter: Conclusion and Future Work}

\subsection{Summary}

In this thesis a detailed investigation of strain induced Pockels effect in silicon waveguides was carried out. A new Figure of Merit (FOM) was proposed that allows one to characterize the strength of the Pockels effect in silicon waveguides structures numerically based on overlap of strain gradient and optical mode intensity utilizing relatively simple calculations based on the Finite Element Method. The FOM is a design tool that circumvents the problem of discrepancy between theoretically calculated and experimentally observed second order nonlinearity $\chi^{(2)}$ in silicon waveguides. We illustrated the proposed FOM has an excellent correlation with experimentally measured effective $\chi^{(2)}$ values reported for various structures currently reported in the literature. Using this approach, we studied the influence of the waveguide geometry on the FOM for silicon nanowire ribs as reported in the literature. By optimizing the waveguide crosssection, we showed a near 4 fold enhancement in the FOM is possible compared to the most efficient devices reported to date with associated FOM of $5810 \mathrm{~m}^{-1}$. By geometrical optimization an FOM of $22460 \mathrm{~m}^{-1}$ or effective $\chi^{(2)}$ as high as $279 \mathrm{pm} / \mathrm{V}$ was obtained. The accuracy of the numerical predication is strongly influenced by the induced strain distribution in the silicon waveguide from the cladding layer (i.e., silicon nitride). The exact strain distribution profiles are determined by specific mechanical and geometrical parameters such as the precise location of the gaps in the silicon nitride cladding layer. However, some of these parameters are not well documented in the experimental data sets used for the verification and validation of our numerical model. 
In addition to geometrical parameters of the waveguide, effects of mechanical and optical properties of straining layer on the induced Pockels effect were also investigated. We conclude that in order to obtain the highest effective $\chi^{(2)}$ in silicon the ideal straining layer has a high level of total stress either compressive or tensile, $1 \mathrm{GPa}$ in magnitude or more, a low Young's modulus, $<100 \mathrm{GPa}$, and a refractive index similar to silicon. The latter offers an additional degree of freedom in optimizing the mode overlap integral by engineering the confinement of the optical mode in the core. We showed that an amorphous silicon strained silicon waveguide can achieve FOM of $30100 \mathrm{~m}^{-1}$ or effective $\chi^{(2)}=373 \mathrm{pm} / \mathrm{V}$. Dislocation free silicon germanium cladding with a high stress level of 2 GPa in magnitude can also be used to further increase FOM to $\sim 35,600 \mathrm{~m}^{-1}$.

A key finding of this study is that the sign of strain gradient distribution inside the silicon waveguide changes vertically across the waveguide. Especially for the partially etched waveguides where the bottom corners of the vertical walls overlap substantially with the mode, the sign difference between the top interface and bottom corners limits the achievable $\chi^{(2)}$. This point has only been mentioned marginally in the current literature [11] and is assumed to be a negligible factor. However our investigation shows that it is a significant aspect of designing an optimized strain induced Pockels effect in silicon waveguides. We discovered a much higher overlap integral is achievable if the waveguide is fully etched and the bottom corners do not overlap substantially with the mode. With the fully etched waveguide design the main source of strain gradient (and Pockels effect) is the at the top core/cladding interface. 


\subsection{Future work}

Indeed, the comparison between our simulations and experimental results was limited to a few studies currently available in the literature. Efforts were made to conduct in-house experiments to collect additional data at the National Research Council, however due to the availability of the deposition tools and the limited resources accessible for the fabrication, samples were not ready at the time of this thesis defense. An example of the designed mask can be found in Appendix B . In the future, when more experimental data is available, the correlation plot presented in Figure 4.3 could be revisited and its accuracy examined and improved.

It is plausible that the Pockels effect can also be induced in silicon waveguides by removal of the symmetry in the horizontal direction. In horizontally symmetrical waveguides studied in the current work, $\partial \varepsilon_{y y} / \partial x$ vanishes. If the straining layer is removed from one side of the waveguide lack of strain symmetry in both vertical and horizontal directions could further distort the silicon lattice centro-symmetry and induce higher $\chi^{(2)}{ }_{x x y}$. In such a case FOM could be modified to:

$$
F O M=\frac{\iint_{S i}\left(c_{1} \frac{\partial \varepsilon_{x x}}{\partial y}+c_{2} \frac{\partial \varepsilon_{y y}}{\partial x}\right)|E|^{2} d x d y}{\int_{-\infty}^{\infty} \int_{-\infty}^{\infty}|E|^{2} d x d y}
$$

where $\mathrm{c}_{1}$ and $\mathrm{c}_{2}$ proportionality constants relating $\partial \varepsilon_{\mathrm{yy}} / \partial \mathrm{x}$ and $\partial \varepsilon_{\mathrm{xx}} / \partial \mathrm{y}$ to the local change in refractive index for a given electric field, which need to measured experimentally. 2D centro-symmetric crystal model explained in section 2.4 and Appendix C indicates $\partial \varepsilon_{y y} / \partial x$ induces a change in $x$ component of $E_{n e t}$. Therefore another component of second order nonlinearity is induced, namely $\chi_{\text {xyx }}^{(2)}$. Measurements need to be carried out to 
characterize the strength of this component, which can be higher or lower than $\chi^{(2)}{ }_{x x y}$ currently reported in the literature. Exploiting $\chi_{\text {xyx }}^{(2)}$ requires the electric modulation field to be parallel to $x$ direction, so a change in electrode configuration is needed.

Strain component $\varepsilon_{\mathrm{yy}}$ and its gradient in $x$ direction $\partial \varepsilon_{\mathrm{yy}} / \partial x$ for a silicon waveguide with straining layer deposited only on one side is illustrated in Figure 6.1 (a) and (b), respectively.

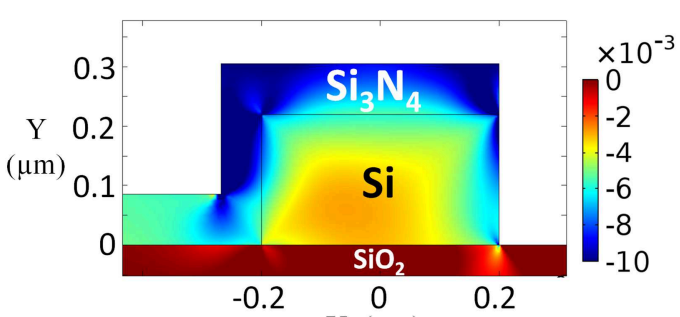

(a)

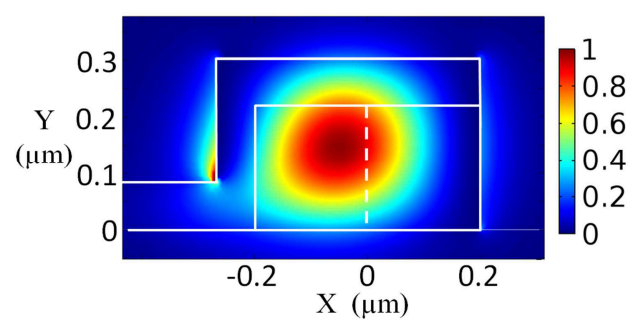

(c)

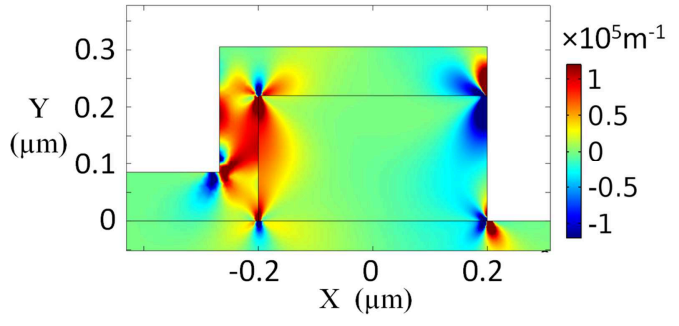

(b)

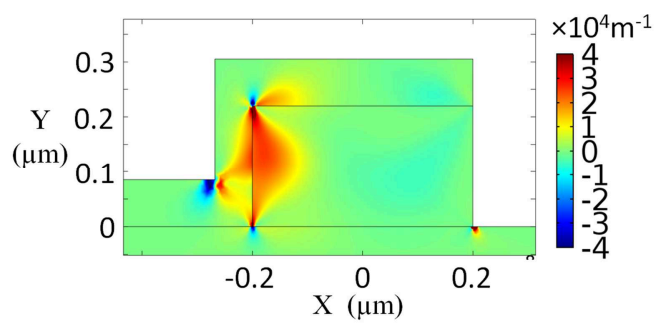

(d)

Figure 6.1 Strain induced effect for waveguide structures with horizontal asymmetric deposition of a-Si straining layer.

(a) Normalized mode intensity distribution. The dotted line marks the middle section of the waveguide core. (b) Strain component $\varepsilon_{\mathrm{yy}}$. (c) Strain gradient $\partial \varepsilon_{\mathrm{yy}} / \partial \mathrm{y}$. (d) Overlap of the normalized mode intensity and strain gradient.

If the straining material has a refractive index similar to silicon, amorphous silicon for instance, in addition to asymmetry in strain gradient distribution the optical mode shifts horizontally to one side of the waveguide core, as shown in Figure 6.1 (c). Therefore the 
mode "sees" only parts of strain gradient, as illustrated in Figure 6.1 (d) which is expected to contribute to induced $\chi^{(2)}$.

The main focus of this study was to improve effective $\chi^{(2)}$ in silicon waveguides, however for a practical modulator device other performance measures such as $V_{\pi} L$, end to end loss and extinction ratio as discussed section 1.6 should also be addressed. A future study could focus on these issues.

In conclusion the stress-induced Pockels effect shows promising prospects for the realization of silicon-based linear electro-optical modulators with performance approaching the state-of-the-art $\mathrm{LiNbO}_{3}$ modulator technology.

\subsection{Achievements and contributions}

During the course of my Master's degree I was fortunate enough to present my work during Photonics North 2012, 2013 and Group IV Photonics 2013 conferences. I was awarded for the best student presentation in Photonics Theory, Design and Simulation session of Photonics North 2013. I have also been a contributing author to one journal paper. The following is the list of all papers for which I have been either the first or a contributing author:

1. A. Aleali, D.-X. Xu, J. H. Schmid, P. Cheben, and W. N. Ye, "Optimization of stressinduced Pockels effect in silicon waveguides for optical modulators," in Group IV Photonics, 10th International Conference on, 2013, vol. 3, no. 2, pp. 109-110.

2. A. Aleali, D.-X. Xu, J. H. Schmid, P. Cheben and W. N. Ye, "Stress-Induced Pockels Effect in Silicon Modulators," SPIE Photonics North'13, Jun 2013.

3. M. Ibrahim, J. H. Schmid, A. Aleali, P. Cheben, J. Lapointe, S. Janz, P. J. Bock, A. Densmore, B. Lamontagne, R. Ma, D.-X. Xu, and W. N. Ye, "Athermal silicon 
waveguides with bridged subwavelength gratings for TE and TM polarizations", Optics Express 20 (16) 18356-18361 (2012)”

4. M.Ibrahim, A. Aleali, J.H. Schmid, P.Cheben, J. Lapointe, S. Janz, P.J. Bock, A. Densmore, B. Lamontagne, R. Ma, D.-X. Xu, and W.N. Ye, "Temperatureindependent silicon waveguides comprising bridged subwavelength gratings", Proc. SPIE 8412, SPIE Photonics North'12, Jun 2012.

Currently two journal papers are being prepared for submission:

1. A. Aleali, J. H. Schmid, D.-X. Xu, P. Cheben, and W. N. Ye, "Optimization of straininduced Pockels effect in silicon waveguides for optical modulators," Optics Express, (2014).

2. A. Aleali, J. H. Schmid, D.-X. Xu, P. Cheben, and W. N. Ye, "Enhancement of Strain Induced Pockels Effect in Silicon Waveguides," Optics Letters, (2014). 


\section{Appendices}

\section{Appendix A Geometrical parameters for waveguides used in Figure 4.3 plot that}

correlates FOM and effective $\chi^{(2)}$

\begin{tabular}{|c|c|c|c|c|c|c|}
\hline Ref. & Type & $\begin{array}{c}\text { Stress in } \mathrm{Si}_{3} \mathrm{~N}_{4} \\
\text { layer }(\mathrm{GPa})\end{array}$ & $\mathrm{w}(\mu \mathrm{m})$ & $\mathrm{h}(\mu \mathrm{m})$ & $\begin{array}{c}\text { Effective } \\
\chi^{(2)} \\
(\mathrm{pm} / \mathrm{V})\end{array}$ & $\begin{array}{c}\mathrm{FOM} \\
\left(\mathrm{m}^{-1}\right)\end{array}$ \\
\hline$[14]$ & Channel & +1.2 & 2.3 & 2.1 & $20 \pm 15$ & -946 \\
\hline$[14]$ & Channel & +1.2 & 2.5 & 2.0 & $20 \pm 15$ & -993 \\
\hline$[14]$ & Channel & +1.2 & 10.7 & 2.0 & $40 \pm 30$ & -317 \\
\hline$[14]$ & Channel & -0.5 & 2.0 & 2.5 & $11 \pm 8$ & 351 \\
\hline$[14]$ & Channel & -0.5 & 2.5 & 2.3 & $8 \pm 5$ & 364 \\
\hline$[14]$ & Channel & -0.5 & 11.7 & 2.5 & $4 \pm 3$ & 498 \\
\hline$[14]$ & Channel & -0.06 & 2.0 & 2.2 & $9 \pm 6$ & 378 \\
\hline$[14]$ & Channel & -0.06 & 2.3 & 2.2 & $10 \pm 7$ & 499 \\
\hline$[14]$ & Channel & -0.06 & 11.7 & 2.2 & $14 \pm 10$ & 182 \\
\hline$[13]$ & Ridge & +1 & 0.5 & 0.22 & 50 & 3660 \\
\hline$[13]$ & Ridge & +1 & 0.45 & 0.22 & 56 & 4800 \\
\hline$[13]$ & Ridge & +1 & 0.4 & 0.22 & 65 & 5700 \\
\hline$[13]$ & Ridge & +1 & 0.35 & 0.22 & 69 & 6130 \\
\hline$[13]$ & Ridge & +1 & 0.3 & 0.22 & 79 & 5810 \\
\hline
\end{tabular}

Table A. 1 Geometrical parameters for waveguides used in Figure 4.3 plot that correlates calculated FOM and experimentally measured effective $\chi^{(2)}$. 


\section{Appendix B Experimental procedure}

In order to experimentally demonstrate a-Si devices discussed in Chapter 5, Mach Zehnder Interferometer (MZI) modulators were designed. Due to complications in the fabrication process, the samples were not ready at the time of this thesis defense. Here we provide an example of an MZI device designed as well as a plan for the experimental setup that can be used in the future to verify results predicated in this thesis.

\section{B.1 Mach-Zehnder Interferometer schematic}

An example of MZI in the mask outline in shown in Figure B-1 (a). The MZI is comprised of the following sections:

- Inverted input edge coupler in Figure B-1 (b): It is an inverse tapered waveguide with narrow end being $100 \mathrm{~nm}$ wide which faces the edge of the wafer where light from the laser is coupled into the MZI. The taper length is $\sim 1.1 \mathrm{~mm}$. The high ratio of width/length ensures the mode gradually evolves to match the mode of the MZI.

- Y-junction Figure B-1 (c): the end of the coupler is connected to a Y-junction that splits the input light into two equal portions.

- Arms: The two arms are channel waveguides that are $200 \mathrm{~nm}$ to $500 \mathrm{~nm}$ wide. There is a $46 \mu \mathrm{m}$ length difference introduced in top arm.

- Push-pull electrodes Figure B-1 (d): Electrodes are made of a gold and titanium alloy that is deposited on top of the $\mathrm{SiO}_{2}$ spacing layer. The push-pull configuration applies opposite polarity of the $E_{m o d}$ in each arm of the MZI, which improves the $\mathrm{V}_{\pi} \mathrm{L}$ two folds. 


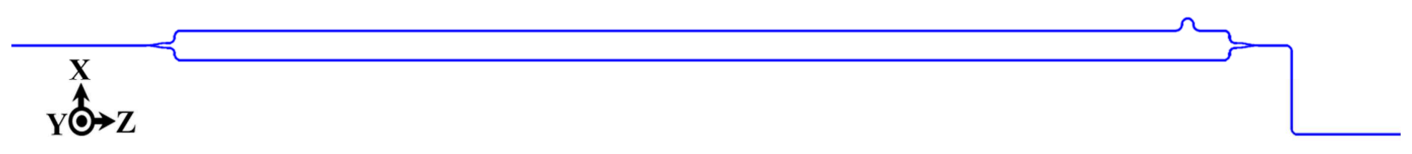

(a)

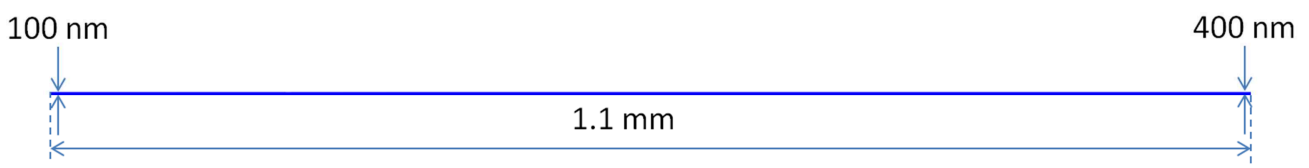

(b)

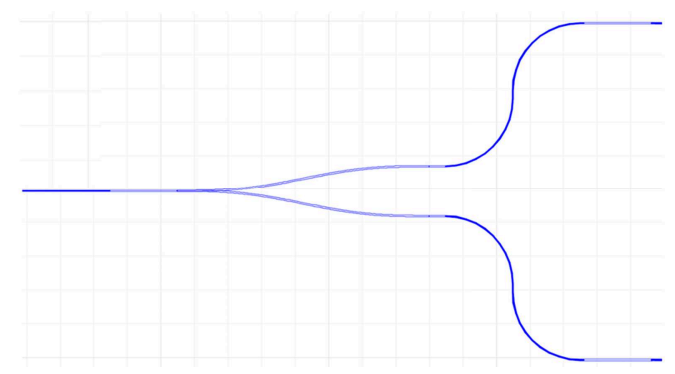

(c)

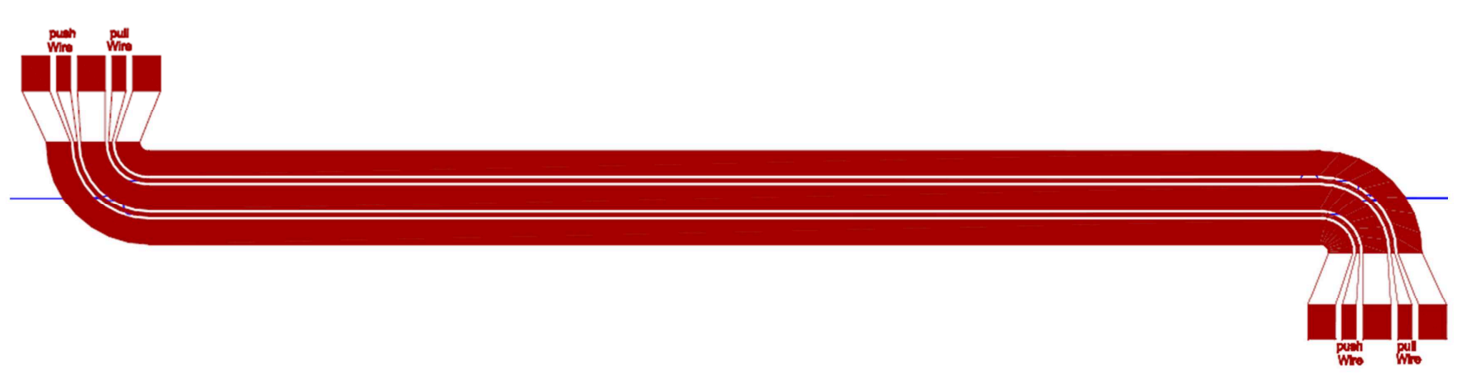

(d)

Figure B-1 Example of MZI in the mask outline.

(a): MZI. (c) Inverse Taper (b) Push-pull Ti/Au electrodes. (c) Y-junction. (d) Push-pull electrodes.

An important design consideration for waveguides with bends, such as the Y-junction shown above, is the minimum arc radius at which the loss is negligible. We used RSoft $®$ to calculate effective index $\left(\mathrm{n}_{\mathrm{eff}}\right)$ of waveguides with various bend radii. Then, the imaginary part of the $\mathrm{n}_{\mathrm{eff}}$ is converted to loss in decibel using:

$$
\operatorname{loss}=10 \log \left(\mathrm{e}^{-4 \pi \alpha \lambda}\right)
$$


where $\alpha$ is the imaginary part of the refractive index. In Figure B-2 we see that for bend radius of $\sim 7 \mu \mathrm{m}$ or loss is negligible. We used minimum bend radius of $10 \mu \mathrm{m}$ to allow some tolerance for fabrication imperfections.

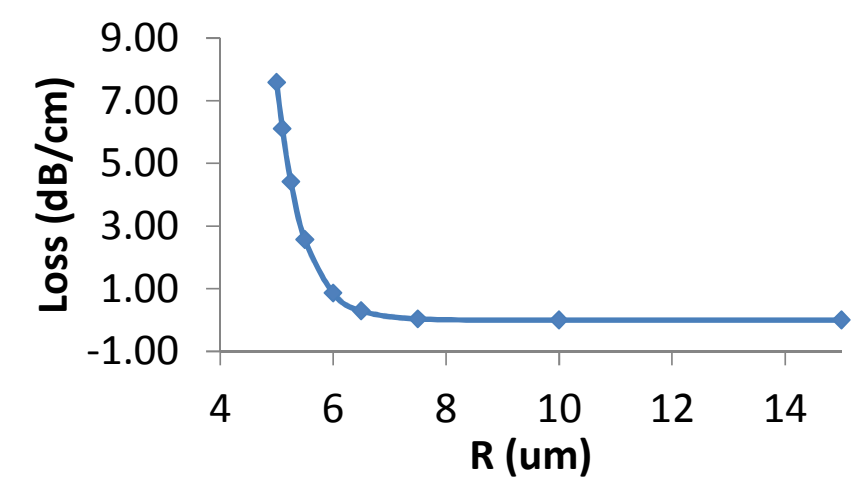

Figure B-2 Bend loss for a $300 \mathrm{~nm}$ wide waveguide on $220 \mathrm{~nm}$ SOI and $100 \mathrm{~nm}$ cladding thickness. $R$ is arc radius.

\section{B.2 Experimental Setup}

The electro-optic characterization of the fabricated modulators can be carried out using the following experimental setup:

1. At the input of the modulator a tunable laser is used to lunch beam light around $\lambda=1550 \mathrm{~nm}$ into the input coupler of an MZI.

2. At the output of the modulator a photo detector registers the output spectrum. Spectrum needs to be obtained at two state, one when there is no applied voltage to the electrodes and another when a voltage is applied; an example is shown in Figure B-3. The spectral shift $(\Delta \lambda)$ between the two spectrums shows the strength of electro-optic effect. 


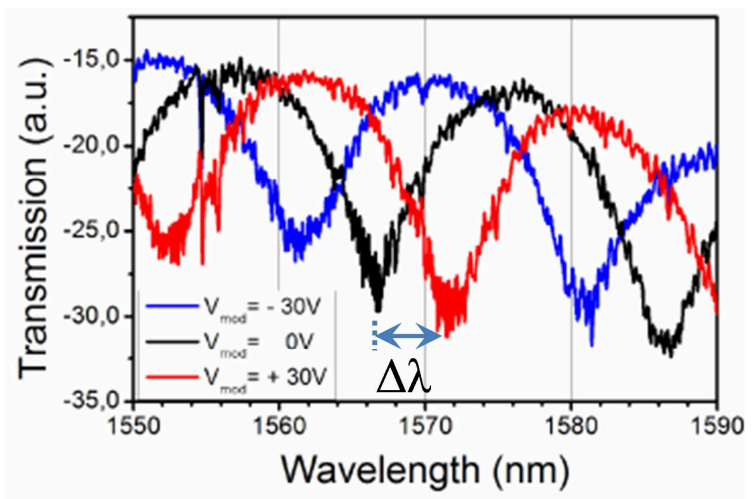

Figure B-3 Sample spectrum showing spectral shift from experiments carried out by Chmielak et al. [13].

3. To extract effective $\chi^{(2)}$ from $\Delta \lambda$ we can use the following equation to first calculate $\Delta \mathrm{n}_{\text {eff: }}: \Delta \lambda \Delta n_{\text {eff }}=n_{\text {eff }} \lambda$ The change in the effective index $\left(\Delta \mathrm{n}_{\text {eff }}\right)$ of the waveguide can be converted to $\chi_{\text {eff }}^{(2)}$ using: $\chi_{e f f}^{(2)}=n_{e f f} \cdot \Delta n_{\text {eff }} / E_{\text {Mod }}$ where, $E_{\text {Mod }}$ is the electric modulation field inside the silicon waveguide which is determined using numerical simulation.

\section{Appendix C Derivation of electric field due to 2D centro-symmetric lattice}

In this section we derive equation 2.5 that expresses net electric field at the center of a 2 dimensional centro-symmetric lattice. From Coulomb's law we know that electric field due to small charge $d Q$ is

$$
d \vec{E}=\frac{k d Q}{|\vec{r}|^{2}} \hat{r}
$$

where in SI system $k=\frac{1}{4 \pi \varepsilon_{0}}, d Q$ is charge in Coulombs $\vec{r}$ is a vector from source of charge pointing towards the point of interest, and $\hat{r}$ is the unit vector for $\vec{r}$. 


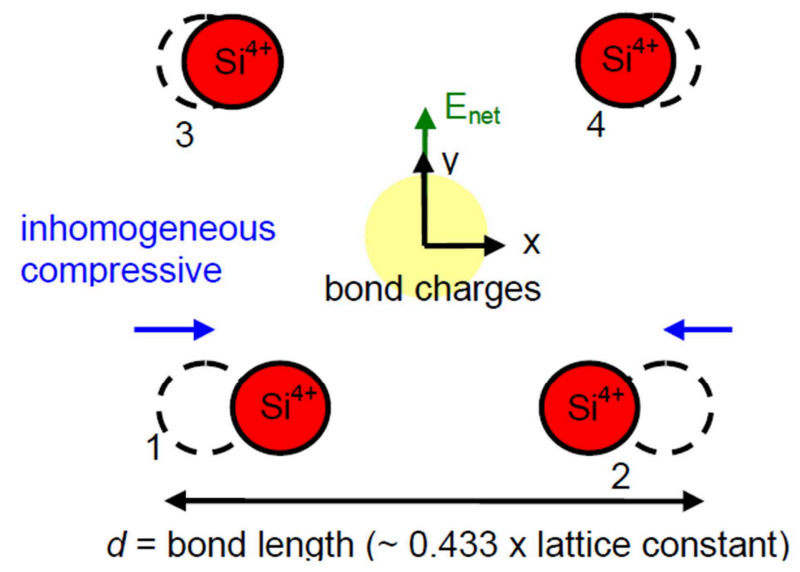

(a)

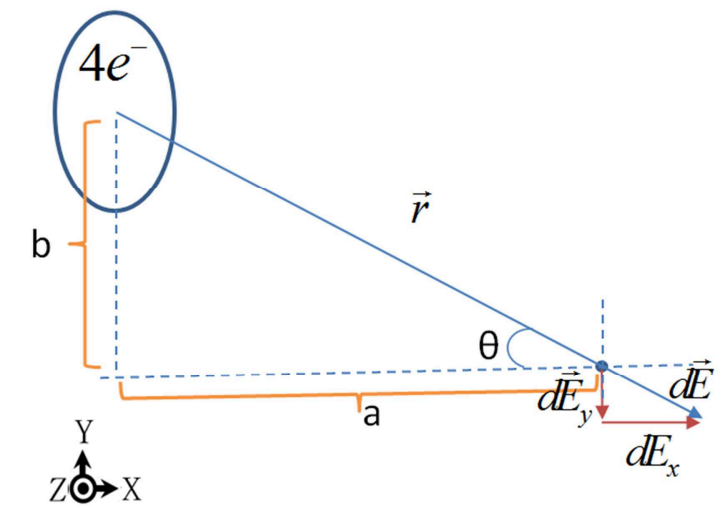

Figure C.4 Evaluation of $\mathrm{E}$ field due to (a) a inhomogeneously strained 2D centrosymmetric lattice and (b) a single silicon atom.

Since there is a symmetry in $x$ direction, at the center of the lattice of the lattice $d \vec{E}_{x}$ due to atom 1 and 3 cancels $d \vec{E}_{x}$ due to 2 and 4 , respectively. Since the bottom atoms are moved from their centro-symmetric position, $d \vec{E}_{y}$ due to atoms 1 and 2 , do not completely cancel $d \vec{E}_{y}$ due to atoms 3 and 4 . The $d \vec{E}_{y}$ due to atom 3 (Figure C.3 (b)):

$$
d E_{y}=\frac{k d Q}{|\vec{r}|^{2}} \sin \theta
$$


Upon inspection we can see that

$$
|\vec{r}|=\sqrt{a^{2}+b^{2}}
$$

Since $\sin \theta=\frac{b}{\sqrt{a^{2}+b^{2}}}$ we have:

$$
d E_{y}=\frac{k d Q b}{\left(\mathrm{a}^{2}+b^{2}\right)^{3 / 2}}
$$

In silicon atoms outer most energy level has 4 electrons so $d Q=4 e^{-}$where $e^{-}$is charge of an electron. In our lattice along $y$ axis for atoms 3 and $4 a=d / 2$ and $b=d / 2-y$. So the electric field component due to this silicon atom is:

$$
E_{y}=\frac{e}{\pi \varepsilon_{0}} \frac{\left(\frac{\mathrm{d}}{2}-y\right)}{\left(\left(\frac{\mathrm{d}}{2}\right)^{2}+\left(\frac{\mathrm{d}}{2}-y\right)^{2}\right)^{3 / 2}}
$$

For atoms 1 and 2 at bottom of the lattice $b=d / 2+y$ and $a=\frac{d}{2}+\nabla S d \frac{d}{2}$ where $\nabla S=\frac{\partial \varepsilon_{x x}}{\partial y}$ (strain gradient). Adding contributions from all four atoms we have:

$$
E_{y}=\frac{2 e}{\pi \varepsilon_{0}}\left(\frac{\left(\frac{\mathrm{d}}{2}-y\right)}{\left(\left(\frac{\mathrm{d}}{2}\right)^{2}+\left(\frac{\mathrm{d}}{2}-y\right)^{2}\right)^{3 / 2}}+\frac{\left(\frac{\mathrm{d}}{2}+y\right)}{\left(\left(\frac{\mathrm{d}}{2}+\nabla S d \frac{\mathrm{d}}{2}\right)^{2}+\left(\frac{\mathrm{d}}{2}+y\right)^{2}\right)^{3 / 2}}\right)
$$

\section{Appendix D MATLAB code for the Comsol® program}

Below is the MATLAB code for a sample Comsol ${ }^{\circledR}$ file used to simulate the strain gradient distribution and the optical mode for a SiGe strained waveguide. 
function out $=$ model

$\%$

$\%$ SiGeChannelSlot.m

$\%$

\% Model exported on Apr 1 2014, 12:24 by COMSOL 4.4.0.150.

import com.comsol.model.*

import com.comsol.model.util.*

model $=$ ModelUtil.create('Model');

model.modelPath('W:IComsol filesl6. Results 15-DeclNanolSiGe');

model.name('SiGe Channel Slot Depth ScanW 450 nm CT 85 nm.mph');

model.param.set('nSi', '3.476', 'Refractive index, silicon (Si)');

model.param.set('nSiO2', '1.444', 'Refractive index, silica ( $\mathrm{SiO} 2)$ ');

model.param.set('alphaSi', '3.6e-6[1/K]', 'Thermal expansion coefficient, silicon');

model.param.set('alphaSiO2', '0.54e-6[1/K]', 'Thermal expansion coefficient, silica');

model.param.set('ESi', '130[GPa]', 'Young"s modulus, silicon');

model.param.set('ESiO2', '76.734[GPa]', 'Young"s modulus, silica');

model.param.set('nuSi', '0.27', 'Poisson"s ratio, silicon');

model.param.set('nuSiO2', '0.186', 'Poisson"s ratio, silica');

model.param.set('rhoSi', '2330[kg/m^3]', 'Density, silicon');

model.param.set('rhoSiO2', '2203[kg/m^3]', 'Density, silica');

model.param.set('B1_SiO2', '0.65e-12[m^2/N]', 'First stress optical coefficient');

model.param.set('B2_SiO2', '4.2e-12[m²/N]', 'Second stress optical coefficient');

model.param.set('T1', '20[degC]', 'Operating temperature');

model.param.set('T0', '1000[degC]', 'Reference temperature');

model.param.set('lambda0_emw', '1.55[um]', 'Free space wavelength');

model.param.set('w1', '450e-9', 'Width of ridge');

model.param.set('t1', '85e-9', 'Thickness of the upper cladding');

model.param.set('D1', 'H1', 'Etch depth');

model.param.set('L1', '300e-6', 'Length of the system');

model.param.set('t2', 'H1-D1', 'Thickness of the lateral Si');

model.param.set('H1', '220e-9', 'Height of the ridge');

model.param.set('EGe', '103 [GPa]', 'Younge Modulud for Ge');

model.param.set('rhoSiGe', '2.93 [g/cm^3]', 'Density of SiGe 0.8, from Palik');

model.param.set('alphaSiGe', 'p*alphaSi+(1-p)*alphaGe', 'Thermal expanssion coefficient of

SiGe');

model.param.set('ESiSub', 'ESi*1000', 'Young Modulus for Substrate');

model.param.set('intrnsx_SiGe', '-1000e6', 'Intrinsic stress of SiGe layer');

model.param.set('B1_Si', '-1.749e-11 [m^2/N]', '1st stress-optic coefficient of Si');

model.param.set('B2_Si', '5.6285e-12 [m^2/N]', '2st stress-optic coefficient of Si');

model.param.set('slw1', '60e-9', 'Slot Width');

model.param.set('sld1', '20e-9', 'Slot Depth');

model.param.set('slh1', 'H1-sld1', 'Slot Height');

model.param.set('p', '0.8', 'Si content in SiGe');

model.param.set('nGe', '4.275', 'Refractive index of Ge');

model.param.set('kGe', '5.67e-3', 'Extinction coefficient of Ge');

model.param.set('alphaGe', '5.8e-6', 'TEC of Ge');

model.param.set('nuGe', '0.25', 'Poisson ratio of Ge');

model.param.set('ESiGe', 'p*ESi+(1-p)*EGe', 'Young"s modulus of SiGe');

model.param.set('nSiGe', 'p*nSi+(1-p)*nGe', 'Refractive index of $\left.\mathrm{SiGe}^{\prime}\right)$;

model.param.set('kSi'. '0'. 'Extinction coefficient of Si'): 
model.param.set('kSiGe', '(1-p)*kGe', 'Extinction coefficient of SiGe');

model.param.set('nuSiGe', 'p*nuSi+(1-p)*nuGe', 'Poisson ratio of SiGe');

model.modelNode.create('mod1');

model.modelNode('mod1').name('Model 1');

model.param.set('kSiGe', '(1-p)*kGe', 'Extinction coefficient of SiGe');

model.param.set('nuSiGe', 'p*nuSi+(1-p)*nuGe', 'Poisson ratio of SiGe');

model.modelNode.create('mod1');

model.modelNode('mod1').name('Model 1');

model.geom.create('geom1', 2);

model.geom('geom1').feature.create('r1', 'Rectangle');

model.geom('geom1').feature.create('b3', 'BezierPolygon');

model.geom('geom1').feature.create('b4', 'BezierPolygon');

model.geom('geom1').feature.create('r5', 'Rectangle');

model.geom('geom1').feature.create('r6', 'Rectangle');

model.geom('geom1').feature('r1').set('size', \{'L1' '6e-6'\});

model.geom('geom1').feature('r1').set('pos', \{'-L1/2' '-9e-6'\});

model.geom('geom1').feature('b3').set('degree', \{'1' '1' '1' '1' '1' '1' '1' '1'\});

model.geom('geom1').feature('b3').set('p', \{'-L1/2' '-L1/2' '-w1/2-0.8*t1' '-w1/2-0.8*t1'

'w1/2+0.8*t1' 'w1/2+0.8*t1' 'L1/2' 'L1/2' '-L1/2'; 't2' 't1+t2' 't1+t2' 't1+H1' 't1+D1+t2' 't1+t2'

't1+t2' '0' '0'\});

model.geom('geom1').feature('b3').set('w', \{'1' '1' '1' '1' '1' '1' '1' '1' '1' '1' ...'1' '1' '1' '1' '1' '1'\});

model.geom('geom1').feature('b4').set('degree', \{'1' '1' '1' '1' '1' '1' '1' '1' '1' '1' ... '1' '1'\});

model.geom('geom1').feature('b4').set('p', \{'-L1/2' '-L1/2' '-w1/2' '-w1/2' '-slw1/2' '-slw1/2'

'slw1/2' 'slw1/2' 'w1/2' 'w1/2' 'L1/2' 'L1/2' '-L1/2'; '0' 't2' 't2' 'H1' 'H1' 'H1-sld1' 'H1-sld1' 'H1'

'H1' 't2' 't2' '0' '0'\});

model.geom('geom1').feature('b4').set('w', \{'1' '1' '1' '1' '1' '1' '1' '1' '1' '1' ... '1' '1' '1' '1' '1' '1'

'1' '1' '1' '1' ... '1' '1' '1' '1'\});

model.geom('geom1').feature('r5').set('size', \{'L1' '3e-6'\});

model.geom('geom1').feature('r5').set('pos', \{'-L1/2' '-3e-6'\});

model.geom('geom1').feature('r6').set('size', \{'8000e-9' '3000e-9' $\}$ );

model.geom('geom1').feature('r6').set('pos', \{'-4000e-9' '-1e-6'\});

model.geom('geom1').run;

model.variable.create('var1');

model.variable('var1').model('mod1');

model.variable('var1').set('ez', 'para*(e0+(e1*X+e $2 * \mathrm{Y}) / 1[\mathrm{~m}])$ ', 'Out-of-plain strain, z

compnent');

model.variable('var1').set('sx', 'solid.S111+solid.D13*ez', 'Stress, x component');

model.variable('var1').set('sy', 'solid.S122+solid.D23*ez', 'Stress, y component');

model.variable('var1').set('sz', 'solid.S133+solid.D33*ez', 'Stress, z component');

model.variable('var1').set('wc1', '-

(solid.D13*ez*test(solid.el11)+solid.D23*ez*test(solid.el22)+sz*test(ez))*solid.d', 'Weak contrinbution due to out-of-plain strain');

model.variable.create('var2');

model.variable('var2').model('mod1');

model.variable('var2').set('N', 'nSiGe');

model.variable('var2').set('k', 'kSiGe'); 
model.variable('var2').selection.geom('geom1', 2);

model.variable('var2').selection.set([5]);

model.variable.create('var3');

model.variable('var3').model('mod1');

model.variable('var3').set('N', 'nSi');

model.variable('var3').set('k', '0');

model.variable('var3').selection.geom('geom1', 2);

model.variable('var3').selection.set([7]);

model.variable.create('var4');

model.variable('var4').model('mod1');

model.variable('var4').set('N', 'nSiO2');

model.variable('var4').set('k', '0');

model.variable('var4').selection.geom('geom1', 2);

model.variable('var4').selection.set([4]);

model.variable.create('var5');

model.variable('var5').model('mod1');

model.variable('var5').set('Nx', 'N-(B1_Si*solid.sx+B2_Si*(solid.sy+solid.sz))', 'Refractive index, $\mathrm{x}$ component');

model.variable('var5').set('Ny', 'N-(B1_Si*solid.sy+B2_Si*(solid.sx+solid.sz))', 'Refractive index, y component');

model.variable('var5').set('Nz', 'N-(B1_Si*solid.sz+B2_Si*(solid.sx+solid.sy))', 'Refractive

index, z component');

model.variable('var5').selection.geom('geom1', 2);

model.variable('var5').selection.set([5 7]);

model.variable.create('var6');

model.variable('var6').model('mod1');

model.variable('var6').set('Nx', 'N-(B1_SiO2*solid.sx+B2_SiO2*(solid.sy+solid.sz))');

model.variable('var6').set('Ny', 'N-(B1_SiO2*solid.sx+B2_SiO2*(solid.sy+solid.sz))');

model.variable('var6').set('Nz', 'N-(B1_SiO2*solid.sx+B2_SiO2*(solid.sy+solid.sz))');

model.variable('var6').selection.geom('geom1', 2);

model.variable('var6').selection.set([4]);

model.variable.create('var7');

model.variable('var7').model('mod1');

model.variable('var7').set('N', '1');

model.variable('var7').set('Nx', '1');

model.variable('var7').set('Ny', '1');

model.variable('var7').set('Nz', '1');

model.variable('var7').set('k', '0');

model.variable('var7').selection.geom('geom1', 2);

model.variable('var7').selection.set([6]);

model.material.create('mat1');

model.material('mat1').propertyGroup.create('RefractiveIndex', 'Refractive index');

model.material('mat1').selection.set([7]);

model.material.create('mat2');

model.material('mat2').propertyGroup.create('RefractiveIndex', 'Refractive index');

model.material('mat2').selection.set([2 4]);

model.material.create('mat3'); 
model.material('mat3').propertyGroup.create('RefractiveIndex', 'Refractive index'); model.material('mat3').selection.set([1]);

model.material.create('mat5');

model.material('mat5').propertyGroup('def').func.create('eta', 'Piecewise');

model.material('mat5').propertyGroup('def').func.create('Cp', 'Piecewise');

model.material('mat5').propertyGroup('def').func.create('rho', 'Analytic');

model.material('mat5').propertyGroup('def').func.create('k', 'Piecewise');

model.material('mat5').propertyGroup('def').func.create('cs', 'Analytic');

model.material('mat5').selection.set([6]);

model.material.create('mat6');

model.material('mat6').selection.set([3 5 8 $]$ );

model.physics.create('solid', 'SolidMechanics', 'geom1');

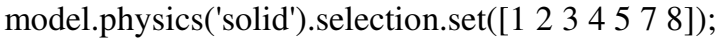

model.physics('solid').feature('lemm1').feature.create('te1', 'ThermalExpansion', 2);

model.physics('solid').feature('lemm1').feature.create('iss1', 'InitialStressandStrain', 2);

model.physics('solid').feature('lemm1').feature('iss1').selection.set([3 58 8]);

model.physics('solid').feature.create('fix1', 'Fixed', 0);

model.physics('solid').feature('fix1').selection.set([1]);

model.physics('solid').feature.create('disp1', 'Displacement0', 0);

model.physics('solid').feature('disp1').selection.set([25]);

model.physics('solid').feature.create('ge1', 'GlobalEquations', -1);

model.physics('solid').feature.create('weak1', 'WeakContribution', 2);

model.physics('solid').feature('weak1').selection.all;

model.physics.create('emw', 'ElectromagneticWaves', 'geoml');

model.physics('emw').selection.set([[4 56 6 7]);

model.mesh.create('mesh1', 'geom1');

model.mesh('mesh1').feature.create('ftri2', 'FreeTri');

model.mesh('mesh1').feature.create('ftri1', 'FreeTri');

model.mesh('mesh1').feature('ftri2').feature.create('size1', 'Size');

model.mesh('mesh1').feature('ftri2').feature('size1').selection.geom('geom1', 2);

model.mesh('mesh1').feature('ftri2').feature('size1').selection.set([7]);

model.mesh('mesh1').feature('ftri1').feature.create('size1', 'Size');

model.mesh('mesh1').feature('ftri1').feature('size1').selection.geom('geom1', 2);

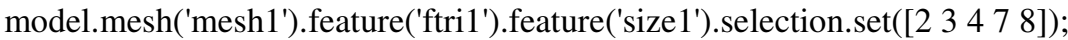

model.result.table.create('tbl1', 'Table');

model.variable('var1').active(false);

model.variable('var1').name('Variables 1a');

model.variable('var2').name('Variables 2a');

model.variable('var3').name('Variables 3a');

model.variable('var4').name('Variables 4a');

model.variable('var5').name('Variables 5a');

model.view('view1').set('locked', true);

model.view('view1').set('showlabels', true);

model.view('view1').axis.set('xmin', '-3e-7');

model.view('view1').axis.set('ymin', '-1e-7');

model.view('view1').axis.set('xmax', '3e-7');

model.view('view1').axis.set('ymax', '4e-7'); 
model.material('mat1').name('Si Device Layer');

model.material('mat1').propertyGroup('def').set('youngsmodulus', 'ESi');

model.material('mat 1').propertyGroup('def').set('poissonsratio', 'nuSi');

model.material('mat1').propertyGroup('def').set('density', 'rhoSi');

model.material('mat1').propertyGroup('def').set('thermalexpansioncoefficient', \{ 'alphaSi' '0'

'0' '0' 'alphaSi' '0' '0' '0' 'alphaSi'\});

model.material('mat1').propertyGroup('RefractiveIndex').set('n', ");

model.material('mat1').propertyGroup('RefractiveIndex').set('ki', ");

model.material('mat1').propertyGroup('RefractiveIndex').set('n', \{'nSi' '0' '0' '0' 'nSi' '0' '0' '0'

'nSi'\});

model.material('mat1').propertyGroup('RefractiveIndex').set('ki', \{ '0' '0' '0' '0' '0' '0' '0' '0'

' 0 '\});

model.material('mat2').name('BOX');

model.material('mat2').propertyGroup('def').set('youngsmodulus', 'ESiO2');

model.material('mat2').propertyGroup('def').set('poissonsratio', 'nuSiO2');

model.material('mat2').propertyGroup('def').set('density', 'rhoSiO2');

model.material('mat2').propertyGroup('def').set('thermalexpansioncoefficient', \{'alphaSiO2'

'0' '0' '0' 'alphaSiO2' '0' '0' '0' 'alphaSiO2'\});

model.material('mat2').propertyGroup('RefractiveIndex').set('n', ");

model.material('mat2').propertyGroup('RefractiveIndex').set('ki', ");

model.material('mat2').propertyGroup('RefractiveIndex').set('n', \{'nSiO2' '0' '0' '0' 'nSiO2' '0'

'0' '0' 'nSiO2'\});

model.material('mat2').propertyGroup('RefractiveIndex').set('ki', \{ '0' '0' '0' '0' '0' '0' '0' '0'

'0'\});

model.material('mat3').name('Si Substrate');

model.material('mat3').propertyGroup('def').set('youngsmodulus', 'ESiSub');

model.material('mat3').propertyGroup('def').set('density', 'rhoSi');

model.material('mat3').propertyGroup('def').set('poissonsratio', 'nuSi');

model.material('mat3').propertyGroup('def').set('thermalexpansioncoefficient', \{ 'alphaSi' '0'

'0' '0' 'alphaSi' '0' '0' '0' 'alphaSi'\});

model.material('mat3').propertyGroup('RefractiveIndex').set('n', ");

model.material('mat3').propertyGroup('RefractiveIndex').set('ki', ");

model.material('mat3').propertyGroup('RefractiveIndex').set('n', \{'nSi' '0' '0' '0' 'nSi' '0' '0' '0' 'nSi'\});

model.material('mat3').propertyGroup('RefractiveIndex').set('ki', \{ '0' '0' '0' '0' '0' '0' '0' '0'

' 0 '\});

model.material('mat5').name('Air');

model.material('mat5').propertyGroup('def').func('eta').set('pieces', \{'200.0' '1600.0' '-

8.38278E-7+8.35717342E-8* $\mathrm{T}^{\wedge} 1-7.69429583 \mathrm{E}-11^{*} \mathrm{~T}^{\wedge} 2+4.6437266 \mathrm{E}-14 * \mathrm{~T}^{\wedge} 3-$

$\left.\left.1.06585607 \mathrm{E}-17 * \mathrm{~T}^{\wedge} 4^{\prime}\right\}\right)$;

model.material('mat5').propertyGroup('def').func('eta').set('arg', 'T');

model.material('mat5').propertyGroup('def').func('Cp').set('pieces', \{ '200.0' '1600.0'

'1047.63657-0.372589265* $\mathrm{T}^{\wedge} 1+9.45304214 \mathrm{E}-4 * \mathrm{~T}^{\wedge} 2-6.02409443 \mathrm{E}-7 * \mathrm{~T}^{\wedge} 3+1.2858961 \mathrm{E}-$

$10 * \mathrm{~T}^{\wedge} 4$ ' $\left.\}\right)$ 
model.material('mat5').propertyGroup('def').func('Cp').set('arg', 'T');

model.material('mat5').propertyGroup('def').func('rho').set('args', \{'pA' 'T'\});

model.material('mat5').propertyGroup('def').func('rho').set('expr', 'pA*0.02897/8.314/T');

model.material('mat5').propertyGroup('def').func('rho').set('dermethod', 'manual');

model.material('mat5').propertyGroup('def').func('rho').set('plotargs', \{'pA' " "; 'T' " "\});

model.material('mat5').propertyGroup('def').func('rho').set('argders', \{'pA'

'd(pA*0.02897/8.314/T,pA)'; 'T' 'd(pA*0.02897/8.314/T,T)'\});

model.material('mat5').propertyGroup('def').func('k').set('pieces', \{ 200.0 ' '1600.0' '-

$0.00227583562+1.15480022 \mathrm{E}-4 * \mathrm{~T}^{\wedge} 1-7.90252856 \mathrm{E}-8 * \mathrm{~T}^{\wedge} 2+4.11702505 \mathrm{E}-11 * \mathrm{~T}^{\wedge} 3-$

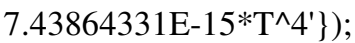

model.material('mat5').propertyGroup('def').func('k').set('arg', 'T');

model.material('mat5').propertyGroup('def').func('cs').set('args', \{'T'\});

model.material('mat5').propertyGroup('def').func('cs').set('expr', 'sqrt( $1.4 * 287 * T)$ ');

model.material('mat5').propertyGroup('def').func('cs').set('dermethod', 'manual');

model.material('mat5').propertyGroup('def').func('cs').set('plotargs', $\{$ 'T" " "\});

model.material('mat5').propertyGroup('def').func('cs').set('argders', \{ 'T' 'd( $\operatorname{sqrt}(1.4 * 287 * \mathrm{~T}), \mathrm{T}$

model.material('mat5').propertyGroup('def').set('relpermeability', \{'1' '0' '0' '0' '1' '0' '0' '0'

'1'\});

model.material('mat5').propertyGroup('def').set('relpermittivity', \{'1' '0' '0' '0' '1' '0' '0' '0' '1'\});

model.material('mat5').propertyGroup('def').set('dynamicviscosity', 'eta(T[1/K])[Pa*s]');

model.material('mat5').propertyGroup('def').set('ratioofspecificheat', '1.4');

model.material('mat5').propertyGroup('def').set('electricconductivity', \{ $0[\mathrm{~S} / \mathrm{m}]$ ' ' 0 ' ' 0 ' ' 0 '

'0[S/m]' '0' '0' '0' '0[S/m]'\});

model.material('mat5').propertyGroup('def').set('heatcapacity', 'Cp(T[1/K])[J/(kg*K)]');

model.material('mat5').propertyGroup('def').set('density', 'rho(pA[1/Pa],T[1/K]) $\left.\left[\mathrm{kg} / \mathrm{m}^{\wedge} 3\right]^{\prime}\right)$;

model.material('mat5').propertyGroup('def').set('thermalconductivity',

$\{$ 'k(T[1/K])[W/(m*K)]' '0' '0' '0' 'k(T[1/K])[W/(m*K)]' '0' '0' '0' 'k(T[1/K])[W/(m*K)]'\});

model.material('mat5').propertyGroup('def').set('soundspeed', 'cs(T[1/K])[m/s]');

model.material('mat5').propertyGroup('def').addInput('temperature');

model.material('mat5').propertyGroup('def').addInput('pressure');

model.material('mat6').name('SiGe');

model.material('mat6').propertyGroup('def').set('youngsmodulus', 'ESiGe');

model.material('mat6').propertyGroup('def').set('poissonsratio', 'nuSiGe');

model.material('mat6').propertyGroup('def').set('thermalexpansioncoefficient', \{ 'alphaSiGe'

'0' '0' '0' 'alphaSiGe' '0' '0' '0' 'alphaSiGe'\});

model.material('mat6').propertyGroup('def').set('density', 'rhoSiGe');

model.physics('solid').feature('lemm1').set('minput_strainreferencetemperature', '0');

model.physics('solid').feature('lemm1').feature('te1').set('minput_strainreferencetemperature',

' 0 ');

model.physics('solid').feature('lemm1').feature('te1').set('minput_temperature', 'T1');

)$\left.^{\prime}\right\}$ );

model.physics('solid').feature('lemm1').feature('te1').set('Tref', 'T0');

model.physics('solid').feature('lemm1').feature('iss1').set('Sil', \{'intrnsx_SiGe'; '0'; '0'; '0'; '0';

'0'; '0'; '0'; '0'\});

model.physics('solid').feature('disp1').set('Direction', \{ 0 '; '1'; '0'\});

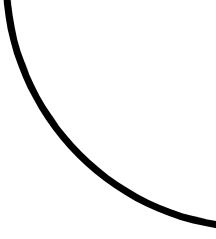


model.physics('solid').feature('ge1').set('equation', \{'e0*(1-para)'; 'e1*(1-para)'; 'e2*(1para)'\});

model.physics('solid').feature('ge1').set('initialValueU', \{'0'; '0'; '0'\});

model.physics('solid').feature('ge1').set('description', \{"; "; "\});

model.physics('solid').feature('ge1').set('initialValueUt', \{'0'; '0'; '0' $\}$ );

model.physics('solid').feature('ge1').set('name', \{'e0'; 'e1'; 'e2'\});

model.physics('solid').feature('ge 1').active(false);

model.physics('solid').feature('weak1').set('weakExpression', 'wc1');

model.physics('solid').feature('weak1').active(false);

model.physics('emw').prop('EquationForm').set('freq', '1[kHz]');

model.physics('emw').feature('wee1').set('ki', \{'k'; '0'; '0'; '0'; 'k'; '0'; '0'; '0'; 'k'\});

model.physics('emw').feature('wee1').set('n_mat', 'userdef');

model.physics('emw').feature('wee1').set('DisplacementFieldModel', 'RefractiveIndex');

model.physics('emw').feature('wee1').set('minput_strainreferencetemperature', '0');

model.physics('emw').feature('wee1').set('ki_mat', 'userdef');

model.physics('emw').feature('wee1').set('n', \{'Nx'; '0'; '0'; '0'; 'Ny'; '0'; '0'; '0'; 'Nz'\});

model.physics('emw').feature('wee1').set('editModelInputs', '1');

model.mesh('mesh1').feature('size').set('hauto', 1);

model.mesh('mesh1').feature('ftri2').feature('size1').set('hauto', 1);

model.mesh('mesh1').feature('ftri2').feature('size1').set('custom', 'on');

model.mesh('mesh1').feature('ftri2').feature('size1').set('hmaxactive', true);

model.mesh('mesh1').feature('ftri2').feature('size1').set('hmin', '4.0E-9');

model.mesh('mesh1').feature('ftri2').feature('size1').set('hmax', '1E-8');

model.mesh('mesh1').feature('ftri2').feature('size1').set('hminactive', false);

model.mesh('mesh1').feature('ftri1').feature('size1').set('hauto', 1);

model.mesh('mesh1').run;

model.result.table('tbl1').comments('Surface Integration 1 (d(solid.eX,Y)*abs(emw.Ex)^2)');

model.study.create('std1');

model.study('std1').feature.create('param', 'Parametric');

model.study('std1').feature.create('stat', 'Stationary');

model.study('std1').feature.create('mode', 'ModeAnalysis');

model.sol.create('sol1');

model.sol('sol1').study('std1');

model.sol.create('sol2');

model.sol('sol2').study('std1');

model.sol('sol2').attach('std1');

model.sol('sol2').feature.create('st1', 'StudyStep');

model.sol('sol2').feature.create('v1', 'Variables');

model.sol('sol2').feature.create('s1', 'Stationary');

model.sol('sol2').feature.create('su1', 'StoreSolution');

model.sol('sol2').feature.create('st2', 'StudyStep');

model.sol('sol2').feature.create('v2', 'Variables');

model.sol('sol2').feature.create('e1', 'Eigenvalue');

model.sol('sol2').feature('s1').feature.create('fc1', 'FullyCoupled');

model.sol('sol2').feature('s1').feature.remove('fcDef'); 
model.study('std1').feature('stat').set('initstudyhide', 'on'); model.study('std1').feature('stat').set('initsolhide', 'on');

model.study('std1').feature('stat').set('notstudyhide', 'on'); model.study('std1').feature('stat').set('notsolhide', 'on'); model.study('std1').feature('mode').set('initstudyhide', 'on'); model.study('std1').feature('mode').set('initsolhide', 'on'); model.study('std1').feature('mode').set('notstudyhide', 'on'); model.study('std1').feature('mode').set('notsolhide', 'on'); model.sol.create('sol4');

model.sol('sol4').study('std1');

model.batch.create('p1', 'Parametric');

model.batch.create('p2', 'Parametric'); model.batch.create('p3', 'Parametric');

model.batch.create('p4', 'Parametric');

model.batch('p1').feature.create('so1', 'Solutionseq'); model.batch('p2').feature.create('so1', 'Solutionseq'); model.batch('p3').feature.create('so1', 'Solutionseq'); model.batch('p4').feature.create('so1', 'Solutionseq'); model.batch('p1').study('std1'); model.batch('p2').study('std1'); model.batch('p3').study('std1'); model.batch('p4').study('std1'); model.result.numerical.create('int1', 'IntSurface'); model.result.numerical.create('int2', 'IntSurface'); model.result.numerical.create('int3', 'IntSurface'); model.result.numerical('int1').selection.set([7]); model.result.numerical('int1').set('probetag', 'none'); model.result.numerical('int2').selection.set([5]); model.result.numerical('int2').set('probetag', 'none'); model.result.numerical('int3').selection.set([4 56 6 6 ); model.result.numerical('int3').set('probetag', 'none'); model.result.create('pg1', 'PlotGroup2D'); model.result.create('pg2', 'PlotGroup2D'); model.result.create('pg3', 'PlotGroup2D'); model.result('pg1').feature.create('surf1', 'Surface'); model.result('pg1').feature('surf1').feature.create('def', 'Deform'); model.result('pg2').feature.create('surf1', 'Surface'); model.result('pg3').feature.create('surf1', 'Surface'); model.result('pg3').feature('surf1').feature.create('def', 'Deform'); model.study('std1').feature('param').set('pname', \{'sld1'\}); model.study('std1').feature('param').set('plistarr', \{'20e-9 50e-9 75e-9 85e-9 100e-9 125e-9 150e-9 200e-9'\});

model.study('std1').feature('stat').set('activate', \{'solid' 'on' 'emw' 'off'\}); model.study('std1').feature('mode').set('modeFreq', 'c_const/lambda0_emw'); model.study('std1').feature('mode').set('shift', '3'); model.study('std1').feature('mode').set('neigs', '30'); 
model.sol('sol2').attach('std1');

model.sol('sol2').feature('st1').name('Compile Equations: Stationary');

model.sol('sol2').feature('st1').set('studystep', 'stat');

model.sol('sol2').feature('v1').set('control', 'stat');

model.sol('sol2').feature('v1').feature('mod1_E').set('solvefor', false);

model.sol('sol2').feature('s1').set('control', 'stat');

model.sol('sol2').feature('st2').name('Compile Equations: Mode Analysis (2)');

model.sol('sol2').feature('st2').set('studystep', 'mode');

model.sol('sol2').feature('v2').set('initsol', 'sol2');

model.sol('sol2').feature('v2').set('notsolmethod', 'sol');

model.sol('sol2').feature('v2').set('control', 'mode');

model.sol('sol2').feature('v2').set('initmethod', 'sol');

model.sol('sol2').feature('v2').set('solnum', 'auto');

model.sol('sol2').feature('v2').set('notsol', 'sol2');

model.sol('sol2').feature('v2').set('notsolnum', 'auto');

model.sol('sol2').feature('v2').set('notsoluse', 'sol3');

model.sol('sol2').feature('v2').feature('mod1_u').set('solvefor', false);

model.sol('sol2').feature('e1').set('solnum', 'auto');

model.sol('sol2').feature('e1').set('control', 'mode');

model.sol('sol2').feature('e1').set('linpsoluse', 'sol3');

model.sol('sol2').feature('e1').set('neigs', '30');

model.sol('sol2').feature('e1').set('linpmethod', 'sol');

model.sol('sol2').feature('e1').set('transform', 'effective_mode_index');

model.sol('sol2').feature('e1').set('linpsol', 'sol2');

model.sol('sol2').feature('e1').set('shift', '3');

model.sol('sol2').feature('e1').set('linpsoluse', 'sol3');

model.sol('sol2').feature('e1').feature('aDef').set('complexfun', true);

model.sol('sol2').runAll;

model.batch('p1').set('err', true);

model.batch('p1').set('plistarr', \{'40e-9 80e-9 100e-9 120e-9 140e-9 160e-9 180e-9 200e-9'\}); model.batch('p1').set('pname', \{'sld1'\});

model.batch('p1').feature('so1').set('param', \{'sld1","4e-8"' '"sld1","8e-8"' '"sld1","1e-7"'

'"sld1","1.2e-7"' '"sld1","1.4e-7"' '"sld1","1.6e-7"' '"sld1","2e-7"'\});

model.batch('p1').feature('so1').set('store', 'on');

model.batch('p1').run;

model.batch('p2').set('err', true);

model.batch('p2').set('plistarr', \{'40e-9 80e-9 100e-9 120e-9 140e-9 160e-9 180e-9 200e-9'\}); model.batch('p2').set('pname', \{'sld1'\});

model.batch('p2').feature('so1').set('param', \{'"sld1","4e-8"' '"sld1","8e-8"' '"sld1","1e-7"'

'"sld1","1.2e-7"' '"sld1","1.4e-7"' '"sld1","1.6e-7"' '"sld1","1.8e-7"' '"sld1","2e-7"'\});

model.batch('p2').feature('so1').set('store', 'on');

model.batch('p2').run;

model.batch('p3').set('err', true);

model.batch('p3').set('plistarr', \{'20e-9 50e-9 75e-9 85e-9 100e-9 125e-9 150e-9 200e-9

250e-9 300e-9 400e-9'\});

model.batch('p3').set('pname', \{'t1'\}); 
model.batch('p3').feature('so1').set('param', \{'"sld1","4e-8"' '"sld1","8e-8"' '"sld1","1e-7"'

'"sld1","1.2e-7"' '"sld1","1.4e-7"' '"sld1","1.6e-7"' '"sld1","1.8e-7"' '"sld1","2e-7"'\});

model.batch('p3').feature('so1').set('store', 'on');

model.batch('p3').run;

model.batch('p4').set('control', 'param');

model.batch('p4').set('err', true);

model.batch('p4').set('plistarr', \{'20e-9 50e-9 75e-9 85e-9 100e-9 125e-9 150e-9 200e-9'\});

model.batch('p4').set('pname', \{'sld1'\});

model.batch('p4').set('control', 'param');

model.batch('p4').feature('so1').set('psol', 'sol4');

model.batch('p4').feature('so1').set('param', \{'"sld1","2e-8"' '"sld1","5e-8"' '"sld1","7.5e-8"'

'"sld1","8.5e-8"' '"sld1","1e-7"' '"sld1","1.25e-7"' '"sld1","1.5e-7"' '"sld1","2e-7"'\});

model.batch('p4').feature('so1').set('seq', 'sol2');

model.batch('p4').attach('std1');

model.batch('p4').run;

model.result.numerical('int1').set('data', 'dset4');

model.result.numerical('int1').set('looplevelindices', $\left\{21^{\prime}\right.$ " $\}$ );

model.result.numerical('int1').set('unit', ' $\mathrm{m}^{\wedge} 3 * \mathrm{~kg}^{\wedge} 2 /\left(\mathrm{s}^{\wedge} 6^{*} \mathrm{~A}^{\wedge} 2\right)$ ');

model.result.numerical('int1').set('looplevelinput', \{'manualindices' 'all' \});

model.result.numerical('int1').set('table', 'tbl1');

model.result.numerical('int1').set('descr', 'd(solid.eX,Y)*abs(emw.Ex)^2');

model.result.numerical('int1').set('expr', 'd(solid.eX,Y)*abs(emw.Ex)^2');

model.result.numerical('int2').set('data', 'dset4');

model.result.numerical('int2').set('looplevelindices', $\left\{21^{\prime}\right.$ " $\}$ );

model.result.numerical('int2').set('unit', ' $\mathrm{m}^{\wedge} 3 * \mathrm{~kg}^{\wedge} 2 /\left(\mathrm{s}^{\wedge} 6^{*} \mathrm{~A}^{\wedge} 2\right)$ ');

model.result.numerical('int2').set('looplevelinput', \{'manualindices' 'all'\});

model.result.numerical('int2').set('descr', 'd(solid.eX,Y)*abs(emw.Ex)^2');

model.result.numerical('int2').set('expr', 'd(solid.eX,Y)*abs(emw.Ex)^2');

model.result.numerical('int3').set('data', 'dset4');

model.result('pg1').set('looplevel', \{'1' '4'\});

model.result('pg1').feature('surf1').set('descr', 'd(solid.eX,Y)');

model.result('pg1').feature('surf1').set('rangecolormax', '12e4');

model.result('pg1').feature('surf1').set('expr', 'd(solid.eX,Y)');

model.result('pg1').feature('surf1').set('rangecoloractive', 'on');

model.result('pg1').feature('surf1').set('rangecolormin', '-12e4');

model.result('pg1').feature('surf1').set('unit', '1/m');

model.result('pg1').feature('surf1').feature('def').active(false);

model.result('pg1').feature('surf1').feature('def').set('scale', '11.494760815810672');

model.result('pg1').feature('surf1').feature('def').set('scaleactive', false);

model.result('pg2').name('Electric Field (emw)');

model.result('pg2').set('data', 'dset4');

model.result('pg2').set('looplevel', \{'21' '4' $\}$ );

model.result('pg2').set('frametype', 'spatial');

model.result('pg2').set('edgecolor', 'white');

model.result('pg2').feature('surf1').set('descr', 'abs(emw.Ex)^2'); 
model.result('pg2').feature('surf1').set('expr', 'abs(emw.Ex) $\left.{ }^{\wedge} 2^{\prime}\right)$;

model.result('pg2').feature('surf1').set('unit', 'm²* $\left.\mathrm{kg}^{\wedge} 2 /\left(\mathrm{s}^{\wedge} 6^{*} \mathrm{~A}^{\wedge} 2\right)^{\prime}\right)$;

model.result('pg3').name('Stress (solid) 1');

model.result('pg3').set('data', 'dset4');

model.result('pg3').set('looplevel', \{'21' '4'\});

model.result('pg3').feature('surf1').set('descr', 'd(solid.eX,Y)*abs(emw.Ex)^2/207');

model.result('pg3').feature('surf1').set('rangecolormax', '6e4');

model.result('pg3').feature('surf1').set('expr', 'd(solid.eX,Y)*abs(emw.Ex)^2/207');

model.result('pg3').feature('surf1').set('rangecoloractive', 'on');

model.result('pg3').feature('surf1').set('rangecolormin', '-6e4');

model.result('pg3').feature('surf1').set('unit', 'm* $\left.\mathrm{kg}^{\wedge} 2 /\left(\mathrm{s}^{\wedge} 6^{*} \mathrm{~A}^{\wedge} 2\right)^{\prime}\right)$;

model.result('pg3').feature('surf1').feature('def').active(false);

model.result('pg3').feature('surf1').feature('def').set('scale', '11.494760815810672');

model.result('pg3').feature('surf1').feature('def').set('scaleactive', false);

out $=$ model; 


\section{References}

[1] D. Shiri, A. Verma, C. R. Selvakumar, and M. P. Anantram, "Reversible modulation of spontaneous emission by strain in silicon nanowires.," Sci. Rep., vol. 2, p. 461, Jan. 2012.

[2] G. T. Reed, G. Mashanovich, F. Y. Gardes, and D. J. Thomson, "Silicon optical modulators," Nat. Photonics, vol. 4, no. 8, pp. 518-526, Jul. 2010.

[3] Y. Vlasov, W. M. J. Green, and F. Xia, "High-throughput silicon nanophotonic wavelength-insensitive switch for on-chip optical networks," Nat. Photonics, vol. 2, no. 4, pp. 242-246, Mar. 2008.

[4] G. T. Reed, An Introduction to Silicon Photonics, vol. 119. 2006, pp. 161-203.

[5] G. T. Reed, G. Z. Mashanovich, F. Y. Gardes, M. Nedeljkovic, Y. Hu, D. J. Thomson, K. Li, P. R. Wilson, S. Chen, and S. S. Hsu, "Recent breakthroughs in carrier depletion based silicon optical modulators," 2013.

[6] A. Liu, R. Jones, L. Liao, and D. Samara-Rubio, "A high-speed silicon optical modulator based on a metal-oxide-semiconductor capacitor," Nature, vol. 427, no. February, pp. 615-618, 2004.

[7] R. S. Jacobsen, K. N. Andersen, P. I. Borel, J. Fage-Pedersen, L. H. Frandsen, O. Hansen, M. Kristensen, A. V Lavrinenko, G. Moulin, H. Ou, C. Peucheret, B. Zsigri, and A. Bjarklev, "Strained silicon as a new electro-optic material," Nature, vol. 441, no. 7090, pp. 199-202, May 2006.

[8] J. Fage-Pedersen, L. H. Frandsen, A. V. Lavrinenko, and P. I. Borel, "A linear electro-optic effect in silicon induced by use of strain," 3rd IEEE Int. Conf. Gr. IV Photonics, 2006., vol. 3, pp. 37-39, 2006.

[9] B. Chmielak, M. Waldow, C. Matheisen, C. Ripperda, J. Bolten, T. Wahlbrink, M. Nagel, F. Merget, and H. Kurz, "Pockels effect based fully integrated, strained silicon electro-optic modulator.," Opt. Express, vol. 19, no. 18, pp. 17212-9, Aug. 2011.

[10] N. K. Hon, K. K. Tsia, D. R. Solli, B. Jalali, and J. B. Khurgin, "Stress-induced $\chi(2)$ in silicon-comparison between theoretical and experimental values," in Group IV Photonics, 2009, pp. 232-234.

[11] M. W. M. Puckett, J. S. T. J. Smalley, M. Abashin, A. Grieco, and Y. Fainman, "Tensor of the second-order nonlinear susceptibility in asymmetrically strained silicon waveguides: analysis and experimental validation," Opt. Lett., vol. 39, no. 6, p. 1693, Mar. 2014. 
[12] A. Aleali, D. Xu, J. H. Schmid, P. Cheben, and W. N. Ye, "Optimization of stressinduced pockels effect in silicon waveguides for optical modulators," in Group IV Photonics, 10th International Conference on, 2013, vol. 3, no. 2, pp. 109-110.

[13] B. Chmielak, C. Matheisen, C. Ripperda, J. Bolten, T. Wahlbrink, M. Waldow, and H. Kurz, "Investigation of local strain distribution and linear electro-optic effect in strained silicon waveguides," Opt. Express, vol. 21, no. 21, p. 25324, Oct. 2013.

[14] M. Cazzanelli, F. Bianco, E. Borga, G. Pucker, M. Ghulinyan, E. Degoli, E. Luppi, S. Ossicini, D. Modotto, S. Wabnitz, R. Pierobon, L. Pavesi, and V. Véniard, "Second harmonic generation in silicon waveguides strained by silicon nitride," Nat. Mater., vol. 11, no. 2, pp. 148-54, Feb. 2012.

[15] C. Schriever, C. Bohley, and R. B. Wehrspohn, "Strain dependence of secondharmonic generation in silicon.," Opt. Lett., vol. 35, no. 3, pp. 273-5, Feb. 2010.

[16] R. W. Boyd, Nonlinear Optics, 3rd ed. 2008.

[17] E. Hecht, "The Kerr and Pockels effects," in Optics, 4th ed., A. Black, Ed. San Francisco: Addison Wesley, 2002, pp. 268-370.

[18] “Centrosymmetry," Wikipedia, 2014. [Online]. Available: http://en.wikipedia.org/wiki/Centrosymmetry. [Accessed: 17-Feb-2014].

[19] D. Guidotti, T. A. Driscoll, and H. J. Gerritsen, "Second harmonic generation in centro-symmetric semiconductors," Solid State Commun., vol. 46, no. 4, pp. 337$340,1983$.

[20] S. Kasap and H. Ruda, An Illustrated Dictionary of Optoelectronics and Photonics : Important Terms and Effects, 2nd ed., vol. 1, no. February. 2002, pp. $1-169$.

[21] P. A. Franken, A. E. Hill, C. W. Peters, and G. Weinreich, "Generation of optical harmonics," Phys. Rev. Lett., vol. 7, no. 4, pp. 118-120, 1961.

[22] S. V. Govorkov, V. I. Emel'yanov, N. I. Koroteev, G. I. Petrov, I. L. Shumay, V. V. Yakovlev, and R. V. Khokhlov, "Inhomogeneous deformation of silicon surface layers probed by second-harmonic generation in reflection," J. Opt. Soc. Am. B, vol. 6, no. 6, p. 1117, Jun. 1989.

[23] C. Schriever, C. Bohley, and R. B. Wehrspohn, Mechanical stress on the nanoscale: simulation, material systems and characterization techniques. Weinheim: Wiley, 2011, pp. 333-355. 
[24] A. Boudrioua, "The Electro-optic effect in waveguides," in Photonic Waveguides: Theory and Applications, Hoboken, NJ, USA: John Wiley \& Sons, Ltd, 2010, pp. 187-259.

[25] B. E. A. Saleh and M. C. Teich, "Principles of Electro-Optics," in Fundamentals of Photonics, 1st ed., New York: John Wiley \& Sons, Inc., 1991, pp. 696-735.

[26] G. Li and P. Yu, "Optical intensity modulators for digital and analog applications," J. Light. Technol., vol. 21, no. 9, pp. 2010-2030, 2003.

[27] E. L. Wooten, K. M. Kissa, A. Yi-Yan, E. J. Murphy, D. A. Lafaw, P. F. Hallemeier, D. Maack, D. V. Attanasio, D. J. Fritz, G. McBrien, and D. E. Bossi, "A review of lithium niobate modulators for fiber-optic communications systems," Sel. Top. Quantum Electron. IEEE J., vol. 6, no. 1, pp. 69-82, 2000.

[28] J. Y. Huang, "Probing inhomogeneous lattice deformation at interface of $\mathrm{Si}(111) / \mathrm{SiO} 2$ by optical second sarmonic reflection and Raman spectroscopy," Jpn. J. Appl. Phys., vol. 33, no. Part 1, No. 7A, pp. 3878-3886, Jul. 1994.

[29] J. Sipe, D. Moss, and H. Van Driel, "Phenomenological theory of optical secondand third-harmonic generation from cubic centrosymmetric crystals," Phys. Rev. B, vol. 35, no. 3, pp. 1129-1141, 1987.

[30] M. Notomi, K. Yamada, A. Shinya, J. Takahashi, C. Takahashi, and I. Yokohama, "Extremely large group-velocity dispersion of line-defect waveguides in photonic crystal slabs," Phys. Rev. Lett., vol. 87, no. 25, p. 253902, Nov. 2001.

[31] M. Maeda and K. Ikeda, "Stress evaluation of radio-frequency-biased plasmaenhanced chemical vapor deposited silicon nitride films," J. Appl. Phys., vol. 83, no. 7, p. 3865, 1998.

[32] W. N. Ye, D.-X. Xu, S. Janz, P. Cheben, M. J. Picard, B. Lamontagne, and N. G. Tarr, "Birefringence control using stress engineering in silicon-on-insulator (SOI) waveguides," J. Light. Technol., vol. 23, no. 3, pp. 1308-1318, 2005.

[33] J. Humlicek, "Silicon-germanium alloys (SixGe1_x) revisited," in Handbook of Optical Constants of Solids, vol. III, E. D. Palik, Ed. San Diego: Academic Press, 1984, pp. 537-552.

[34] W. Martienssen and H. Warlimont, Springer Handbook of Condensed Matter and Materials Data. Heidelberg: Springer Berlin Heidelberg, 2005, p. 1119.

[35] P. J. Burkhardt and R. F. Marvel, "Thermal expansion of sputtered silicon nitride films,” J. Electrochem. Soc., vol. 116, no. 6, p. 864, 1969. 
[36] B. Walmsley, Y. Liu, and X. Hu, "Poisson's ratio of low-temperature PECVD silicon nitride thin films," J. Microelectromechanical Syst., vol. 16, no. 3, pp. 622627, 2007.

[37] E. Cianci, a. Coppa, and V. Foglietti, "Young's modulus and residual stress of DF PECVD silicon nitride for MEMS free-standing membranes," Microelectron. Eng., vol. 84, no. 5-8, pp. 1296-1299, May 2007.

[38] K. Takimoto, a. Fukuta, Y. Yamamoto, N. Yoshida, T. Itoh, and S. Nonomura, "Linear thermal expansion coefficients of amorphous and microcrystalline silicon films," J. Non. Cryst. Solids, vol. 299-302, pp. 314-317, Apr. 2002.

[39] A. E. Franke, J. M. Heck, T. King, S. Member, and R. T. Howe, "Polycrystalline silicon - germanium films for integrated microsystems," vol. 12, no. 2, pp. 160 $171,2003$.

[40] J. J. Wortman and R. a. Evans, "Young's modulus, shear modulus, and Poisson's ratio in silicon and germanium," J. Appl. Phys., vol. 36, no. 1, p. 153, 1965.

[41] E. Cianci, a. Schina, a. Minotti, S. Quaresima, and V. Foglietti, "Dual frequency PECVD silicon nitride for fabrication of CMUTs' membranes," Sensors Actuators A Phys., vol. 127, no. 1, pp. 80-87, Feb. 2006.

[42] G. Xu, P. Jin, M. Tazawa, and K. Yoshimura, "Optical investigation of silicon nitride thin films deposited by r.f. magnetron sputtering," Thin Solid Films, vol. 425, no. 1-2, pp. 196-202, Feb. 2003.

[43] C.-L. Tien and T.-W. Lin, "Thermal expansion coefficient and thermomechanical properties of $\mathrm{SiN}(\mathrm{x})$ thin films prepared by plasma-enhanced chemical vapor deposition.," Appl. Opt., vol. 51, no. 30, pp. 7229-35, Oct. 2012.

[44] F. Bianco, K. Fedus, F. Enrichi, R. Pierobon, M. Cazzanelli, M. Ghulinyan, G. Pucker, and L. Pavesi, "Two-dimensional micro-Raman mapping of stress and strain distributions in strained silicon waveguides," Semicond. Sci. Technol., vol. 27, no. 8, p. 085009, Aug. 2012.

[45] M. Huang, "Stress effects on the performance of optical waveguides," Int. J. Solids Struct., vol. 40, no. 7, pp. 1615-1632, Apr. 2003.

[46] M. Maeda and K. Ikeda, "Stress evaluation of radio-frequency-biased plasmaenhanced chemical vapor deposited silicon nitride films," J. Appl. Phys., vol. 83, no. 7 , p. 3865,1998 .

[47] M. Blech, A. Laades, C. Ronning, B. Schröter, C. Borschel, D. Rzesanke, and A. Lawerenz, "Detailed study of PECVD silicon nitride and correlation of various 
characterization techniques," in 24th European Photovoltaic Solar Energy Conference, 2009, pp. 507-511.

[48] J. Lacey and F. Payne, "Radiation loss from planar waveguides with random wall imperfections," Optoelectron. IEE Proc. J, vol. 137, no. 4, pp. 282-288, 1990.

[49] C. D. Krzeminski, "Stress mapping in strain-engineered silicon p-type MOSFET device: A comparison between process simulation and experiments," J. Vac. Sci. Technol. B Microelectron. Nanom. Struct., vol. 30, no. 2, pp. 022203-022203, 2012.

[50] M. a. Capano, "Residual strains in cubic silicon carbide measured by Raman spectroscopy correlated with x-ray diffraction and transmission electron microscopy," J. Appl. Phys., vol. 100, no. 8, p. 083514, 2006.

[51] E. Johlin, N. Tabet, S. Castro-Galnares, A. Abdallah, M. I. Bertoni, T. Asafa, J. C. Grossman, S. Said, and T. Buonassisi, "Structural origins of intrinsic stress in amorphous silicon thin films," Phys. Rev. B, vol. 85, no. 7, p. 075202, Feb. 2012.

[52] X. Jiang, K. Reichelt, and B. Stritzker, "The hardness and Young's modulus of amorphous hydrogenated carbon and silicon films measured with an ultralow load indenter," J. Appl. Phys., vol. 66, no. 12, p. 5805, 1989.

[53] R. Kuschnereit, H. Fath, A. A. Kolomenskii, M. Szabadi, and P. Hess, "Mechanical and elastic properties of amorphous hydrogenated silicon films studied by broadband surface acoustic wave spectroscopy," Appl. Phys. A, vol. 61, no. 3, pp. 269-276, 1995.

[54] C. R. Miranda, K. V. Tretiakov, and S. Scandolo, "A computational study of elastic properties of disordered systems with voids," J. Non. Cryst. Solids, vol. 352, no. 40-41, pp. 4283-4286, Nov. 2006.

[55] a. . Korhonen, P. . Jones, and F. . Cocks, "On the thermoelastic properties of hydrogenated amorphous silicon,” Mater. Sci. Eng., vol. 49, no. 2, pp. 127-132, Jul. 1981.

[56] R. B. Wehrspohn, S. C. Deane, I. D. French, I. Gale, J. Hewett, M. J. Powell, and J. Robertson, "Relative importance of the $\mathrm{Si}-\mathrm{Si}$ bond and $\mathrm{Si}-\mathrm{H}$ bond for the stability of amorphous silicon thin film transistors," J. Appl. Phys., vol. 87, no. 1, p. 144, 2000.

[57] M. J. Madou, "Molecular Beam Epitaxy," in Fundamentals of Microfabrication, The Sience of Miniaturaization, 2nd ed., M. J. Madou, Ed. Boca Raton: CRC Press, 2001, pp. 140-141. 
[58] D.-X. Xu, "Polarization control in silicon photonic waveguide components using cladding stress engineering," in Silicon Photonics II, Components and Integration, vol. 119, D. J. Lockwood and L. Pavesi, Eds. Berlin, Heidelberg: Springer, 2011, pp. $31-70$.

[59] P. Ashburn and D. Bagnall, "Silicon-germanium: properties, growth and applications," in Springer Handbook of Electronic and Photonic Materials, P. Ashburn and D. Bagnall, Eds. Springer, 2007, pp. 481-498. 